\title{
\#USES
}

science for a changing world

Prepared in cooperation with the Colorado Department of Transportation

\section{Paleoflood Investigations to Improve Peak-Streamflow Regional-Regression Equations for Natural Streamflow in Eastern Colorado, 2015}
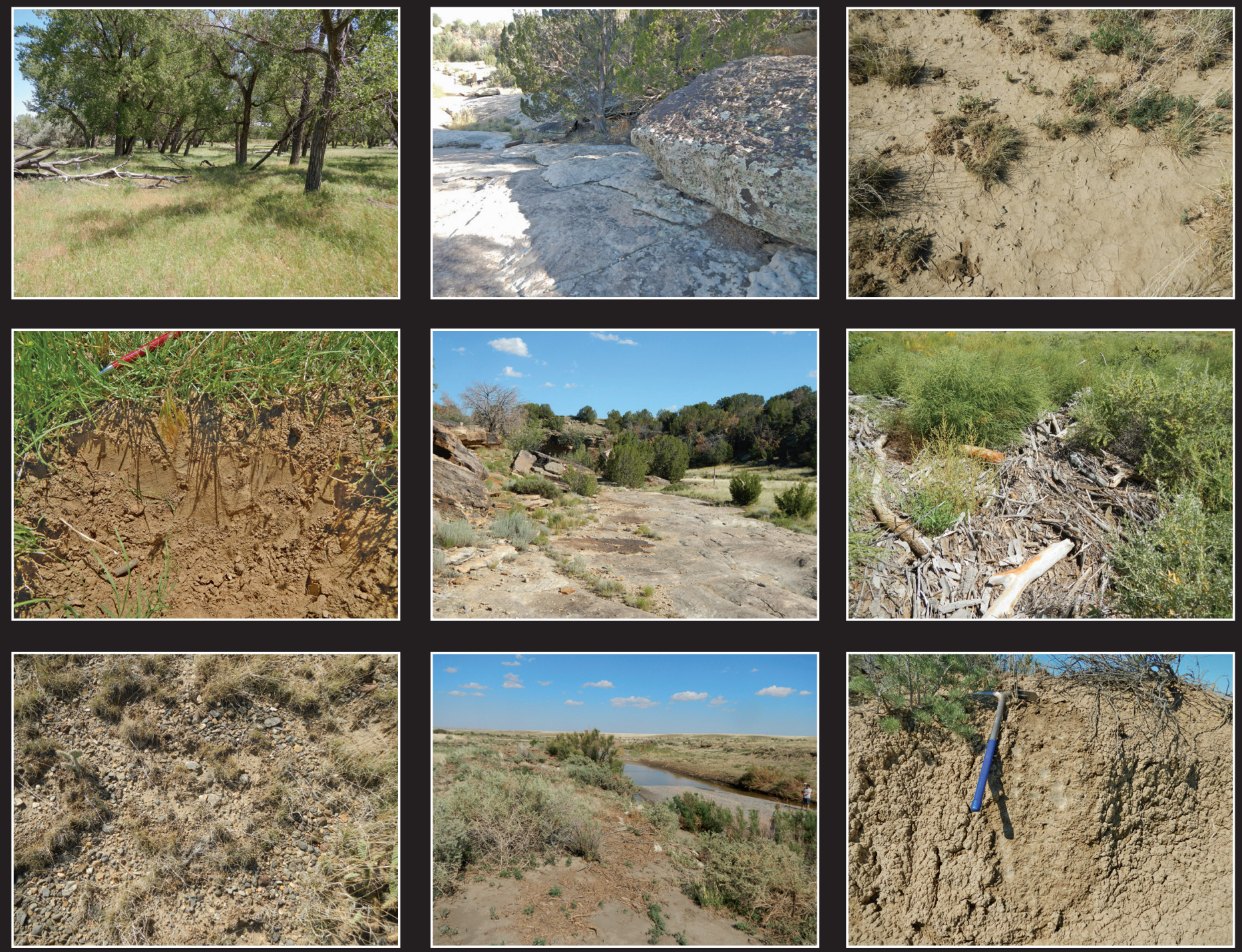

Scientific Investigations Report 2016-5099

U.S. Department of the Interior

U.S. Geological Survey 

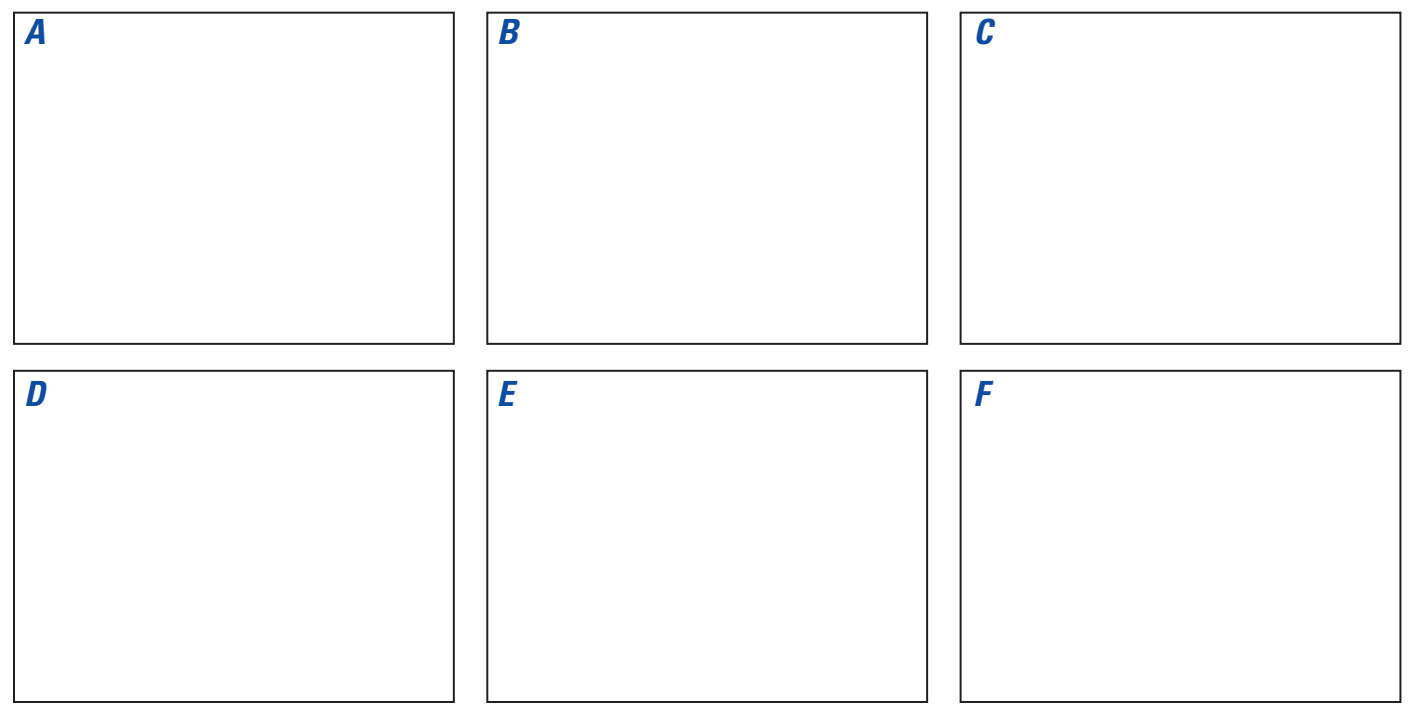

G
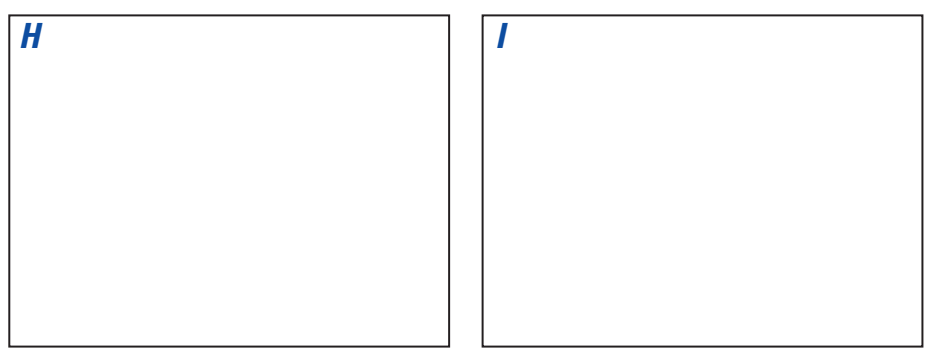

Cover. A, Arikaree River at Haigler, Nebr.; $B$, Taylor Arroyo below Rock Crossing near Thatcher, Colo.; C, Frijole Creek near Alfalfa, Colo.; D, Arikaree River at Haigler, Nebr.; $E$, Taylor Arroyo below Rock Crossing near Thatcher, Colo.; F, Little Fountain Creek near Fountain, Colo.; G, Frijole Creek near Alfalfa, Colo.; $H$, Frijole Creek near Alfalfa, Colo.; I, Big Arroyo near Thatcher, Colo. All cover photographs by Tessa Harden, USGS. 


\section{Paleoflood Investigations to Improve Peak-Streamflow Regional-Regression Equations for Natural Streamflow in Eastern Colorado, 2015}

By Michael S. Kohn, Michael R. Stevens, Tessa M. Harden, Jeanne E. Godaire, Ralph E. Klinger, and Amanullah Mommandi

Prepared in cooperation with the Colorado Department of Transportation

Scientific Investigations Report 2016-5099 


\title{
U.S. Department of the Interior SALLY JEWELL, Secretary
}

\section{U.S. Geological Survey Suzette M. Kimball, Director}

\author{
U.S. Geological Survey, Reston, Virginia: 2016
}

For more information on the USGS - the Federal source for science about the Earth, its natural and living resources, natural hazards, and the environment—visit http://www.usgs.gov or call 1-888-ASK-USGS.

For an overview of USGS information products, including maps, imagery, and publications, visit http://store.usgs.gov/.

Any use of trade, firm, or product names is for descriptive purposes only and does not imply endorsement by the U.S. Government.

Although this information product, for the most part, is in the public domain, it also may contain copyrighted materials as noted in the text. Permission to reproduce copyrighted items must be secured from the copyright owner.

Suggested citation:

Kohn, M.S., Stevens, M.R., Harden, T.M., Godaire, J.E., Klinger, R.E., and Mommandi, Amanullah, 2016, Paleoflood investigations to improve peak-streamflow regional-regression equations for natural streamflow in eastern Colorado, 2015: U.S. Geological Survey Scientific Investigations Report 2016-5099, 58 p., http://dx.doi.org/10.3133/sir20165099.

ISSN 2328-0328 (online) 


\section{Acknowledgments}

The authors would like to recognize Robert Jarrett, formerly of the U.S. Geological Survey (USGS), who was instrumental in helping develop the concept for this study and a plan to execute it. Julie Kiang of the USGS offered invaluable technical assistance during the undertaking of the study. Andrea Veilleux of the USGS provided support and guidance throughout the study and as a technical reviewer. John Fulton and Jim O'Connor of the USGS contributed constructive comments as technical reviewers, which enhanced the quality of the analysis and report. Phil DeArcos and Doug Stenzel of the Colorado Division of Water Resources compiled peak-streamflow data collected by the Colorado Division of Water Resources used in this study. Ben Dietsch of the USGS compiled peak-streamflow data collected by the Nebraska Department of Natural Resources used in this study. Colorado Division of Water Resources lead hydrographers Russell Stroud (Region 1) and Joseph Talbott (Region 2) provided assistance accessing Colorado Division of Water Resources streamgages. Timothy Cohn of the USGS wrote computer program code to compute the effective record length at sites where paleoflood data were available. Andy Bock of the USGS wrote computer program code that creates input files for the peak-streamflow regional-regression equation program. Steve Char of the USGS computed the basin and climate characteristics using a geographic information system. 



\section{Contents}

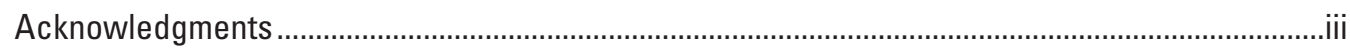

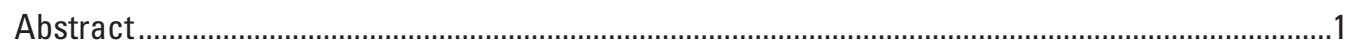

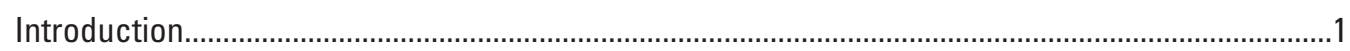

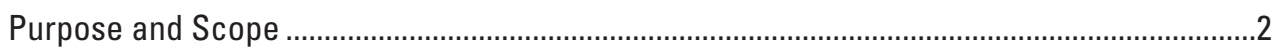

Description of the Study Area ..........................................................................................

Previous Studies and Background Information.....................................................................

Methods for Data Development for Streamgages ……..........................................................

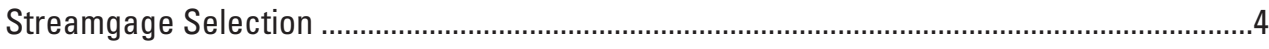

Paleoflood Investigations .......................................................................................................

Paleoflood Slackwater Deposits ...................................................................................12

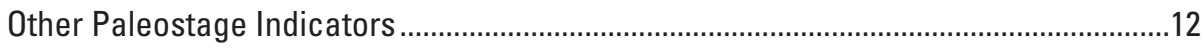

Methodology Used to Identify Paleofloods and Respective Ages ...................................13

Methodology Used to Identify Non-Exceedance Bounds and Respective Ages ...........13

Computing Discharge of Paleofloods ............................................................................17

Hydrologic Engineering Center River Analysis System Computation.....................17

Slope-Area Computation ....................................................................................17

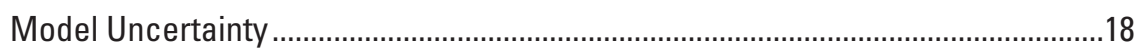

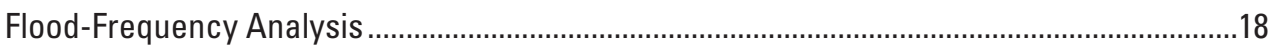

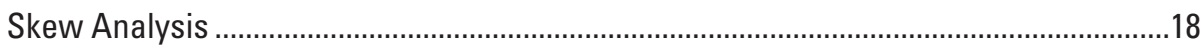

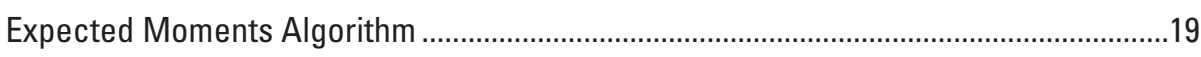

Multiple Grubbs-Beck Test for Detecting Potentially Influential Low Floods..................19

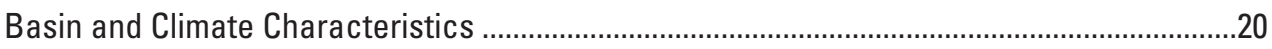

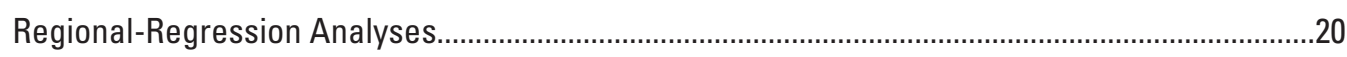

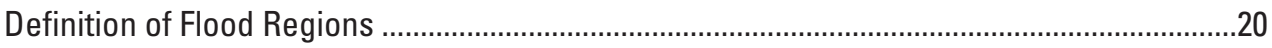

Development of Peak-Streamflow Regional-Regression Equations.....................................22

Ordinary-Least Squares Regression ......................................................................24

Generalized-Least Squares Regression ..................................................................24

Final Peak-Streamflow Regional-Regression Equations ....................................................2

Application and Limitations of Peak-Streamflow Regional-Regression Equations.................28

Use of Peak-Streamflow Regional-Regression Equations at Streamgages ...................28

Use of Peak-Streamflow Regional-Regression Equations on Gaged Streams ..............29

Use of Peak-Streamflow Regional-Regression Equations on Ungaged Streams...........30

Use of Peak-Streamflow Regional-Regression Equations on Ungaged Streams

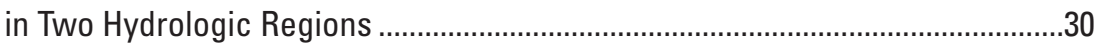

Effect of Paleoflood Data on the Peak-Streamflow Regional-Regression Equations.............30

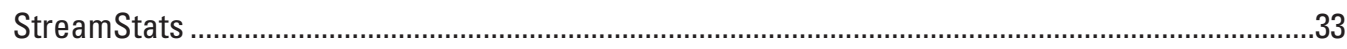

Summary

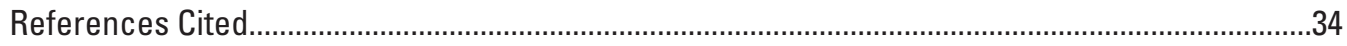


Appendixes

1. Example paleoflood investigation and computation of streamgage peak-streamflow frequency at 06758700 Middle Bijou Creek tributary near Deer Trail, Colo.........................43

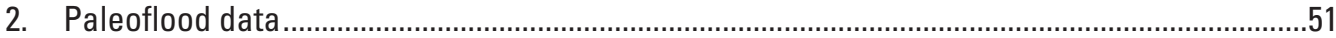

3. Annual exceedance-probability discharges ………............................................................

4. Flood-frequency analysis at the 188 streamgages included in report .................................. link

5. Variance of prediction values and annual exceedance-probability discharges for streamgages in the Foothills and Plains hydrologic regions of Colorado and selected streamgages in adjacent states posed. link

6. Final peak-streamflow regional-regression equation analyses in the Foothills and Plains hydrologic regions of Colorado link

\section{Figures}

1. Map showing boundaries of the hydrologic regions from the previous flood-frequency study in Colorado

2. Map showing location of the 188 streamgages used to develop the peakstreamflow regional-regression equations, of which 44 streamgages have paleoflood or non-exceedance bound data

3. Graphs showing flood-frequency analyses for 07125100 Frijole Creek near Alfalfa, Colo., using the $A, 13$-year streamgage record only and $B$, streamgage record with one paleoflood and one non-exceedance bound; the floodfrequency curve and its 95 -percent confidence limits are reduced with the addition of the paleoflood data; where threshold is the years for which the historic or paleoflood data is applicable.

4. Idealized channel cross section illustrating the concept of a non-exceedance bound and the fluvial landforms and related deposits relative to paleoflood studies

5.-7. Photographs showing:

5. Evidence of flooding at $A, 07105940$ Little Fountain Creek near Fountain, Colo., B, 07125100 Frijole Creek near Alfalfa, Colo., C, 06821500 Arikaree River at Haigler, Nebr., and D, 07103960 Kettle Creek above U.S. Air Force Academy, Colo.

6. Evidence used to determine non-exceedance bounds. $A$, trimline at 07126390 Lockwood Canyon Creek near Thatcher, Colo., B, well developed, stable soil at 07120620 Big Arroyo near Thatcher, Colo., $C$, weak desert pavement at 07125100 Frijole Creek near Alfalfa, Colo., D, also at 07125100 Frijole Creek near Alfalfa, Colo., no desert pavement development indicating a recently disturbed surface

7. Evidence used to determine non-exceedance bounds. $A$, freshly scoured rock and weathered rock at 07126325 Taylor Arroyo below Rock Crossing, near Thatcher, Colo., $B$, lichens that have not been scoured from recent floods and freshly scoured rock at 07126325 Taylor Arroyo below Rock Crossing, near Thatcher, Colo., $C$, similar aged stand of cottonwood trees at 06821500 Arikaree River at Haigler, Nebr., D, old cottonwood at 06687000 Blue Creek near Lewellen, Nebr.

8. Map showing boundaries of the new hydrologic regions created in eastern Colorado: the Plains hydrologic region and the Foothills hydrologic region with the location of the 99 and 89 streamgages were used to develop the peakstreamflow regional-regression equations in the Plains and Foothills hydrologic regions, respectively

9. Peak-streamflow regional-regression equations for the Foothills hydrologic region ........25

10. Peak-streamflow regional-regression equations for the Plains hydrologic region ............26 
11. Graphs showing relation between 1-percent annual exceedance-probability discharges computed from at-site streamflow to those predicted from the peak-streamflow regional-regression equations for the Foothills and Plains hydrologic regions of Colorado....

12. Graph showing relation between 1-percent annual exceedance-probability discharges computed from the peak-streamflow regional-regression equations in the Foothills and Plains hydrologic regions of Colorado and the 1-percent annual exceedance-probability discharges computed from the peak-streamflow regional-regression equations in Capesius and Stephens (2009)

\section{Appendix Figures}

1-1. Photograph looking downstream toward the discontinued crest-stage gage at 06758700 Middle Bijou Creek Tributary near Deer Trail, Colo.; paleoflood evidence was identified in a terrace along the right bank approximately 100 feet downstream from the streamgage.

1-2. Photograph looking downstream at 06758700 Middle Bijou Creek Tributary near Deer Trail, Colo.; paleoflood evidence was identified in a terrace along the right bank approximately 100 feet downstream from the streamgage; photograph taken from the discontinued crest-stage gage ..

1-3. Photograph showing four horizons are present in this terrace exposure; $A$, an organic rich $A$ horizon, $B$, a $B$ horizon with sand and silt interbeds, $C$, a more developed $B_{2}$ horizon, and $D$, and older $B k$ horizon at the bottom

1-4. Photograph showing these soil samples correspond to the soil horizons in figure 1-3. The soil structure goes from single grained and weakly granular in the A horizon to weak to moderate subangular blocky in the Bk horizon

1-5-1-8. Graphs showing:

1-5. Lower limit discharge model output from Slope-Area Computation Graphical User Interface at the cross section where the terrace with paleoflood evidence was located; the lower limit discharge of 1,290 cubic feet per second ( $\mathrm{ft}^{3} / \mathrm{s}$ or $\mathrm{cfs}$ ) was computed by using the lowest stage of the paleoflood evidence, increasing the Manning roughness coefficient (n) by 20 percent, and decreasing the channel slope by 25 percent

1-6. The upper limit discharge model output from Slope-Area Computation Graphical User Interface at the cross section where the terrace with paleoflood evidence was located; the upper limit discharge of 4,540 cubic feet per second ( $\mathrm{ft}^{3} / \mathrm{s}$ or $\mathrm{cfs}$ ) was computed by using the highest stage of the paleoflood evidence plus 1 foot 3 feet for nonexceedance bounds), decreasing the Manning roughness coefficient (n) by 20 percent, and increasing the channel slope by 25 percent

1-7. The flood-frequency curve for 06758700 Middle Bijou Creek Tributary near Deer Trail, Colo. prior to the inclusion of any paleoflood data; the 0.01 annual exceedance-probability discharge is 2,620 cubic feet per second $\left(\mathrm{ft}^{3} / \mathrm{s}\right)$ and the 95 percent confidence intervals are $1,180 \mathrm{ft}^{3} / \mathrm{s}$ and $29,900 \mathrm{ft}^{3} / \mathrm{s}$

1-8. The flood-frequency curve for 06758700 Middle Bijou Creek Tributary near Deer Trail, Colo. with a paleoflood that has a lower limit discharge of 1,290 cubic feet per second ( $\left.\mathrm{ft}^{3} / \mathrm{s}\right)$, upper limit discharge of $4,540 \mathrm{ft}^{3} / \mathrm{s}$, and age of 150 years; the 0.01 annual exceedance-probability discharge has been reduced by 41 percent to 1,540 $\mathrm{ft}^{3} / \mathrm{s}$ and the 95 percent confidence intervals are $999 \mathrm{ft}^{3} / \mathrm{s}$ and $2,780 \mathrm{ft}^{3} / \mathrm{s}$. 


\section{Tables}

1. Summary of the streamgages used in the regression analysis of natural streams in eastern Colorado, 2015

2. Basin and climate characteristics evaluated for use in the peak-streamflow regional-regression equations in eastern Colorado, 2015.

3. Standard error of prediction of the peak-streamflow regional-regression equations for eastern Colorado presented in the previous study, Foothills hydrologic region, and Plains hydrologic region.

4. Regional exponents and constants determined from regional regression of log-transformed drainage area for area-weighting method to estimate annual exceedance-probability discharges for ungaged sites on gaged streams

5. Number of years of record at the 44 streamgages where paleoflood data were collected

6. Record length of the 188 streamgages by region used in the analysis by hydrologic region and the effect of paleoflood data on the record length for various annual exceedance-probability discharges

7. Standard error of prediction of the peak-streamflow regional-regression equations for eastern Colorado across both hydrologic regions for the 44 streamgages where paleoflood data were collected with and without the inclusion of the paleoflood data.

8. Standard error of prediction of the peak-streamflow regional-regression equations for eastern Colorado with and without the inclusion of the paleoflood data

\section{Appendix Tables}

2-1. Summary of the paleoflood data collected and applied at 44 streamgages used in the regression analysis of natural streams in eastern Colorado, 2015.

$3-1$. Summary of the annual exceedance-probability discharges at the 188 streamgages used in the regression analysis of natural streams in eastern Colorado, 2015.

\section{Conversion Factors}

Inch/Pound to International System of Units

\begin{tabular}{|c|c|c|}
\hline Multiply & By & To obtain \\
\hline \multicolumn{3}{|c|}{ Length } \\
\hline inch (in.) & 2.54 & centimeter $(\mathrm{cm})$ \\
\hline inch (in.) & 25.4 & millimeter (mm) \\
\hline foot $(\mathrm{ft})$ & 0.3048 & $\operatorname{meter}(\mathrm{m})$ \\
\hline mile (mi) & 1.609 & kilometer $(\mathrm{km})$ \\
\hline \multicolumn{3}{|c|}{ Area } \\
\hline square mile $\left(\mathrm{mi}^{2}\right)$ & 259.0 & hectare (ha) \\
\hline square mile $\left(\mathrm{mi}^{2}\right)$ & 2.590 & square kilometer $\left(\mathrm{km}^{2}\right)$ \\
\hline \multicolumn{3}{|c|}{ Volume } \\
\hline cubic foot $\left(\mathrm{ft}^{3}\right)$ & 28.32 & cubic decimeter $\left(\mathrm{dm}^{3}\right)$ \\
\hline cubic foot $\left(\mathrm{ft}^{3}\right)$ & 0.02832 & cubic meter $\left(\mathrm{m}^{3}\right)$ \\
\hline \multicolumn{3}{|c|}{ Flow rate } \\
\hline cubic foot per second $\left(\mathrm{ft}^{3} / \mathrm{s}\right)$ & 0.02832 & cubic meter per second $\left(\mathrm{m}^{3} / \mathrm{s}\right)$ \\
\hline
\end{tabular}


Vertical coordinate information is referenced to the North American Vertical Datum of 1988

(NAVD 88).

Horizontal coordinate information is referenced to the North American Datum of 1983 (NAD 83).

Elevation, as used in this report, refers to distance above the vertical datum.

Water year in this report is defined as the period from 0ctober 1st of one year through

September 30th of the following year and is named for the year of the ending date.

\section{Abbreviations and Acronyms}

$\begin{array}{ll}\text { adjR } & \text { adjusted coefficient of determination } \\ \text { AEP } & \text { annual exceedance-probability } \\ \text { AEPD } & \text { annual exceedance-probability discharge } \\ \text { CDWR } & \text { Colorado Division of Water Resources } \\ \text { DAR } & \text { drainage-area ratio } \\ \text { EMA } & \text { Expected Moments Algorithm } \\ \text { GLS } & \text { Generalized-Least Squares } \\ \text { HEC-RAS } & \text { Hydrologic Engineering Center River Analysis System } \\ \text { Mallow's } C p & \text { measure of the total squared error for a subset model containing the number } \\ \text { MGB } & \text { (n) of independent variables } \\ \text { NDNR } & \text { multiple Grubbs-Beck test } \\ \text { NWIS } & \text { Nebraska Department of Natural Resources } \\ \text { OLS } & \text { National Water Information System } \\ \text { PILF } & \text { Ordinary-Least Squares } \\ \text { PRESS } & \text { Potentially Influential Low Flood } \\ \text { pseudo } R^{2} & \text { Predicted Residual Sum of Squares } \\ \text { PSRRE } & \text { pseudo coefficient of determination } \\ \text { R } & \text { peak-streamflow regional-regression equations } \\ \text { SACGUI } & \text { coefficient of determination } \\ \text { SEP } & \text { slope-area computation graphical user interface } \\ \text { SME } & \text { standard error of prediction } \\ \text { USGS } & \text { standard model error } \\ \text { WLS } & \text { U.S. Geological Survey } \\ \text { Weighted-Least Squares }\end{array}$





\title{
Paleoflood Investigations to Improve Peak-Streamflow Regional-Regression Equations for Natural Streamflow in Eastern Colorado, 2015
}

\author{
By Michael S. Kohn, ${ }^{1}$ Michael R. Stevens, ${ }^{1}$ Tessa M. Harden, ${ }^{2}$ Jeanne E. Godaire, ${ }^{3}$ Ralph E. Klinger ${ }^{3}$ \\ and Amanullah Mommandi ${ }^{4}$
}

\section{Abstract}

The U.S. Geological Survey (USGS), in cooperation with the Colorado Department of Transportation, developed regionalregression equations for estimating the 50-, 20-, 10-, 4-, 2-, 1-, 0.5-, 0.2-percent annual exceedance-probability discharge (AEPD) for natural streamflow in eastern Colorado. A total of 188 streamgages, consisting of 6,536 years of record and a mean of approximately 35 years of record per streamgage, were used to develop the peak-streamflow regional-regression equations. The estimated AEPDs for each streamgage were computed using the USGS software program PeakFQ. The AEPDs were determined using systematic data through water year 2013. Based on previous studies conducted in Colorado and neighboring States and on the availability of data, 72 characteristics (57 basin and 15 climatic characteristics) were evaluated as candidate explanatory variables in the regression analysis. Paleoflood and non-exceedance bound ages were established based on reconnaissance-level methods. Multiple lines of evidence were used at each streamgage to arrive at a conclusion (age estimate) to add a higher degree of certainty to reconnaissance-level estimates. Paleoflood or nonexceedance bound evidence was documented at 41 streamgages, and 3 streamgages had previously collected paleoflood data. To determine the peak discharge of a paleoflood or non-exceedance bound, two different hydraulic models were used.

The mean standard error of prediction (SEP) for all 8 AEPDs was reduced approximately 25 percent compared to the previous flood-frequency study. For paleoflood data to be effective in reducing the $S E P$ in eastern Colorado, a larger ratio than 44 of 188 ( 23 percent) streamgages would need paleoflood data and that paleoflood data would need to increase the record length by more than 25 years for the 1-percent AEPD. The greatest reduction in $S E P$ for the peak-streamflow regional-regression equations was observed when additional new basin characteristics were included in the peak-streamflow regional-regression

\footnotetext{
${ }^{1}$ U.S. Geological Survey, Denver, Colo.

${ }^{2}$ U.S. Geological Survey, Portland, Oreg.

${ }^{3}$ Bureau of Reclamation, Denver, Colo.

${ }^{4}$ State of Colorado Department of Transportation, Denver, Colo.
}

equations and when eastern Colorado was divided into two separate hydrologic regions. To make further reductions in the uncertainties of the peak-streamflow regional-regression equations in the Foothills and Plains hydrologic regions, additional streamgages or crest-stage gages are needed to collect peakstreamflow data on natural streams in eastern Colorado.

Generalized-Least Squares regression was used to compute the final peak-streamflow regional-regression equations for peakstreamflow. Dividing eastern Colorado into two new individual regions at $-104^{\circ}$ longitude resulted in peak-streamflow regionalregression equations with the smallest $S E P$. The new hydrologic region located between $-104^{\circ}$ longitude and the Kansas-Nebraska State line will be designated the Plains hydrologic region and the hydrologic region comprising the rest of eastern Colorado located west of the $-104^{\circ}$ longitude and east of the Rocky Mountains and below 7,500 feet in the South Platte River Basin and below 9,000 feet in the Arkansas River Basin will be designated the Foothills hydrologic region.

\section{Introduction}

The U.S. Geological Survey (USGS), in cooperation with the Colorado Department of Transportation (CDOT), developed peak-streamflow regional-regression equations (PSRREs) for estimating the 50-, 20-, 10-, 4-, 2-, 1-, 0.5-, 0.2-percent annual exceedance-probability discharge (AEPD) for natural streamflow in eastern Colorado. The PSRREs in the Plains hydrologic region in eastern Colorado have the largest uncertainty of any hydrologic region in Colorado (Vaill, 1999; Capesius and Stephens, 2009). Reliable peak-streamflow information is critical for the proper design of stream-related infrastructure, such as bridges and dams, and flood-plain inundation maps. At gaged sites, where sufficient long-term streamflow data have been collected, statistics can be obtained from available publications, by an analysis of available data in the USGS National Water Information System (NWIS) database (http://dx.doi.org/10.5066/F7P55KJN), or other sources of flood information. However, estimates also are needed at ungaged sites where no site-specific streamflow data are available. The use of PSRREs with expressions of predictive uncertainty generally represents a reliable and cost-effective means for 
Paleoflood Investigations to Improve Peak-Streamflow Regional-Regression Equations, Eastern Colorado, 2015

estimating peak streamflow at ungaged sites. The PSRREs are a common tool used to estimate streamflow statistics at ungaged sites across the Nation. The PSRREs are based on statistical relations between (1) peak streamflow from systematic, historic, censored, and paleoflood data at streamgages and (2) basin and climatic characteristics, for which data are typically readily available using a geographic information system (GIS).

\section{Purpose and Scope}

The purpose of this report is to present an updated set of PSRREs for estimating AEPDs for use in eastern Colorado. The PSRRE relate AEPDs to drainage basin size, topography, hydrology, and climatology. This report presents two sets of PSRREs to estimate 8 AEPD statistics that have probabilities of $50,20,10,4,2,1,0.5$, and 0.2 percent, which are equivalent to annual flood-frequency recurrence intervals of 2, 5, 10, 25, 50, 100,200 , and 500 years, respectively. Hereafter, in this report, these statistics are denoted as $Q_{50 \%}$ (in percent [\%]), $Q_{20 \%}, Q_{10 \%}$, $Q_{4 \%}, Q_{2 \%}, Q_{1 \%}, Q_{0.5 \%}$, and $Q_{0.2 \%}$, respectively. The procedure to develop PSRREs included Generalized-Least Squares (GLS) multilinear regression based on base-10 logarithmic transformations of all included variables. The PSRREs were developed both with and without paleoflood data to identify the effect paleoflood data have on the uncertainty of the PSRREs.

Annual peak-discharge data from streamgages with a record of at least 10 years were compiled from the USGS (U.S. Geological Survey, 2013b), Colorado Division of Water Resources (CDWR) (Colorado Division of Water Resources, 2013), and Nebraska Department of Natural Resources (NDNR) (Nebraska Department of Natural Resources, 2013) through water year 2013 as well as paleoflood data. A water year is the 12-month period from October 1 through September 30 designated by the calendar year in which it ends.

The limitations and accuracy of the PSRREs are presented in this report. The study area was extended 100 miles outside Colorado for the purpose of PSRRE development because the hydrology is not affected by State boundaries; however, the PSRREs are only applicable in Colorado. Also, it should be noted that the PSRREs presented in this report are only applicable to natural streamflow with drainage areas between 0.26 and 3,560 square miles $\left(\mathrm{mi}^{2}\right)$. To clarify, the PSRREs are based on analysis of peak-streamflow data at streams relatively unaffected by anthropogenic activities such as storage, regulation, and diversion or return streamflows from a municipality or mining operation, or urban development in a basin. Kircher and others (1985) provide the most quantitative description of natural streamflow as streamflow from drainage basins relatively unaffected by urban development or water-management activities such as substantial reservoir storage, streamflow diversions, or return streamflows of previously diverted streamflow. Further, those authors defined natural streamflow as streamflow having less than about 10 percent of the mean-annual streamflow volume at the streamgage affected by anthropogenic activity. The definition by Kircher and others (1985) was used in Capesius and Stephens (2009) and this report.

\section{Description of the Study Area}

Colorado has a diverse landscape and climate and includes the headwaters of the major river basins of the Colorado, North Platte, South Platte, and Arkansas Rivers and the Rio Grande. The physiographic and hydrologic differences are discussed below.

Colorado can be described by three major physiographic provinces, which trend north to south across the State (Fenneman, 1931). The Great Plains Province, in the eastern 40 percent of the State, consists mostly of grasslands with scattered hills, bluffs, shallow river valleys, and some cultivated areas. The Southern Rocky Mountains Province, west of the Great Plains, includes most of central Colorado from north to south and is characterized by mountain ranges and intermountain valleys. The Colorado Plateau Province is in western Colorado between the Utah State line to the west and the Rocky Mountains to the east. The landscape is distinguished by mesas, plateaus, and eroded canyon terrain that includes much of the western quarter of Colorado from north to south. More detailed descriptions of the major physiographic provinces can be found in Fenneman (1931) and Capesius and Stephens (2009).

The five hydrologic regions of Colorado were defined on the basis of the physiographic and climatic characteristics that were used to develop best-fit PSRREs for previous floodfrequency studies (McCain and Jarrett (1976); Kircher and others, 1985; Vaill, 1999; Capesius and Stephens, 2009) (fig. 1). For this report ".... hydrologic region is qualitatively defined as a region of similar hydrology and climatology. The Mountain hydrologic region is identified as that region of central Colorado above about 7,500 feet in elevation located between the Colorado-Wyoming State line and the Rio Grande Basin. The Mountain hydrologic region encompasses the headwaters of most major river basins in Colorado where the annual peak streamflow generally is produced by snowmelt runoff. The Northwest hydrologic region is defined as the northwestern part of Colorado below 7,500 feet and encompassing substantial areas of the Yampa, White, and Gunnison River Basins. The Rio Grande hydrologic region ranges in elevation from about 5,000 feet near the Colorado-New Mexico State line to more than 14,000 feet in the northern parts and encompasses the Rio Grande Basin. The Southwest hydrologic region is defined as the region located south of the Gunnison River Basin and west of the Rio Grande Basin and encompasses the Dolores and San Juan River Basins. The Plains hydrologic region is east of the Rocky Mountains and below 7,500 feet in the South Platte River Basin and below 9,000 feet in the Arkansas River Basin" (Capesius and Stephens, 2009, p. 4). Because hydrology is not affected by state boundaries, the hydrologic region boundaries were extended 100 miles into all States surrounding Colorado to include more streamgages and improve statistical robustness for development of PSRREs. As a result, the study area includes parts of Kansas, Nebraska, New Mexico, Oklahoma, and Wyoming along with the Plains hydrologic region in Colorado. 


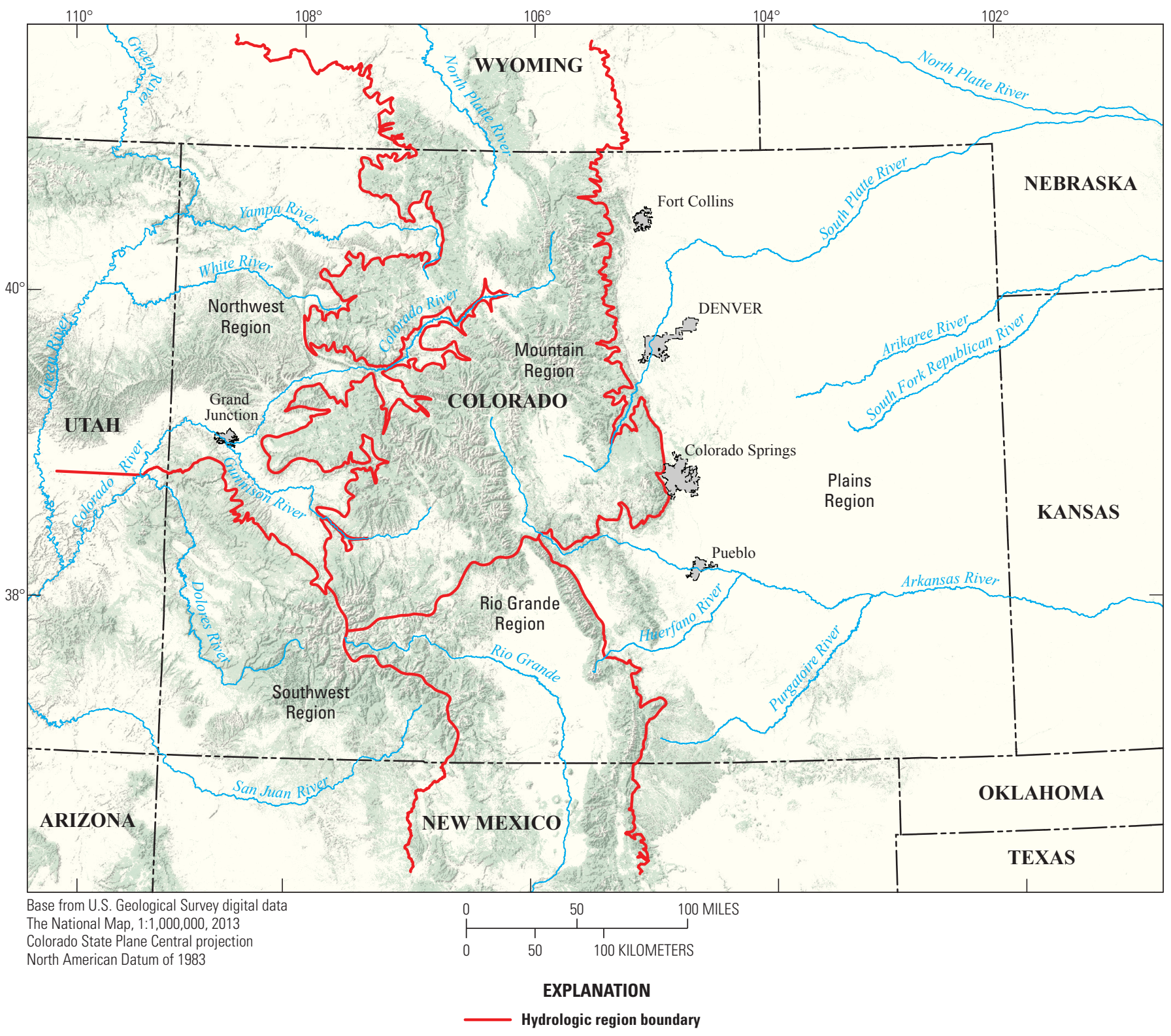

Figure 1. Boundaries of the hydrologic regions from the previous flood-frequency study in Colorado (modified from Capesius and Stephens, 2009).

\section{Previous Studies and Background Information}

Previous studies computed PSRREs in ColoradoPatterson (1964, 1965), Patterson and Somers (1966), Matthai (1968), Hedman and others (1972), McCain and Jarrett (1976), Kircher and others (1985), Livingston and Minges (1987), Vaill (1999), and Capesius and Stephens (2009). Fewer studies have developed regional-regression equations for meanmonthly streamflow, as was done by Kircher and others (1985) and Capesius and Stephens (2009). The hydrologic regions used in this report were delineated by McCain and Jarrett (1976) and were incorporated as the regional framework in Kircher and others (1985) and Capesius and Stephens (2009). Kircher and others (1985) developed regional-regression equations for mean-monthly streamflow in western Colorado for data collected through 1983. Capesius and Stephens (2009) published Statewide peak and non-peak (with the exception of the Plains hydrologic region) statistics (including meanmonthly streamflow) using USGS streamflow data from the beginning of the period of record at each streamgage through water years 2006 and 2007, respectively. In Capesius and Stephens (2009), the PSRREs were developed using USGS systematic and historic data through water year 2006, and the error associated with the PSRREs was characterized using the standard error of prediction (SEP, in percent), the pseudocoefficient of determination (pseudo $R^{2}$, dimensionless), and the standard model error (SME, in percent). Systematic peaks are observed during the collection of the systematic streamgage record, and historic streamflow peaks are observed outside the streamgage record. 
The SEP describes the sum of the model error and sampling error. The SEP is the square root of the mean GLS variance of prediction (Tasker and Stedinger, 1989; Eng and others, 2009). The pseudo $R^{2}$ value is a measure of the percentage of the variation explained by the basin characteristics (explanatory variables) included in the model and is calculated on the basis of the degrees of freedom in the regression (Eash and others, 2013). Griffis and Stedinger (2007) describe how the pseudo $R^{2}$ is more appropriate than the traditional $R^{2}$ or $\operatorname{adj} R^{2}$ in measuring the true variation explained by the explanatory variables in the GLS model. SME measures only the error of the model and does not include sampling error regression (Eash and others, 2013). SME is the square root of the regression model error variance (Tasker and Stedinger, 1989).

\section{Methods for Data Development for Streamgages}

The development of PSRREs in eastern Colorado consists of five steps:

1. Selection of streamgages with natural streamflow conditions for inclusion in multilinear regression analysis,

2. Inclusion of paleoflood investigations conducted at a select number of streamgages where paleoflood evidence exists to provide additional streamflow data to supplement systematic data,

3. Flood-frequency analysis to compute AEPDs for all streamgages using systematic, historic, censored, and paleoflood data if available,

4. Determination of basin and climate characteristics for all the streamgages, and

5. Development of the PSRREs for eastern Colorado.

These steps are further described in the sections that follow.

\section{Streamgage Selection}

The selection of streamgages used for this report was effected by those streamgages selected by Kircher and others (1985), Vaill (1999), Capesius and Stephens (2009), and the authors' knowledge of hydrologic systems in Colorado. A comprehensive list of all USGS streamgages in the Plains hydrologic region and within 100 miles of the Colorado State line adjacent to the Plains hydrologic region was acquired from the NWIS Mapper (USGS, 2013a). From the comprehensive list of candidate streamgages, those streamgages with at least 10 years of streamflow record identified as representative of natural streamflow conditions were selected for this study.

Subsequently, multiple streamgages on the same stream course were evaluated for data independence as judged by the drainage-area ratio (DAR) and the proximity of the basin centroids. Standardized distance is a measure of the normalized, or unit-less distance, between the centroids of two basins and
DAR is used to determine if the size of two basins, when one basin is contained in the other, is sufficiently different that the precipitation events that generate the annual maximum floods in each basin are likely to be different (Veilleux, 2009). Additional information on DAR and basin centroid proximity is found in Asquith and others (2006) and Veilleux (2009). If the DAR was less than 5.0 and the standardized distance was less than 0.5 , the streamgages were determined to be redundant (Veilleux, 2009; Gotvald and others, 2012; Eash and others, 2013; Southard and Veilleux, 2014). In such cases, the streamgage with the longer record was selected. Excluding redundant streamgages on the basis of relative DAR and basin centroid location helps to ensure the independence of the streamflow information among streamgages. This exclusion process serves the purpose of removing redundant data or hydrologic information from the analysis. At the completion of the selection process, 37 streamgages were excluded from analysis because of redundancy, and a total of 188 streamgages, consisting of 6,536 years of record and a mean of approximately 35 years of record per streamgage, were used to develop the PSRREs. A map showing the location of the streamgages is provided in figure 2, and each of the 188 streamgages, ancillary information, and basin and climatic characteristics are listed in table 1 . The "home page" of each USGS streamgage, which provides authoritative streamgage names and other identifying characteristics, can be accessed online at http://waterdata.usgs.gov/ nwis/nwisman/?site_no=STREAMGAGE\&agency_cd=USGS, where the word STREAMGAGE is replaced by the eight-digit USGS streamgage number from table 1.

\section{Paleoflood Investigations}

Most conventional estimates for the frequency of large floods are based on extrapolations from streamgage records, commonly with record lengths shorter than 100 years (Harden and others, 2011). However, this can lead to great uncertainty in AEPDs of 1 percent or less (100-year flood discharge or larger) (fig. 3A) (Blainey and others, 2002; Harden and others, 2011; O'Connor and others, 2014). If a streamgage record contains an exceptionally large flood in the systematic record, the floodfrequency analysis can assign that flood an unrealistically short return period. This is especially true for streamgages with short records. Therefore, estimates of floods with a return period greater than about twice the record can incorporate large uncertainties (fig. 3A) (Harden and others, 2011; O'Connor and others, 2014). Of the 41 streamgages where new paleoflood data were collected in this study, all had streamgage records of less than 100 years and 23 streamgages had records of less than 25 years. The addition of paleoflood data to conventional streamgage data have been shown to increase the accuracy in discharge uncertainty estimates of low probability floods (fig. 3B) (Stedinger and Cohn, 1986; Blainey and others, 2002; Hosman and others, 2003). Even when the paleoflood age was not precisely known (Hosking and Wallis, 1986), the uncertainty estimates of low probability floods were reduced by up to 99 percent (Harden and others 2011; O'Connor and others, 2014). 


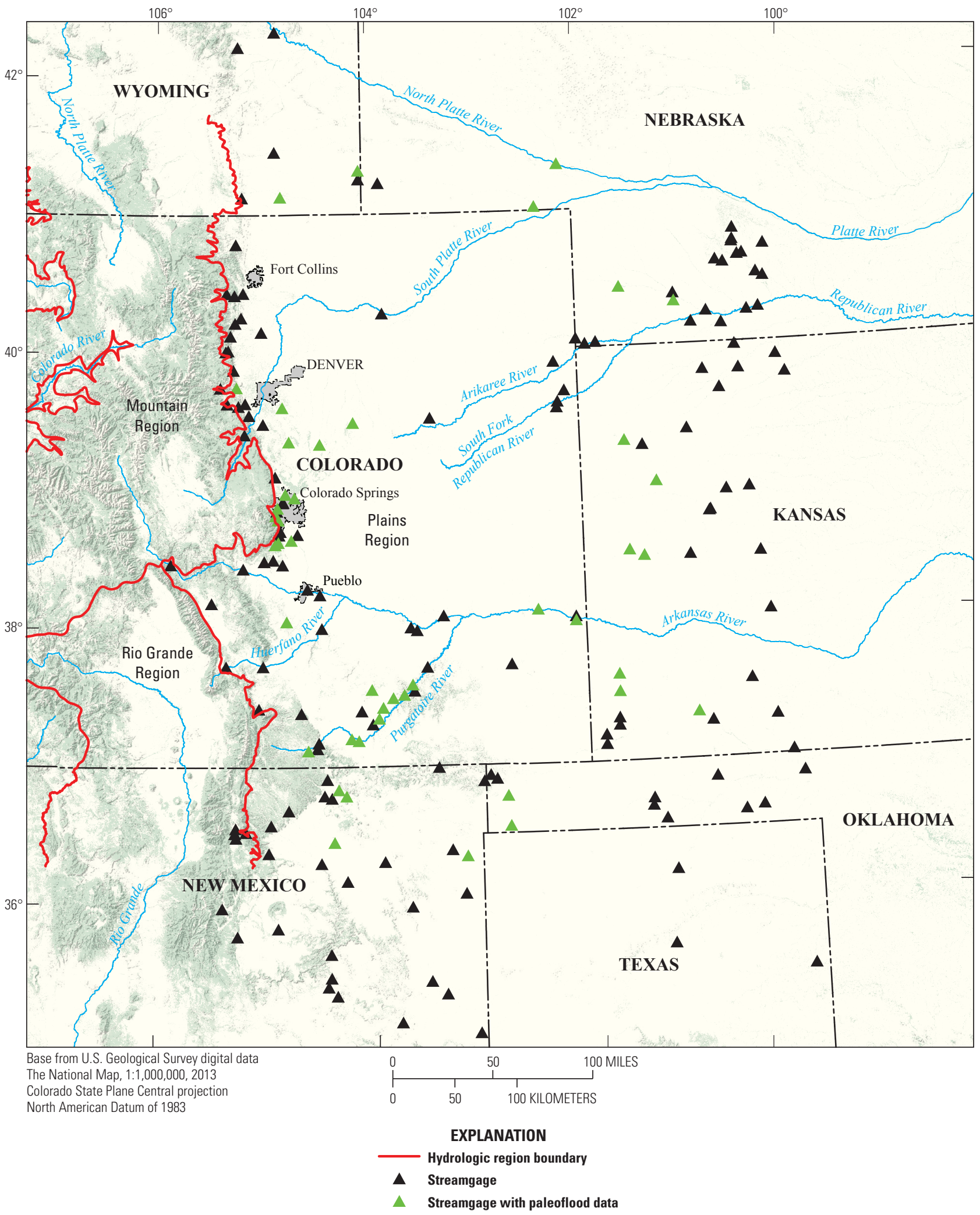

Figure 2. Location of the 188 streamgages used to develop the peak-streamflow regional-regression equations, of which 44 streamgages have paleoflood or non-exceedance bound data. 
Table 1. Summary of the streamgages used in the regression analysis of natural streams in eastern Colorado, 2015.

[USGS, U.S. Geological Survey; CDWR, Colorado Division of Water Resources; NDNR, Nebraska Department of Natural Resources; A, drainage area; mi² , square miles; C, amount of soil consisting of clay in basin; \%, percent; $E_{o w}$, elevation of basin outlet; ft, feet; $P_{100}$, mean basin 6-hour, 100-year precipitation; in., inches; S, mean basin slope; Wyo., Wyoming; Nebr., Nebraska; Colo., Colorado; Kans., Kansas; Okla., Oklahoma; N. Mex., New Mexico; Tex., Texas; NA, not applicable]

\begin{tabular}{|c|c|c|c|c|c|c|c|c|c|c|c|c|}
\hline $\begin{array}{c}\text { USGS } \\
\text { streamgage } \\
\text { number }\end{array}$ & Streamgage name & $\begin{array}{l}\text { Latitude } \\
\text { (decimal } \\
\text { degrees) }\end{array}$ & $\begin{array}{l}\text { Longitude } \\
\text { (decimal } \\
\text { degrees) }\end{array}$ & $\begin{array}{l}\text { USGS } \\
\text { peaks }\end{array}$ & $\begin{array}{l}\text { CDWR } \\
\text { peaks }\end{array}$ & $\begin{array}{l}\text { NDNR } \\
\text { peaks }\end{array}$ & $\begin{array}{l}\text { Paleo- } \\
\text { flood } \\
\text { data }\end{array}$ & $\underset{\left(\mathrm{mi}^{2}\right)}{\mathbf{A}}$ & $\begin{array}{c}\text { C } \\
(\%)\end{array}$ & $\begin{array}{l}E_{\text {out }} \\
\text { (ft) }\end{array}$ & $\begin{array}{l}{ }_{6} \boldsymbol{P}_{100} \\
\text { (in.) }\end{array}$ & $\underset{(\%)}{\mathbf{S}}$ \\
\hline 06655000 & Cottonwood Creek at Wendover, Wyo. & 42.3258 & -104.8764 & 24 & NA & NA & No & 1,960 & 17.3 & 4,450 & 2.59 & 13.6 \\
\hline 06668040 & Rabbit Creek near Wheatland, Wyo. & 42.2158 & -105.2291 & 20 & NA & NA & No & 1.30 & 20.6 & 5,650 & 2.45 & 14.9 \\
\hline 06675300 & Horse Creek Tributary near Little Bear, Wyo. & 41.4558 & -104.8839 & 21 & NA & NA & No & 8.16 & 12.2 & 6,240 & 2.81 & 7.78 \\
\hline 06687000 & Blue Creek near Lewellen, Nebr. & 41.3369 & -102.1747 & 63 & NA & 18 & Yes & 898 & 5.20 & 3,310 & 3.88 & 5.53 \\
\hline 06707000 & North Fork South Platte River at South Platte, Colo. & 39.4089 & -105.1758 & 72 & NA & NA & No & 476 & 17.5 & 6,091 & 2.56 & 31.5 \\
\hline 06708500 & Deer Creek near Littleton, Colo. & 39.5489 & -105.1336 & 17 & NA & NA & No & 26.2 & 18.0 & 5,700 & 3.33 & 29.8 \\
\hline 06709500 & Plum Creek near Louviers, Colo. & 39.4844 & -105.0029 & 43 & NA & NA & No & 303 & 14.4 & 5,585 & 3.56 & 18.8 \\
\hline 06710385 & Bear Creek above Evergreen, Colo. & 39.6332 & -105.3369 & 30 & NA & NA & No & 103 & 16.0 & 7,080 & 2.55 & 31.8 \\
\hline 06710990 & Parmalee Gulch at Mouth, at Indian Hills, Colo. & 39.6158 & -105.2322 & 13 & NA & NA & No & 43.6 & 17.0 & 6,740 & 3.21 & 25.6 \\
\hline 06711000 & Turkey Creek near Morrison, Colo. & 39.6355 & -105.1686 & 28 & NA & NA & No & 50.2 & 18.9 & 5,718 & 3.13 & 24.9 \\
\hline 06712000 & Cherry Creek near Franktown, Colo. & 39.3558 & -104.7633 & 74 & NA & NA & Yes & 168 & 17.9 & 6,150 & 3.72 & 7.22 \\
\hline 06712500 & Cherry Creek near Melvin, Colo. & 39.6050 & -104.8225 & 30 & NA & NA & Yes & 360 & 17.4 & 5,608 & 3.64 & 8.34 \\
\hline 06718550 & North Clear Creek above Mouth near Black Hawk, Colo. & 39.7489 & -105.3997 & 19 & NA & NA & No & 60.0 & 17.0 & 6,910 & 2.46 & 30.5 \\
\hline 06719500 & Clear Creek near Golden, Colo. & 39.7505 & -105.2489 & 62 & NA & NA & Yes & 392 & 13.4 & 5,735 & 2.38 & 40.6 \\
\hline 06724000 & Saint Vrain Creek at Lyons, Colo. & 40.2180 & -105.2600 & 108 & 14 & NA & No & 216 & 12.6 & 5,292 & 3.24 & 33.7 \\
\hline 06724500 & Left Hand Creek near Boulder, Colo. & 40.1255 & -105.3039 & 16 & NA & NA & No & 52.0 & 12.6 & 5,710 & 3.23 & 36.4 \\
\hline 06726900 & Bummers Gulch near El Vado, Colo. & 40.0117 & -105.3486 & 12 & NA & NA & No & 3.90 & 14.0 & 6,270 & 3.27 & 24.3 \\
\hline 06727500 & Fourmile Creek at Orodell, Colo. & 40.0187 & -105.3262 & 22 & NA & NA & No & 24.2 & 12.6 & 5,750 & 3.12 & 36.7 \\
\hline 06730300 & Coal Creek near Plainview, Colo. & 39.8778 & -105.2772 & 23 & 20 & NA & No & 15.2 & 18.3 & 6,540 & 3.29 & 36.3 \\
\hline 06730500 & Boulder Creek at Mouth near Longmont, Colo. & 40.1522 & -105.0150 & 62 & NA & NA & No & 448 & 18.6 & 4,860 & 3.26 & 20.2 \\
\hline 06736000 & North Fork Big Thompson River at Drake, Colo. & 40.4333 & -105.3389 & 29 & 22 & NA & No & 85.1 & 12.5 & 6,170 & 3.63 & 37.4 \\
\hline 06736650 & Cedar Creek at Cedar Cove, Colo. & 40.4189 & -105.2653 & 14 & NA & NA & No & 18.9 & 16.8 & 5,600 & 4.89 & 33.0 \\
\hline 06739500 & Buckhorn Creek near Masonville, Colo. & 40.4344 & -105.1844 & 29 & NA & NA & No & 134 & 14.7 & 5,125 & 4.45 & 29.5 \\
\hline 06742000 & Little Thompson River near Berthoud, Colo. & 40.2572 & -105.2047 & 17 & NA & NA & No & 100 & 15.7 & 5,220 & 3.86 & 32.8 \\
\hline 06751490 & North Fork Cache La Poudre River at Livermore, Colo. & 40.7875 & -105.2522 & 26 & NA & NA & No & 538 & 18.3 & 5,715 & 3.03 & 19.2 \\
\hline 06755000 & South Crow Creek near Hecla, Wyo. & 41.1264 & -105.1944 & 36 & NA & NA & No & 13.5 & 16.1 & 7,130 & 3.02 & 10.2 \\
\hline 06755960 & Crow Creek at 19th Street, at Cheyenne, Wyo. & 41.1311 & -104.8286 & 23 & NA & NA & Yes & 257 & 15.7 & 6,050 & 2.93 & 9.80 \\
\hline 06758200 & Kiowa Creek at Kiowa, Colo. & 39.3372 & -104.4755 & 10 & NA & NA & Yes & 115 & 15.0 & 6,350 & 3.79 & 7.00 \\
\hline 06758700 & Middle Bijou Creek Tributary near Deer Trail, Colo. & 39.4925 & -104.1633 & 10 & NA & NA & Yes & 2.70 & 21.7 & 5,630 & 3.98 & 9.31 \\
\hline 06759100 & Bijou Creek near Fort Morgan, Colo. & 40.2828 & -103.8755 & 11 & NA & NA & No & 1,383 & 25.3 & 4,302 & 3.99 & 4.71 \\
\hline 06761900 & Lodgepole Creek Tributary near Pine Bluffs, Wyo. & 41.2564 & -104.0811 & 22 & NA & NA & No & 0.60 & 13.7 & 5,300 & 3.38 & 1.79 \\
\hline 06762500 & Lodgepole Creek at Bushnell, Nebr. & 41.2278 & -103.8931 & 72 & NA & NA & No & 1,216 & 14.1 & 4,843 & 3.15 & 4.14 \\
\hline 06762600 & Lodgepole Creek Tributary Number 2 near Albin, Wyo. & 41.3197 & -104.0808 & 25 & NA & NA & Yes & 7.60 & 15.7 & 5,330 & 3.44 & 1.99 \\
\hline 06763500 & Lodgepole Creek at Ralton, Nebr. & 41.0333 & -102.4050 & 30 & NA & 7 & Yes & 3,307 & 16.7 & 3,590 & 3.72 & 3.25 \\
\hline 06767200 & North Fork Plum Creek Tributary near Farnam, Nebr. & 40.7050 & -100.2404 & 27 & NA & NA & No & 1.83 & 22.1 & 2,737 & 4.76 & 7.39 \\
\hline 06821300 & North Fork Arikaree Tributary near Shaw, Colo. & 39.5200 & -103.4436 & 11 & NA & NA & No & 6.80 & 25.1 & 5,230 & 4.24 & 2.65 \\
\hline 06821400 & North Fork Black Wolf Creek near Vernon, Colo. & 39.9067 & -102.2694 & 11 & NA & NA & No & 19.8 & 21.4 & 3,680 & 4.37 & 2.23 \\
\hline 06821500 & Arikaree River at Haigler, Nebr. & 40.0292 & -101.9675 & 82 & NA & NA & No & 1,700 & 18.8 & 3,251 & 4.27 & 3.01 \\
\hline 06823000 & North Fork Republican River at Colorado-Nebraska State Line & 40.0694 & -102.0513 & 83 & NA & NA & No & 2,370 & 10.9 & 3,336 & 4.05 & 3.03 \\
\hline
\end{tabular}


Table 1. Summary of the streamgages used in the regression analysis of natural streams in eastern Colorado, 2015.-Continued

[USGS, U.S. Geological Survey; CDWR, Colorado Division of Water Resources; NDNR, Nebraska Department of Natural Resources; A, drainage area; mi², square miles; C, amount of soil consisting of clay in basin; \%, percent; $E_{\text {out }}$, elevation of basin outlet; ft, feet; ${ }_{6} P_{100}$, mean basin 6-hour, 100-year precipitation; in., inches; S, mean basin slope; Wyo., Wyoming; Nebr., Nebraska; Colo., Colorado; Kans., Kansas; Okla., Oklahoma; N. Mex., New Mexico; Tex., Texas; NA, not applicable]

\begin{tabular}{|c|c|c|c|c|c|c|c|c|c|c|c|c|}
\hline $\begin{array}{c}\text { USGS } \\
\text { streamgage } \\
\text { number }\end{array}$ & Streamgage name & $\begin{array}{l}\text { Latitude } \\
\text { (decimal } \\
\text { degrees) }\end{array}$ & $\begin{array}{r}\text { Longitude } \\
\text { (decimal } \\
\text { degrees) }\end{array}$ & $\begin{array}{l}\text { USGS } \\
\text { peaks }\end{array}$ & $\begin{array}{l}\text { CDWR } \\
\text { peaks }\end{array}$ & $\begin{array}{l}\text { NDNR } \\
\text { peaks }\end{array}$ & $\begin{array}{l}\text { Paleo- } \\
\text { flood } \\
\text { data }\end{array}$ & $\underset{\left(\mathrm{mi}^{2}\right)}{\mathbf{A}}$ & $\begin{array}{c}\text { C } \\
(\%)\end{array}$ & $\begin{array}{l}E_{\text {out }} \\
\text { (ft) }\end{array}$ & $\begin{array}{l}{ }_{6} P_{100} \\
\text { (in.) }\end{array}$ & $\begin{array}{c}\mathbf{S} \\
(\%)\end{array}$ \\
\hline 06823500 & Buffalo Creek near Haigler, Nebr. & 40.0394 & -101.8667 & 73 & NA & NA & No & 177 & 6.59 & 3,189 & 4.26 & 4.32 \\
\hline 06825000 & South Fork Republican River near Idalia, Colo. & 39.6164 & -102.2427 & 32 & NA & NA & No & 1,412 & 21.7 & 3,680 & 4.56 & 2.77 \\
\hline 06825500 & Landsman Creek near Hale, Colo. & 39.5755 & -102.2521 & 26 & NA & NA & No & 269 & 23.7 & 3,720 & 4.63 & 2.98 \\
\hline 06826900 & Sand Creek near Hale, Colo. & 39.6972 & -102.1774 & 11 & NA & NA & No & 14.6 & 21.3 & 3,750 & 4.56 & 2.09 \\
\hline 06829700 & Thompson Canyon near Trenton, Nebr. & 40.1622 & -100.9590 & 13 & NA & NA & No & 9.10 & 22.0 & 2,670 & 4.52 & 10.6 \\
\hline 06831500 & Frenchman Creek near Imperial, Nebr. & 40.4317 & -101.6269 & 31 & NA & NA & Yes & 1,050 & 17.8 & 3,130 & 4.28 & 2.06 \\
\hline 06835000 & Stinking Water Creek near Palisade, Nebr. & 40.3731 & -101.1169 & 23 & NA & NA & No & 1,500 & 15.0 & 2,741 & 4.46 & 3.54 \\
\hline 06835100 & Bobtail Creek near Palisade, Nebr. & 40.3147 & -101.1116 & 13 & NA & NA & Yes & 30.2 & 22.0 & 2,920 & 4.38 & 8.38 \\
\hline 06836000 & Blackwood Creek near Culbertson, Nebr. & 40.2361 & -100.8111 & 42 & NA & NA & No & 320 & 20.7 & 2,555 & 4.44 & 8.91 \\
\hline 06836500 & Driftwood Creek near McCook, Nebr. & 40.1458 & -100.6732 & 33 & NA & NA & No & 361 & 21.7 & 2,503 & 4.61 & 6.30 \\
\hline 06838200 & Coon Creek at Indianola, Nebr. & 40.2342 & -100.4274 & 37 & NA & NA & No & 69.0 & 21.8 & 2,370 & 4.65 & 6.74 \\
\hline 06838550 & Dry Creek at Bartley, Nebr. & 40.2506 & -100.3176 & 23 & NA & NA & No & 42.0 & 21.8 & 2,320 & 4.75 & 6.26 \\
\hline 06839200 & Elkhorn Canyon near Maywood, Nebr. & 40.6028 & -100.7010 & 27 & NA & NA & No & 6.70 & 21.6 & 2,780 & 4.52 & 19.0 \\
\hline 06839600 & Frazier Creek near Maywood, Nebr. & 40.5847 & -100.6296 & 19 & NA & NA & No & 11.3 & 21.9 & 2,722 & 4.56 & 13.6 \\
\hline 06839850 & Fox Creek north of Curtis, Nebr. & 40.8264 & -100.5240 & 19 & NA & NA & No & 13.8 & 21.6 & 2,760 & 4.54 & 18.6 \\
\hline 06839900 & Fox Creek above Cut Canyon near Curtis, Nebr. & 40.7445 & -100.5315 & 28 & NA & NA & No & 31.8 & 21.6 & 2,640 & 4.55 & 18.8 \\
\hline 06839950 & Cut Canyon near Curtis, Nebr. & 40.7275 & -100.5365 & 28 & NA & NA & No & 25.6 & 21.7 & 2,625 & 4.53 & 21.9 \\
\hline 06840000 & Fox Creek at Curtis, Nebr. & 40.6347 & -100.4903 & 34 & NA & NA & No & 74.5 & 21.7 & 2,520 & 4.55 & 18.7 \\
\hline 06840500 & Dry Creek near Curtis, Nebr. & 40.6425 & -100.4450 & 20 & NA & NA & No & 21.6 & 22.1 & 2,548 & 4.63 & 13.7 \\
\hline 06841000 & Medicine Creek above Harry Strunk Lake, Nebr. & 40.5006 & -100.3228 & 21 & NA & NA & No & 770 & 16.9 & 2,381 & 4.54 & 11.6 \\
\hline 06841500 & Mitchell Creek above Harry Strunk Lake, Nebr. & 40.4719 & -100.2575 & 25 & NA & NA & No & 52.0 & 22.6 & 2,377 & 4.72 & 8.06 \\
\hline 06844700 & South Fork Sappa Creek near Brewster, Kans. & 39.2853 & -101.4660 & 22 & NA & NA & No & 85.7 & 20.1 & 3,440 & 4.77 & 1.57 \\
\hline 06844800 & South Fork Sappa Creek Tributary near Goodland, Kans. & 39.3206 & -101.6329 & 33 & NA & NA & Yes & 20.1 & 19.7 & 3,590 & 4.74 & 1.00 \\
\hline 06844900 & South Fork Sappa Creek near Achilles, Kans. & 39.6769 & -100.7221 & 50 & NA & NA & No & 446 & 20.5 & 2,722 & 4.85 & 2.61 \\
\hline 06845000 & Sappa Creek near Oberlin, Kans. & 39.8131 & -100.5343 & 45 & NA & NA & No & 1,063 & 20.8 & 2,538 & 4.83 & 3.16 \\
\hline 06845100 & Long Branch draw near Norcatur, Kans. & 39.9017 & -100.1790 & 53 & NA & NA & No & 31.7 & 21.9 & 2,440 & 4.84 & 4.73 \\
\hline 06846200 & Beaver Creek Tributary near Ludell, Kans. & 39.8147 & -100.8724 & 33 & NA & NA & No & 10.2 & 21.3 & 2,840 & 4.71 & 5.08 \\
\hline 06846500 & Beaver Creek at Cedar Bluffs, Kans. & 39.9850 & -100.5601 & 68 & NA & NA & No & 1,620 & 21.7 & 2,520 & 4.73 & 3.77 \\
\hline 06847600 & Prairie Dog Creek Tributary at Colby, Kans. & 39.3911 & -101.0457 & 56 & NA & NA & No & 7.53 & 19.7 & 3,130 & 4.92 & 1.59 \\
\hline 06847900 & Prairie Dog Creek above Keith Sebelius Lake, Kans. & 39.7697 & -100.1007 & 52 & NA & NA & No & 590 & 21.2 & 2,335 & 4.88 & 3.70 \\
\hline 06858500 & North Fork Smoky Hill River near McAllaster, Kans. & 39.0179 & -101.3479 & 33 & NA & NA & Yes & 696 & 22.5 & 3,070 & 4.63 & 2.58 \\
\hline 06859500 & Ladder Creek below Chalk Creek near Scott City, Kans. & 38.7889 & -100.8699 & 32 & NA & NA & No & 1,460 & 23.4 & 2,638 & 4.57 & 2.13 \\
\hline 06860000 & Smoky Hill River at Elkader, Kans. & 38.7947 & -100.8585 & 75 & NA & NA & No & 3,560 & 23.0 & 2,623 & 4.61 & 2.69 \\
\hline 06860300 & South Branch Hackberry Creek near Orion, Kans. & 38.9417 & -100.7032 & 12 & NA & NA & No & 49.6 & 20.2 & 2,840 & 4.85 & 1.55 \\
\hline 06860500 & Hackberry Creek near Gove, Kans. & 38.9536 & -100.4882 & 39 & NA & NA & No & 426 & 21.3 & 2,593 & 4.91 & 2.43 \\
\hline 07093775 & Badger Creek, Lower Station, near Howard, Colo. & 38.4672 & -105.8600 & 23 & NA & NA & No & 211 & 21.0 & 6,780 & 2.71 & 22.5 \\
\hline 07095000 & Grape Creek near Westcliffe, Colo. & 38.1861 & -105.4836 & 69 & 17 & NA & No & 321 & 16.2 & 7,690 & 3.42 & 19.3 \\
\hline 07096500 & Fourmile Creek near Canon City, Colo. & 38.4364 & -105.1914 & 32 & NA & NA & No & 436 & 19.1 & 5,254 & 3.19 & 26.0 \\
\hline 07099060 & Beaver Creek above Highway 115 near Penrose, Colo. & 38.4892 & -104.9975 & 23 & NA & NA & No & 138 & 17.3 & 5,660 & 3.37 & 34.9 \\
\hline
\end{tabular}


Table 1. Summary of the streamgages used in the regression analysis of natural streams in eastern Colorado, 2015.-Continued

[USGS, U.S. Geological Survey; CDWR, Colorado Division of Water Resources; NDNR, Nebraska Department of Natural Resources; A, drainage area; mi² , square miles; C, amount of soil consisting of clay in basin; \%, percent; $E_{\text {out }}$, elevation of basin outlet; ft, feet; ${ }_{6} P_{100}$, mean basin 6-hour, 100-year precipitation; in., inches; S, mean basin slope; Wyo., Wyoming; Nebr., Nebraska; Colo., Colorado; Kans., Kansas; Okla., Oklahoma; N. Mex., New Mexico; Tex., Texas; NA, not applicable]

\begin{tabular}{|c|c|c|c|c|c|c|c|c|c|c|c|c|}
\hline $\begin{array}{c}\text { USGS } \\
\text { streamgage } \\
\text { number }\end{array}$ & Streamgage name & $\begin{array}{l}\text { Latitude } \\
\text { (decimal } \\
\text { degrees) }\end{array}$ & $\begin{array}{l}\text { Longitude } \\
\text { (decimal } \\
\text { degrees) }\end{array}$ & $\begin{array}{l}\text { USGS } \\
\text { peaks }\end{array}$ & $\begin{array}{l}\text { CDWR } \\
\text { peaks }\end{array}$ & $\begin{array}{l}\text { NDNR } \\
\text { peaks }\end{array}$ & $\begin{array}{l}\text { Paleo- } \\
\text { flood } \\
\text { data }\end{array}$ & $\underset{\left(\mathrm{mi}^{2}\right)}{\mathrm{A}}$ & $\begin{array}{l}\text { C } \\
(\%)\end{array}$ & $\begin{array}{l}E_{\text {out }} \\
\text { (ft) }\end{array}$ & $\begin{array}{l}{ }_{6} P_{100} \\
\text { (in.) }\end{array}$ & $\begin{array}{c}\mathbf{S} \\
(\%)\end{array}$ \\
\hline 07099080 & Red Creek below Sullivan Park at Fort Carson, Colo. & 38.4997 & -104.9133 & 13 & NA & NA & No & 26.6 & 21.4 & 5,783 & 3.94 & 18.5 \\
\hline 07099215 & Turkey Creek near Fountain, Colo. & 38.6117 & -104.8947 & 30 & NA & NA & Yes & 13.0 & 22.5 & 6,420 & 3.97 & 44.5 \\
\hline 07099220 & Little Turkey Creek near Fountain, Colo. & 38.6272 & -104.8664 & 10 & NA & NA & Yes & 9.59 & 22.4 & 6,395 & 4.02 & 39.5 \\
\hline 07099230 & Turkey Creek above Teller Reservoir near Stone City, Colo. & 38.4650 & -104.8272 & 35 & NA & NA & No & 62.2 & 21.8 & 5,520 & 3.98 & 30.9 \\
\hline 07103700 & Fountain Creek near Colorado Springs, Colo. & 38.8547 & -104.8780 & 56 & NA & NA & No & 102 & 11.6 & 6,110 & 3.34 & 37.5 \\
\hline 07103703 & Camp Creek at Garden of the Gods, Colo. & 38.8769 & -104.8728 & 22 & NA & NA & Yes & 9.38 & 14.1 & 6,310 & 3.84 & 43.1 \\
\hline 07103747 & Monument Creek at Palmer Lake, Colo. & 39.1026 & -104.8923 & 20 & NA & NA & No & 25.7 & 9.87 & 6,950 & 3.62 & 32.1 \\
\hline 07103960 & Kettle Creek above U.S. Air Force Academy, Colo. & 38.9761 & -104.7986 & 13 & NA & NA & Yes & 16.2 & 16.1 & 6,620 & 3.92 & 7.92 \\
\hline 07103977 & Cottonwood Creek at Cowpoke Road at Colorado Springs, Colo. & 38.9511 & -104.7136 & 11 & NA & NA & Yes & 5.91 & 14.9 & 6,875 & 3.97 & 6.09 \\
\hline 07104000 & Monument Creek at Pikeview, Colo. & 38.9178 & -104.8186 & 49 & NA & NA & No & 203 & 13.7 & 6,203 & 3.82 & 16.2 \\
\hline 07104050 & $\begin{array}{l}\text { North Rockrimmon Creek above Delmonico Drive at Colorado } \\
\text { Springs, Colo. }\end{array}$ & 38.9155 & -104.8258 & 12 & NA & NA & No & 1.83 & 11.0 & 6,240 & 3.97 & 13.3 \\
\hline 07105000 & Bear Creek near Colorado Springs, Colo. & 38.8225 & -104.8886 & 22 & NA & NA & Yes & 6.89 & 12.6 & 6,520 & 3.66 & 48.6 \\
\hline 07105490 & Cheyenne Creek at Evans Avenue at Colorado Springs, Colo. & 38.7905 & -104.8641 & 22 & NA & NA & Yes & 21.7 & 11.1 & 6,280 & 3.49 & 48.7 \\
\hline 07105900 & Jimmy Camp Creek at Fountain, Colo. & 38.6844 & -104.6886 & 39 & NA & NA & No & 65.4 & 34.5 & 5,530 & 4.22 & 6.40 \\
\hline 07105920 & $\begin{array}{l}\text { Little Fountain Creek above Keaton Reservoir, near Fort Carson, } \\
\text { Colo. }\end{array}$ & 38.6817 & -104.8586 & 13 & NA & NA & No & 11.0 & 21.9 & 6,430 & 3.78 & 48.2 \\
\hline 07105940 & Little Fountain Creek near Fountain, Colo. & 38.6431 & -104.7467 & 12 & NA & NA & Yes & 26.9 & 27.9 & 5,566 & 4.00 & 24.0 \\
\hline 07105945 & Rock Creek above Fort Carson Reservation, Colo. & 38.7075 & -104.8466 & 35 & NA & NA & No & 6.80 & 22.8 & 6,390 & 3.91 & 48.2 \\
\hline 07106500 & Fountain Creek at Pueblo, Colo. & 38.2878 & -104.6011 & 74 & NA & NA & No & 925 & 22.4 & 4,705 & 3.89 & 7.49 \\
\hline 07107500 & St. Charles River at Burnt Mill, Colo. & 38.0517 & -104.7936 & 27 & NA & NA & Yes & 171 & 22.8 & 5,350 & 4.12 & 28.4 \\
\hline 07108900 & St. Charles River at Vineland, Colo. & 38.2456 & -104.4864 & 36 & NA & NA & No & 474 & 24.9 & 4,582 & 3.78 & 11.6 \\
\hline 07111000 & Huerfano River at Manzanares Crossing, near Redwing, Colo. & 37.7278 & -105.3514 & 58 & 18 & NA & No & 73.0 & 17.2 & 8,270 & 3.15 & 31.8 \\
\hline 07112500 & Huerfano River at Badito, Colo. & 37.7272 & -105.0125 & 22 & 6 & NA & No & 532 & 19.6 & 6,415 & 3.28 & 20.8 \\
\hline 07114000 & Cucharas River at Boyd Ranch near La Veta, Colo. & 37.4200 & -105.0528 & 47 & 18 & NA & No & 53.1 & 20.1 & 7,781 & 3.37 & 35.3 \\
\hline 07116000 & Huerfano River below Huerfano Valley Dam near Undercliffe, Colo. & 38.0014 & -104.4725 & 28 & NA & NA & No & 1,673 & 23.9 & 4,886 & 3.34 & 11.7 \\
\hline 07118000 & Apishapa River near Aguilar, Colo. & 37.3864 & -104.6658 & 12 & NA & NA & No & 140 & 24.3 & 6,408 & 3.42 & 26.8 \\
\hline 07120620 & Big Arroyo near Thatcher, Colo. & 37.5548 & -104.0212 & 30 & NA & NA & Yes & 15.6 & 26.0 & 5,288 & 3.82 & 6.14 \\
\hline 07121500 & Timpas Creek at Mouth near Swink, Colo. & 38.0031 & -103.6561 & 51 & NA & NA & No & 511 & 24.9 & 4,120 & 3.79 & 3.86 \\
\hline 07122400 & Crooked Arroyo near Swink, Colo. & 37.9822 & -103.5983 & 26 & 18 & NA & No & 108 & 25.9 & 4,100 & 3.94 & 2.14 \\
\hline 07123675 & Horse Creek near Las Animas, Colo. & 38.0850 & -103.3538 & 29 & NA & NA & No & 1,403 & 21.6 & 3,975 & 3.99 & 2.01 \\
\hline 07124300 & Long Canyon Creek near Madrid, Colo. & 37.1147 & -104.6053 & 18 & NA & NA & Yes & 100 & 36.8 & 6,259 & 3.53 & 25.4 \\
\hline 07124500 & Purgatoire River at Trinidad, Colo. & 37.1709 & -104.5092 & 70 & NA & NA & No & 749 & 30.5 & 5,980 & 3.35 & 28.0 \\
\hline 07125100 & Frijole Creek near Alfalfa, Colo. & 37.1997 & -104.2110 & 15 & NA & NA & Yes & 79.1 & 27.3 & 5,420 & 3.63 & 10.0 \\
\hline 07125500 & San Francisco Creek near Alfalfa, Colo. & 37.1849 & -104.1453 & 15 & NA & NA & Yes & 162 & 28.9 & 5,320 & 3.78 & 13.4 \\
\hline 07126100 & Luning Arroyo near Model, Colo. & 37.3045 & -104.0155 & 16 & NA & NA & No & 84.8 & 25.6 & 5,150 & 3.71 & 2.83 \\
\hline 07126140 & Van Bremer Arroyo near Tyrone, Colo. & 37.3995 & -104.1158 & 31 & NA & NA & No & 119 & 22.9 & 5,310 & 3.69 & 2.07 \\
\hline 07126200 & Van Bremer Arroyo near Model, Colo. & 37.3456 & -103.9580 & 48 & NA & NA & Yes & 161 & 23.3 & 4,960 & 3.72 & 4.81 \\
\hline 07126325 & Taylor Arroyo below Rock Crossing, near Thatcher, Colo. & 37.4242 & -103.9202 & 31 & NA & NA & Yes & 48.7 & 25.5 & 4,982 & 3.83 & 4.43 \\
\hline 07126390 & Lockwood Canyon Creek near Thatcher, Colo. & 37.4928 & -103.8280 & 30 & NA & NA & Yes & 48.6 & 23.6 & 4,785 & 3.89 & 4.59 \\
\hline
\end{tabular}


Table 1. Summary of the streamgages used in the regression analysis of natural streams in eastern Colorado, 2015.-Continued

[USGS, U.S. Geological Survey; CDWR, Colorado Division of Water Resources; NDNR, Nebraska Department of Natural Resources; A, drainage area; mi², square miles; C, amount of soil consisting of clay in basin; \%, percent; $E_{\text {out }}$, elevation of basin outlet; ft, feet; ${ }_{6}{ }_{100}$, mean basin 6-hour, 100-year precipitation; in., inches; S, mean basin slope; Wyo., Wyoming; Nebr., Nebraska; Colo., Colorado; Kans., Kansas; Okla., Oklahoma; N. Mex., New Mexico; Tex., Texas; NA, not applicable]

\begin{tabular}{|c|c|c|c|c|c|c|c|c|c|c|c|c|}
\hline $\begin{array}{c}\text { USGS } \\
\text { streamgage } \\
\text { number }\end{array}$ & Streamgage name & $\begin{array}{l}\text { Latitude } \\
\text { (decimal } \\
\text { degrees) }\end{array}$ & $\begin{array}{r}\text { Longitude } \\
\text { (decimal } \\
\text { degrees) }\end{array}$ & $\begin{array}{l}\text { USGS } \\
\text { peaks }\end{array}$ & $\begin{array}{l}\text { CDWR } \\
\text { peaks }\end{array}$ & $\begin{array}{l}\text { NDNR } \\
\text { peaks }\end{array}$ & $\begin{array}{l}\text { Paleo- } \\
\text { flood } \\
\text { data }\end{array}$ & $\underset{\left(\mathrm{mi}^{2}\right)}{\mathrm{A}}$ & $\begin{array}{c}\text { C } \\
(\%)\end{array}$ & $\begin{array}{l}E_{\text {out }} \\
\text { (ft) }\end{array}$ & $\begin{array}{l}{ }_{6} \boldsymbol{P}_{100} \\
\text { (in.) }\end{array}$ & $\begin{array}{c}\text { S } \\
(\%)\end{array}$ \\
\hline 07126415 & Red Rock Canyon Creek at Mouth near Thatcher, Colo. & 37.5153 & -103.7255 & 31 & NA & NA & Yes & 48.7 & 18.1 & 4,510 & 3.98 & 11.1 \\
\hline 07126470 & Chacuaco Creek at Mouth near Timpas, Colo. & 37.5439 & -103.6322 & 16 & NA & NA & No & 421 & 19.9 & 4,410 & 4.30 & 8.55 \\
\hline 07126480 & Bent Canyon Creek at Mouth near Timpas, Colo. & 37.5892 & -103.6483 & 29 & NA & NA & Yes & 56.1 & 20.5 & 4,402 & 3.98 & 11.2 \\
\hline 07126500 & Purgatoire River at Ninemile Dam, near Higbee, Colo. & 37.7147 & -103.5111 & 71 & 17 & NA & No & 2,875 & 25.2 & 4,241 & 3.77 & 8.93 \\
\hline 07133200 & Clay Creek Tributary near Deora, Colo. & 37.7242 & -102.7405 & 11 & NA & NA & No & 2.40 & 22.5 & 4,285 & 4.82 & 5.05 \\
\hline 07134100 & Big Sandy Creek near Lamar, Colo. & 38.1142 & -102.4838 & 34 & NA & NA & Yes & 3,216 & 21.4 & 3,545 & 4.33 & 2.57 \\
\hline 07134990 & Wild Horse Creek above Holly, Colo. & 38.0570 & -102.1385 & 18 & NA & NA & No & 175 & 23.9 & 3,405 & 4.58 & 1.62 \\
\hline 07135000 & Two Butte Creek near Holly, Colo. & 38.0278 & -102.1391 & 10 & NA & NA & Yes & 817 & 21.6 & 3,415 & 4.87 & 3.57 \\
\hline 07138600 & White Woman Creek Tributary near Selkirk, Kans. & 38.5250 & -101.6216 & 39 & NA & NA & Yes & 25.2 & 26.0 & 3,510 & 4.45 & 1.16 \\
\hline 07138650 & White Woman Creek near Leoti, Kans. & 38.4811 & -101.4882 & 20 & NA & NA & Yes & 500 & 24.1 & 3,320 & 4.50 & 1.36 \\
\hline 07138800 & Lion Creek Tributary near Modoc, Kans. & 38.4800 & -101.0629 & 21 & NA & NA & No & 7.00 & 26.5 & 3,080 & 4.55 & 0.48 \\
\hline 07140600 & Pawnee River Tributary near Kalvesta, Kans. & 38.0617 & -100.3504 & 33 & NA & NA & No & 6.89 & 33.7 & 2,650 & 5.01 & 1.14 \\
\hline 07141400 & South Fork Walnut Creek Tributary near Dighton, Kans. & 38.4828 & -100.4154 & 21 & NA & NA & No & 0.81 & 28.6 & 2,700 & 5.10 & 1.57 \\
\hline 07153500 & Dry Cimarron River near Guy, N. Mex. & 36.9875 & -103.4241 & 33 & NA & NA & No & 500 & 21.7 & 4,900 & 4.10 & 13.5 \\
\hline 07154400 & Carrizozo Creek near Kenton, Okla. & 36.8833 & -103.0183 & 61 & NA & NA & No & 112 & 20.2 & 4,380 & 4.44 & 11.8 \\
\hline 07154500 & Cimarron River near Kenton, Okla. & 36.9267 & -102.9591 & 63 & NA & NA & No & 1,208 & 21.0 & 4,262 & 4.29 & 13.2 \\
\hline 07154650 & Tesesquite Creek near Kenton, Okla. & 36.8978 & -102.9016 & 22 & NA & NA & No & 24.9 & 16.7 & 4,250 & 5.07 & 12.3 \\
\hline 07155100 & Cold Springs Creek near Wheeless, Okla. & 36.7722 & -102.8049 & 18 & NA & NA & Yes & 10.6 & 28.7 & 4,450 & 4.99 & 1.86 \\
\hline 07155590 & Cimarron River near Elkhart, Kans. & 37.1220 & -101.8979 & 41 & NA & NA & No & 2,927 & 19.6 & 3,377 & 4.65 & 5.38 \\
\hline 07155900 & North Fork Cimarron River Tributary near Elkhart, Kans. & 37.1909 & -101.8988 & 33 & NA & NA & No & 57.1 & 26.7 & 3,490 & 4.59 & 0.58 \\
\hline 07156000 & North Fork Cimarron River Tributary near Richfield, Kans. & 37.3113 & -101.7736 & 21 & NA & NA & No & 103 & 24.9 & 3,350 & 4.58 & 0.81 \\
\hline 07156010 & North Fork Cimarron River at Richfield, Kans. & 37.2584 & -101.7754 & 15 & NA & NA & No & 411 & 21.4 & 3,344 & 4.78 & 0.91 \\
\hline 07156100 & Sand Arroyo Creek near Johnson, Kans. & 37.5015 & -101.7616 & 15 & NA & NA & Yes & 584 & 19.7 & 3,328 & 5.02 & 1.57 \\
\hline 07156220 & Bear Creek near Johnson, Kans. & 37.6285 & -101.7616 & 32 & NA & NA & Yes & 813 & 27.0 & 3,292 & 4.88 & 1.51 \\
\hline 07156600 & Cimarron River Tributary near Moscow, Kans. & 37.3363 & -101.0504 & 33 & NA & NA & Yes & 21.5 & 23.8 & 2,830 & 4.68 & 1.71 \\
\hline 07156700 & Cimarron River Tributary near Satanta, Kans. & 37.2709 & -100.9271 & 49 & NA & NA & No & 2.41 & 22.0 & 2,740 & 4.97 & 1.92 \\
\hline 07157100 & Crooked Creek near Copeland, Kans. & 37.5653 & -100.5546 & 33 & NA & NA & No & 44.0 & 29.6 & 2,695 & 4.97 & 1.14 \\
\hline 07157400 & Crooked Creek Tributary at Meade, Kans. & 37.2964 & -100.3399 & 33 & NA & NA & No & 6.57 & 30.3 & 2,450 & 5.48 & 4.12 \\
\hline 07157500 & Crooked Creek near Englewood, Kans. & 37.0325 & -100.2113 & 36 & NA & NA & No & 1,157 & 28.4 & 2,164 & 5.36 & 2.08 \\
\hline 07157550 & West Fork Creek near Knowles, Okla. & 36.8750 & -100.1226 & 22 & NA & NA & No & 4.44 & 20.2 & 2,280 & 5.60 & 6.27 \\
\hline 07199000 & Canadian River near Hebron, N. Mex. & 36.7872 & -104.4622 & 40 & NA & NA & No & 199 & 37.5 & 6,248 & 3.62 & 23.6 \\
\hline 07201000 & Raton Creek at Raton, N. Mex. & 36.9058 & -104.4361 & 54 & NA & NA & No & 14.6 & 32.6 & 6,640 & 3.94 & 23.4 \\
\hline 07201200 & Chicorica Creek Tributary near Raton, N. Mex. & 36.8308 & -104.3333 & 31 & NA & NA & Yes & 5.20 & 31.4 & 6,480 & 3.88 & 16.3 \\
\hline 07201450 & Green Mountain Arroyo near Raton, N. Mex. & 36.7842 & -104.2640 & 12 & NA & NA & Yes & 18.2 & 30.6 & 6,499 & 3.84 & 10.4 \\
\hline 07202000 & Chicorica Creek near Hebron, N. Mex. & 36.7703 & -104.3964 & 10 & NA & NA & No & 381 & 33.3 & 6,200 & 3.88 & 13.5 \\
\hline 07203000 & Vermejo River near Dawson, N. Mex. & 36.6810 & -104.7864 & 85 & NA & NA & No & 295 & 35.0 & 6,360 & 3.33 & 24.7 \\
\hline 07203600 & Rio Del Plano Tributary near Taylor Springs, N. Mex. & 36.4498 & -104.3767 & 12 & NA & NA & Yes & 6.71 & 32.6 & 6,148 & 3.64 & 2.03 \\
\hline 07204000 & Moreno Creek at Eagle Nest, N. Mex. & 36.5539 & -105.2680 & 72 & NA & NA & No & 73.8 & 23.9 & 8,197 & 3.26 & 26.3 \\
\hline 07204500 & Cieneguilla Creek near Eagle Nest, N. Mex. & 36.4852 & -105.2654 & 72 & NA & NA & No & 56.0 & 29.1 & 8,200 & 2.98 & 19.5 \\
\hline 07205000 & Sixmile Creek near Eagle Nest, N. Mex. & 36.5185 & -105.2752 & 76 & NA & NA & No & 10.5 & 24.1 & 8,195 & 3.13 & 26.9 \\
\hline
\end{tabular}


Table 1. Summary of the streamgages used in the regression analysis of natural streams in eastern Colorado, 2015.-Continued

[USGS, U.S. Geological Survey; CDWR, Colorado Division of Water Resources; NDNR, Nebraska Department of Natural Resources; A, drainage area; mi² , square miles; C, amount of soil consisting of clay in basin; \%, percent; $E_{\text {out }}$, elevation of basin outlet; ft, feet; ${ }_{6} P_{100}$, mean basin 6-hour, 100-year precipitation; in., inches; S, mean basin slope; Wyo., Wyoming; Nebr., Nebraska; Colo., Colorado; Kans., Kansas Okla., Oklahoma; N. Mex., New Mexico; Tex., Texas; NA, not applicable]

\begin{tabular}{|c|c|c|c|c|c|c|c|c|c|c|c|c|}
\hline $\begin{array}{c}\text { USGS } \\
\text { streamgage } \\
\text { number }\end{array}$ & Streamgage name & $\begin{array}{l}\text { Latitude } \\
\text { (decimal } \\
\text { degrees) }\end{array}$ & $\begin{array}{r}\text { Longitude } \\
\text { (decimal } \\
\text { degrees) }\end{array}$ & $\begin{array}{l}\text { USGS } \\
\text { peaks }\end{array}$ & $\begin{array}{l}\text { CDWR } \\
\text { peaks }\end{array}$ & $\begin{array}{l}\text { NDNR } \\
\text { peaks }\end{array}$ & $\begin{array}{l}\text { Paleo- } \\
\text { flood } \\
\text { data }\end{array}$ & $\underset{\left(\mathrm{mi}^{2}\right)}{\mathrm{A}}$ & $\begin{array}{c}\text { C } \\
(\%)\end{array}$ & $\begin{array}{l}E_{\text {out }} \\
\text { (ft) }\end{array}$ & $\begin{array}{l}{ }_{6} \boldsymbol{P}_{100} \\
\text { (in.) }\end{array}$ & $\begin{array}{c}\mathbf{S} \\
(\%)\end{array}$ \\
\hline 07206400 & Clear Creek near Ute Park, N. Mex. & 36.5264 & -105.1750 & 48 & NA & NA & No & 7.44 & 20.7 & 7,860 & 3.27 & 48.1 \\
\hline 07207500 & Ponil Creek near Cimarron, N. Mex. & 36.5737 & -104.9468 & 62 & NA & NA & No & 171 & 33.4 & 6,630 & 3.30 & 27.3 \\
\hline 07208500 & Rayado Creek near Cimarron, N. Mex. & 36.3723 & -104.9693 & 91 & NA & NA & No & 60.1 & 29.1 & 6,720 & 3.46 & 24.1 \\
\hline 07211500 & Canadian River near Taylor Springs, N. Mex. & 36.2976 & -104.4955 & 73 & NA & NA & No & 2,850 & 33.5 & 5,640 & 3.49 & 15.4 \\
\hline 07213700 & Canadian River Tributary near Mills, N. Mex. & 36.1667 & -104.2636 & 13 & NA & NA & No & 4.20 & 31.7 & 5,908 & 3.95 & 5.34 \\
\hline 07214800 & Rio La Casa near Cleveland, N. Mex. & 35.9742 & -105.3892 & 14 & NA & NA & No & 23.0 & 25.3 & 7,635 & 4.13 & 33.5 \\
\hline 07220000 & Sapello River at Sapello, N. Mex. & 35.7698 & -105.2520 & 17 & NA & NA & No & 132 & 32.4 & 6,910 & 3.94 & 24.1 \\
\hline 07220900 & Dog Creek near Shoemaker, N. Mex. & 35.8261 & -104.8881 & 57 & NA & NA & No & 18.4 & 30.1 & 6,300 & 3.64 & 6.00 \\
\hline 07221600 & Lagartija Creek Tributary near Sanchez, N. Mex. & 35.6386 & -104.4156 & 24 & NA & NA & No & 1.16 & 23.7 & 4,605 & 3.85 & 13.6 \\
\hline 07222300 & Trementina Creek at Trementina, N. Mex. & 35.4672 & -104.4167 & 52 & NA & NA & No & 63.9 & 23.2 & 4,580 & 3.96 & 17.0 \\
\hline 07222500 & Conchas River at Variadero, N. Mex. & 35.4028 & -104.4436 & 60 & NA & NA & No & 523 & 27.0 & 4,430 & 3.75 & 7.64 \\
\hline 07222800 & Garita Creek Tributary near Variadero, N. Mex. & 35.3358 & -104.3639 & 39 & NA & NA & No & 12.0 & 23.1 & 4,290 & 4.02 & 4.04 \\
\hline 07225300 & Bluewater Creek near Tucumcari, N. Mex. & 35.1419 & -103.7928 & 40 & NA & NA & No & 15.2 & 21.6 & 4,100 & 4.41 & 6.12 \\
\hline 07225500 & Ute Creek near Gladstone, N. Mex. & 36.3059 & -103.9255 & 12 & NA & NA & No & 269 & 26.5 & 5,800 & 4.07 & 6.16 \\
\hline 07226200 & Bueyeros Creek at Bueyeros, N. Mex. & 35.9797 & -103.6856 & 34 & NA & NA & No & 34.0 & 26.7 & 4,540 & 4.27 & 11.1 \\
\hline 07226500 & Ute Creek near Logan, N. Mex. & 35.4385 & -103.5258 & 72 & NA & NA & No & 2,060 & 24.1 & 3,820 & 4.17 & 5.34 \\
\hline 07227100 & Revuelto Creek near Logan, N. Mex. & 35.3444 & -103.3896 & 54 & NA & NA & No & 786 & 25.7 & 3,660 & 4.52 & 5.52 \\
\hline 07227150 & Arroyo Del Puerto near Endee, N. Mex. & 35.0590 & -103.1022 & 23 & NA & NA & No & 25.0 & 30.4 & 3,920 & 4.82 & 5.92 \\
\hline 07227200 & Tramperos Creek near Stead, N. Mex. & 36.0708 & -103.2033 & 47 & NA & NA & No & 556 & 25.9 & 4,481 & 4.41 & 5.93 \\
\hline 07227295 & Sandy Arroyo Tributary near Clayton, N. Mex. & 36.3889 & -103.3186 & 43 & NA & NA & No & 1.20 & 25.6 & 5,110 & 4.55 & 2.01 \\
\hline 07227300 & Sand Draw near Clayton, N. Mex. & 36.3422 & -103.1842 & 18 & NA & NA & Yes & 42.0 & 27.8 & 4,800 & 4.53 & 2.36 \\
\hline 07227920 & Dixon Creek near Borger, Tex. & 35.6648 & -101.3510 & 15 & NA & NA & No & 134 & 26.2 & 2,836 & 4.90 & 5.67 \\
\hline 07232500 & Beaver River near Guymon, Okla. & 36.7214 & -101.4896 & 57 & NA & NA & No & 2,033 & 26.8 & 2,971 & 4.57 & 2.88 \\
\hline 07232550 & South Fork Tributary near Guymon, Okla. & 36.6684 & -101.4988 & 22 & NA & NA & No & 0.26 & 38.5 & 3,120 & 4.70 & 0.67 \\
\hline 07232650 & Aqua Frio Creek near Felt, Okla. & 36.5540 & -102.7866 & 12 & NA & NA & Yes & 33.9 & 29.7 & 4,410 & 4.86 & 1.09 \\
\hline 07232900 & Coldwater Creek near Guymon, Okla. & 36.5720 & -101.3815 & 30 & NA & NA & No & 1,903 & 29.9 & 2,871 & 4.78 & 1.52 \\
\hline 07233500 & Palo Duro Creek near Spearman, Tex. & 36.2023 & -101.3060 & 49 & NA & NA & No & 625 & 35.4 & 2,962 & 4.75 & 1.11 \\
\hline 07233850 & Sharp Creek Tributary near Turpin, Okla. & 36.8639 & -100.9129 & 12 & NA & NA & No & 1.00 & 24.0 & 2,760 & 5.36 & 0.40 \\
\hline 07234050 & North Fork Clear Creek Tributary near Balko, Okla. & 36.6170 & -100.6643 & 22 & NA & NA & No & 4.30 & 30.9 & 2,770 & 5.19 & 1.96 \\
\hline 07234100 & Clear Creek near Elmwood, Okla. & 36.6450 & -100.5024 & 24 & NA & NA & No & 170 & 32.2 & 2,541 & 5.17 & 2.55 \\
\hline 07301410 & Sweetwater Creek near Kelton, Tex. & 35.4731 & -100.1210 & 52 & NA & NA & No & 297 & 18.6 & 2,230 & 5.33 & 3.42 \\
\hline NA & Raton Creek above Starkville, Colo. & 37.1306 & -104.5214 & 0 & 10 & NA & No & 54.7 & 35.9 & 6,240 & 3.77 & 28.0 \\
\hline
\end{tabular}



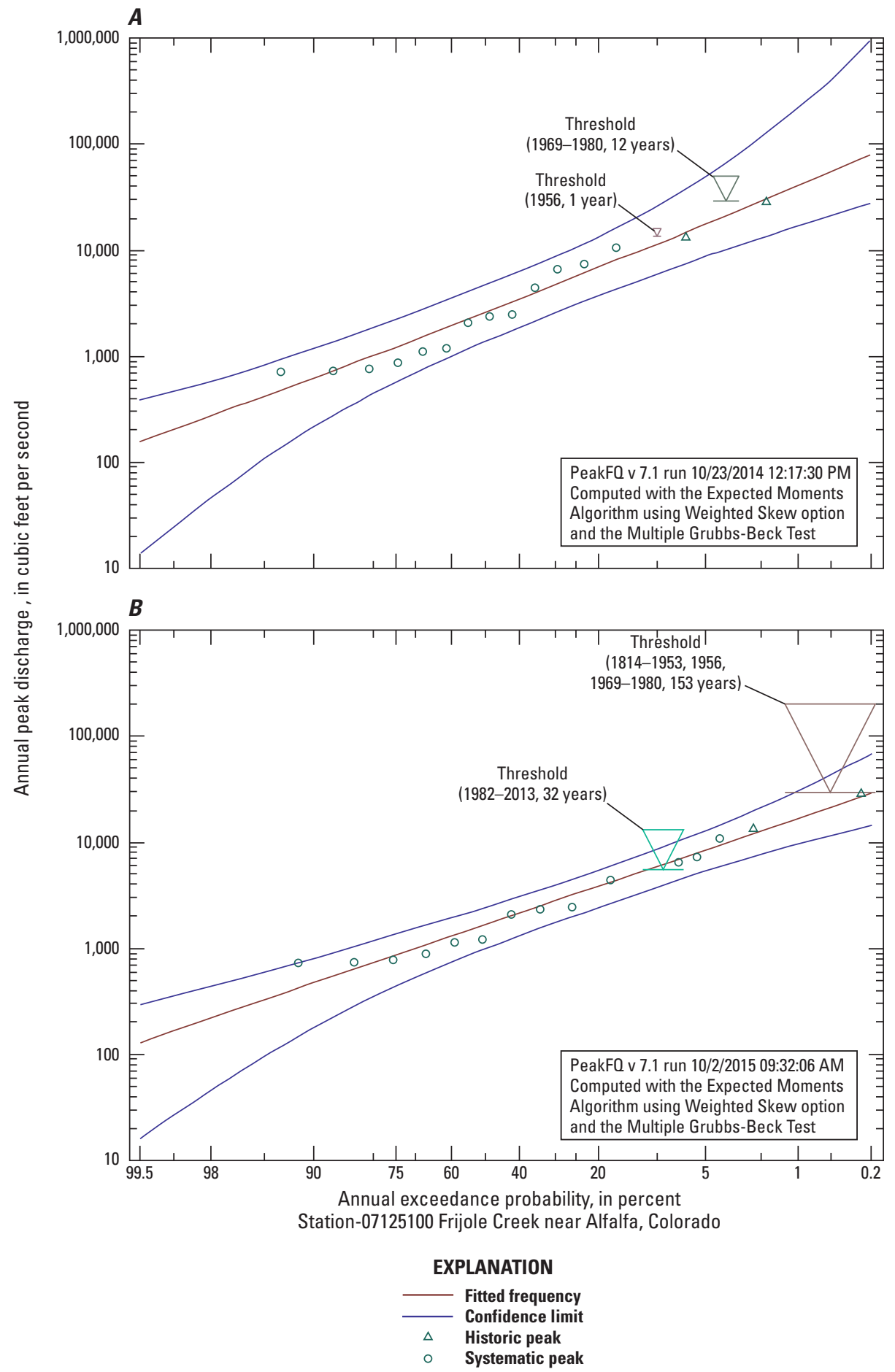

Figure 3. Flood-frequency analyses for 07125100 Frijole Creek near Alfalfa, Colo., using the $A$, 13-year streamgage record only and $B$, streamgage record with one paleoflood and one non-exceedance bound; the flood-frequency curve and its 95 -percent confidence limits are reduced with the addition of the paleoflood data; where threshold is the years for which the historic or paleoflood data is applicable. 
Paleoflood hydrology has been used extensively to document large magnitude floods to improve flood-frequency estimates (England and others, 2010; Harden and others, 2011). Paleoflood hydrology (Kochel and Baker, 1982) is the study of previously unrecorded floods using slackwater deposits and other paleostage indicators (fig. 4) (Baker, 1987) to reconstruct the magnitude of the flood and geomorphic, stratigraphic, and (or) botanic evidence to reconstruct the timing of the flood. Paleoflood hydrology has a long history in a wide variety of settings throughout the world (Costa, 1986; Patton, 1987; Wohl and others, 1994; Enzel and others, 1994; Maas and Macklin, 2002; Heine, 2004; Sheffer and others, 2008; Harden and others, 2011).

\section{Paleoflood Slackwater Deposits}

One widely used technique in the study of paleofloods uses fine-grained sedimentology records that accumulate in backwater areas (slackwater) to construct a history of past floods (Patton and others, 1979; Kochel and Baker, 1988). This approach has been developed during the last several decades and is now a widely used method for quantifying unrecorded floods (Baker, 1987; Ely and others, 1993; O'Connor and others, 1994; Sheffer and others, 2008; Harden and others, 2011). This technique can be extremely useful in characterizing the frequency of large floods; however, slackwater sediments often times do not represent a complete and continuous record of floods at a particular site. Paleoflood studies can also provide a long-term perspective that places historical large-magnitude floods into temporal context and assists in reconciliation of conflicting information (Boner and Stermitz, 1967; Helley and LaMarche, 1973).

\section{Other Paleostage Indic ators}

Another aspect of paleoflood hydrology is the concept of a paleohydrologic bound (non-exceedance bound/perception threshold), defined as a time interval during which a particular discharge has not been exceeded (Levish, 2002). Reconstructing the discharge and magnitude of individual paleofloods can be difficult to interpret in alluvial streams and rivers because of potentially complex stratigraphy and variable preservation (House and others, 2002). Additionally, individual paleofloods typically represent a minimum discharge value; whereas, nonexceedance bounds are a maximum value. Non-exceedance bound data are especially helpful in flood-frequency analyses because there is no constraint on the flood-frequency extrapolation at low probabilities using paleofloods alone (Levish, 2002). Considering these limitations, the concept of the non-exceedance bound was developed by the Bureau of

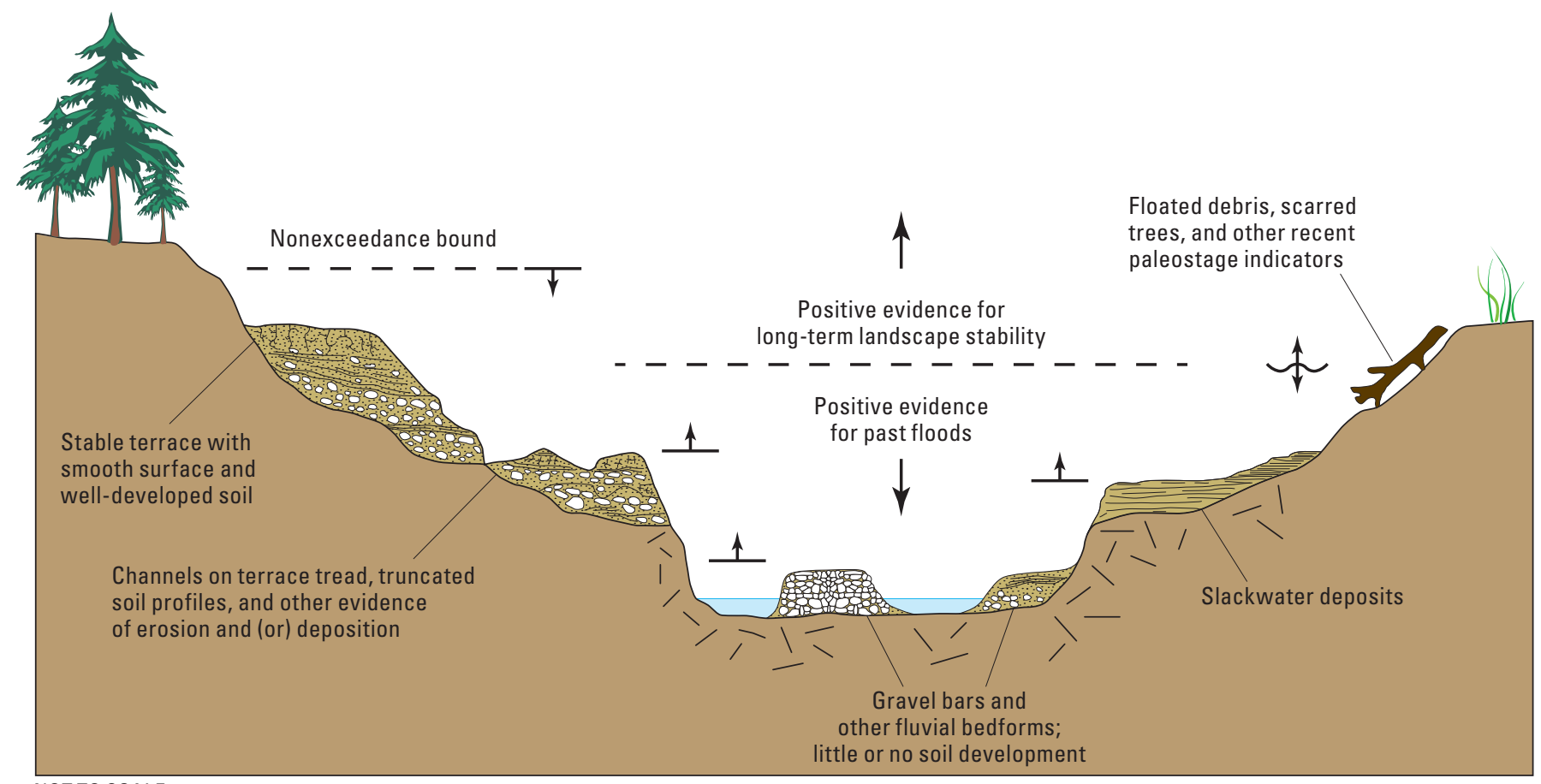

NOT TO SCALE

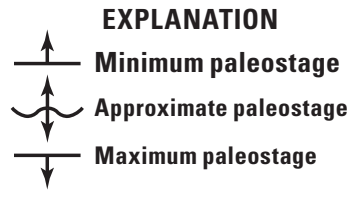

Figure 4. Idealized channel cross section illustrating the concept of a non-exceedance bound and the fluvial landforms and related deposits relative to paleoflood studies. Modified from England and others (2010). 
Reclamation in the mid-1990s because the use of paleofloods alone was not adequate for quantitative risk assessments for dam safety (Levish and others, 1994).

The non-exceedance bound methodology uses the age of a terrace surface that lacks clear evidence of recent inundation, erosion, and deposition, or alternatively shows evidence for long-term stability, to establish an upper limit to flooding. This non-inundation approach can be extremely useful in flood hazard assessment (O'Connell and others, 2002) because geomorphic and stratigraphic information derived from the terrace surface can provide an upper limit or bound on the age and magnitude of extreme floods (Levish, 2002). Rather than constructing a detailed record of past floods, the non-inundation approach focuses on identifying a non-exceedance bound.

Establishing a non-exceedance bound is accomplished by identifying terrace surfaces that serve as limits for the paleostage of large floods and estimating ages for those terraces (fig. 4). These bounds do not represent actual floods, but instead physical limits to peak flood stage over a measured time interval. Simply stated, a non-exceedance bound is a maximum stage that has not been exceeded in the time period since the terrace surface stabilized. The maximum stage can be used to estimate peak discharge given some knowledge of the channel characteristics. Developing evidence of specific paleofloods using this methodology is not necessary; however, determining the discharge for a flood that has not been exceeded over the time interval represented by the preserved stable landscape is important. Early studies by Mansfield (1938) on the Ohio River and Jahns (1947) on the Connecticut River recognized the concept of these noninundation bounds. Mansfield (1938) and Jahns (1947) found that floods on those rivers overtopped stream terraces that had not been inundated for thousands of years.

\section{Methodology Used to Identify Paleofloods and Respective Ages}

An effective means of reducing uncertainties regarding probabilities of flood recurrence is to augment observational and historic peak streamflow records by applying paleoflood techniques (Costa, 1978; Stedinger and Baker, 1987; Frances and others, 1994; Webb and others, 2002) which typically use geologic, geomorphic, and paleobotanical evidence to determine the ages and magnitudes of floods that occurred before the collection of observational records.

For this study, the primary evidence for past floods consists of fine-grained deposits found on terraces and debris/ flotsam lines found along the channel margin (fig. $5 \mathrm{~A}$ ). The stratigraphic record present along streams in the form of terrace and flood-plain deposits can be direct indicators of the magnitude of large floods on a river and may be 10- to 100-times longer than conventional streamgage records of large floods (Patton, 1987; Baker, 1989; Jarrett, 1991).

When a large flood occurs, sediment is transported in the flood waters and deposited on terrace surfaces, channel bars, or in slackwater settings, such as in the lee of bedrock obstructions or in the mouths of tributary junctions. A distinct record of the flood is preserved in the stratigraphy of the terrace (fig. 5B-D). The deposits left by more recent floods will show sedimentologic characteristics of a fluvial deposit such as visible cross bedding, laminations, or other bedding features but no soil development (fig. 5C). Over time, the flood deposit will begin to form a soil and develop soil structure and horizons. Evidence for multiple floods can be detected in the stratigraphic record by the identification of stratigraphic breaks (fig. $5 D$ ), such as buried soils, organic mats, colluvium (hillslope deposits), tree rooting-horizons, and burn horizons.

Other evidence of unrecorded floods at the study sites was the presence of high-water marks such as flotsam and trimlines (erosional features caused by a flood) along the channel margins (fig. $5 A$ and $B$ ). Although most of the floods associated with the high-water marks occurred within the last few decades, these floods were not captured in the streamgage record because of the prevalence of discontinued streamgages in the study area. Many of the drainage basins investigated had lines of fluvially transported debris such as logs, branches, leaves, pine needles, bark, and twigs that were above the stage of the peak of record for measured (known as systematic peaks in this report) discharges in the streamgage records. These lines clearly indicate (1) the occurrence of a large flood, and (2) the stage of the flood. An estimate of age can be determined from the degree of weathering of the debris. For example, logs and large branches can persist in dry environments for more than 100 years but small twigs, pine needles, and leaves last only a few years. Therefore, in drainage basins where large floods were measured from debris lines, a young age was assigned if the organic fines were still visible, but an older age if only the large debris remained. Any historic artifacts found in flotsam or flood deposits can be used to estimate the age of the flood as well (House and Baker, 2001). An example of paleoflood evidence from 06758700 Middle Bijou Creek Tributary near Deer Trail, Colo., is given in Appendix 1.

\section{Methodology Used to Identify Non-Exceedance Bounds and Respective Ages}

Evidence used to determine non-exceedance bounds for this study consisted of trimlines, soil characteristics, desert pavement (layer of flat-lying, densely-packed rocks, pebbles, or gravel overtopping a vesicular silty layer that can develop on the ground surface in arid environments), physical weathering of rocks and terraces, and vegetation (fig. 6). Trimlines, erosional features caused by a flood and typically located along the channel margin, can serve as a stage estimate for a non-exceedance bound if no other evidence of flooding is observed above the trimline (fig. $6 A$ ). This typically is used at locations with a clear trimline and a lack of terraces. Age determination typically depends on the degree of soil development above the trimline, similar to determining the age of terraces described in the following three praragraphs.

Soils formed on alluvium in stream terraces are a commonly used method to identify and age non-exceedance bounds (Levish and Ostenaa, 1996; Jacoby and others, 2007; England 


\section{A. Little Fountain Creek near Fountain, Colorado, 07105940}

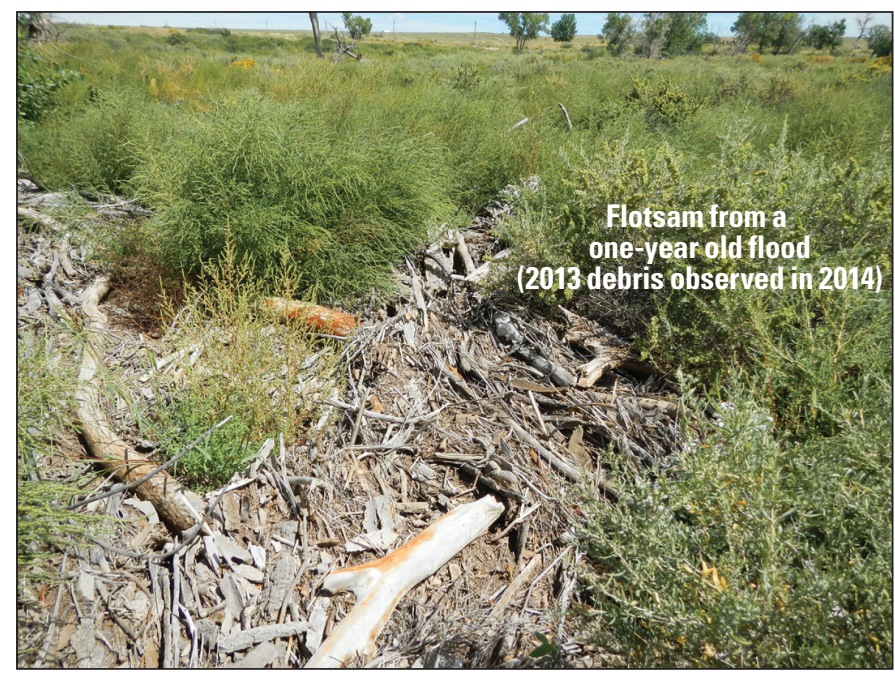

\section{Arikaree River at Haigler, Nebraska, 06821500}

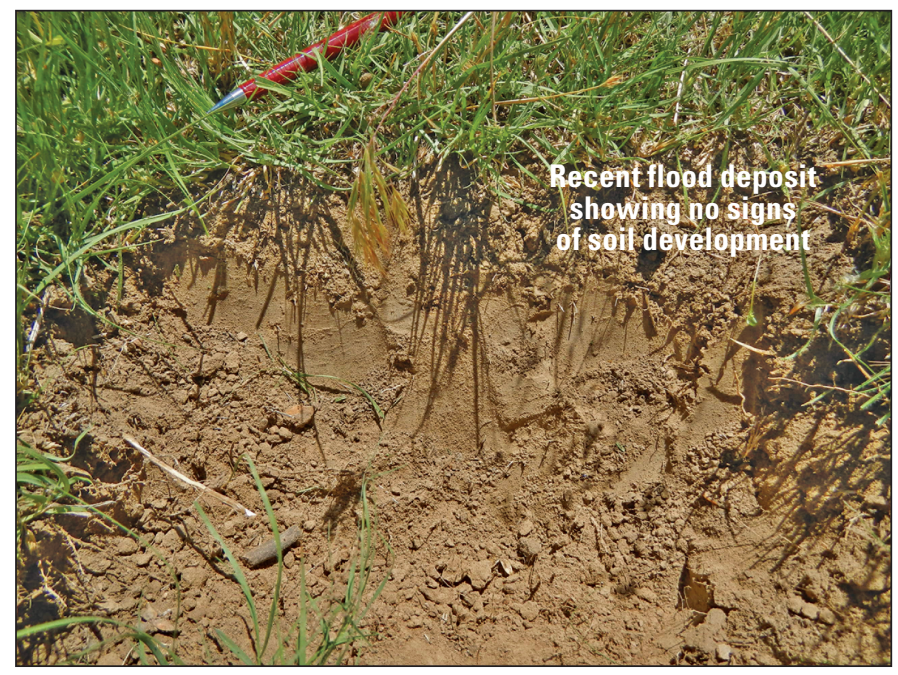

\section{B. Frijole Creek near Alfalfa, Colorado, 07125100}

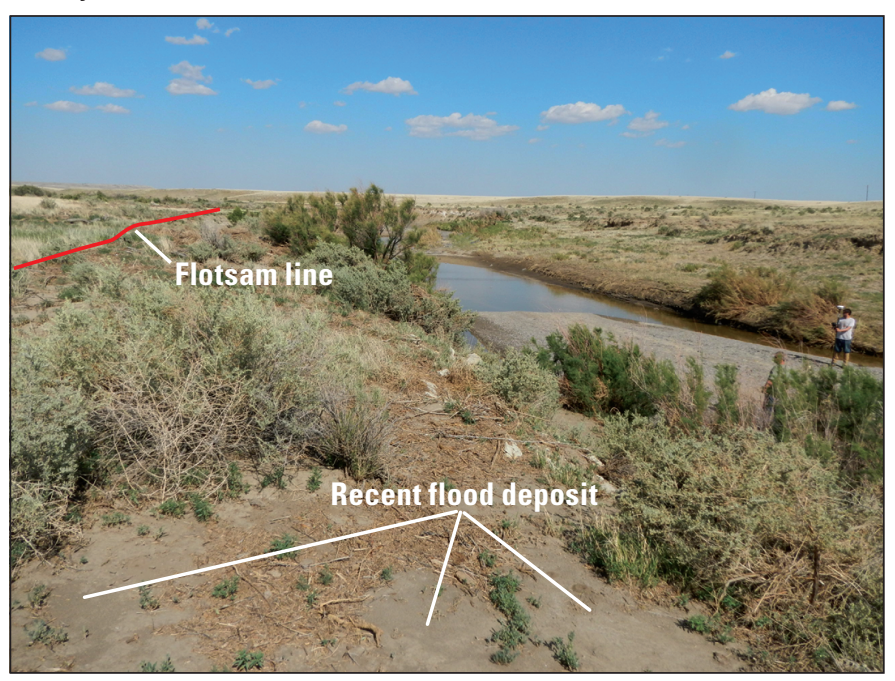

\section{Kettle Creek above U.S. Air Force Academy, Colorado, 07103960}

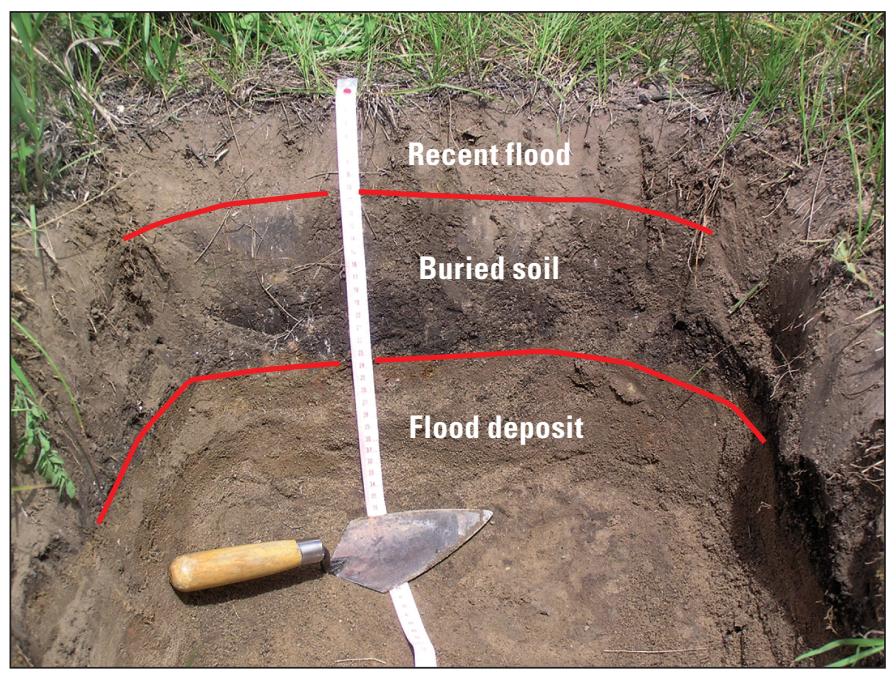

Figure 5. Evidence of flooding at $A, 07105940$ Little Fountain Creek near Fountain, Colo., $B, 07125100$ Frijole Creek near Alfalfa, Colo., $C, 06821500$ Arikaree River at Haigler, Nebr., and D, 07103960 Kettle Creek above U.S. Air Force Academy, Colo., $A$ through $D$ all show evidence of recent flooding; however, because many of the streamgages in the study area were discontinued, these floods were unrecorded, $D$, shows evidence of an older flood as indicated by the buried soil. Photographs by Tessa Harden, USGS ( $A-C)$ and Jeanne Godaire, Bureau of Reclamation (D).

and others, 2010). Stable terrace surfaces are simply flood plains that have been abandoned because of either stream incision and (or) channel migration. Once abandoned, the surface characteristics of the terrace begin to change recognizably with time. An abandoned terrace surface will tend to lose all evidence of having been inundated and become more planar and smooth with time. Once stabilized, soil will begin to form on the terrace deposits (fig. 6B). Thus, stable terrace surfaces represent the field expression for the level of non-inundation or nonexceedance bounds and are a direct indication for the physical upper limit of floods along a stream through time.

The rate of soil development is dependent on several factors as first described by Jenny (1941), the most important of which include climate, organisms, relief, parent material, and time. The development of soil properties is related to these factors and can be used to estimate the age of a particular soil, and thus the age since the terrace surface stabilized. Where multiple fluvial landforms or landforms formed by other processes are present, a soil chronosequence can be developed. A soil chronosequence is defined as, "A sequence of related soils that differ in their degree of profile development because of differences in their age" (Allaby, 1998). Soil chronosequences using properties of soils and absolute or relative age dating have been developed in various regions and can be used at many of the study locations for this study to estimate the age of a soil based on these time-dependent soil properties (Birkeland, 1999). The most important soil properties that are used for correlation in this study include the presence or 
A. Lockwood Canyon Creek near Thatcher, Colorado, 07126390

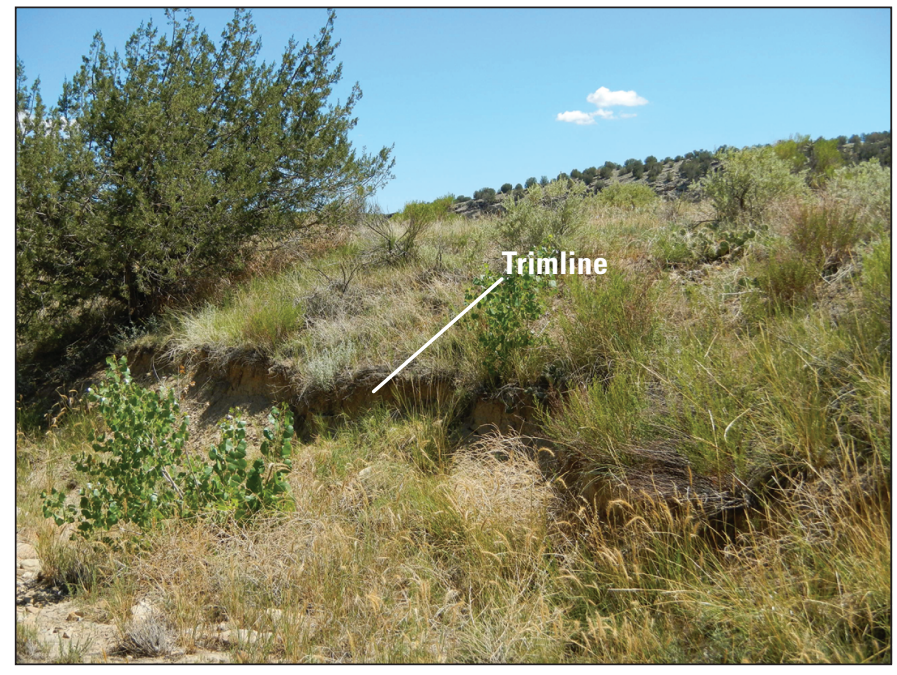

\section{Frijole Creek near Alfalfa, Colorado, 07125100}

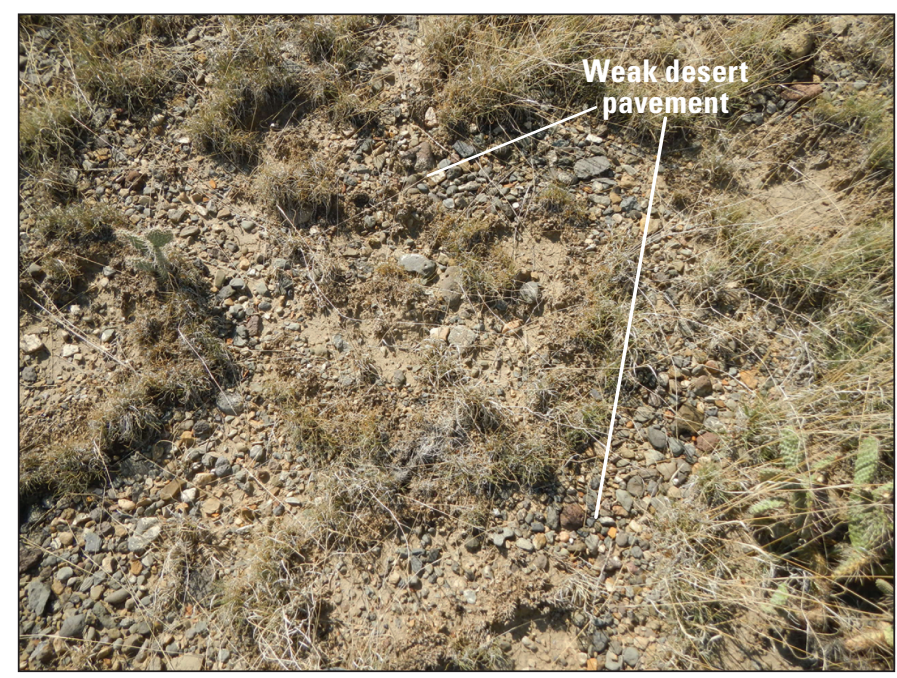

\section{B. Big Arroyo near Thatcher, Colorado, 07120620}

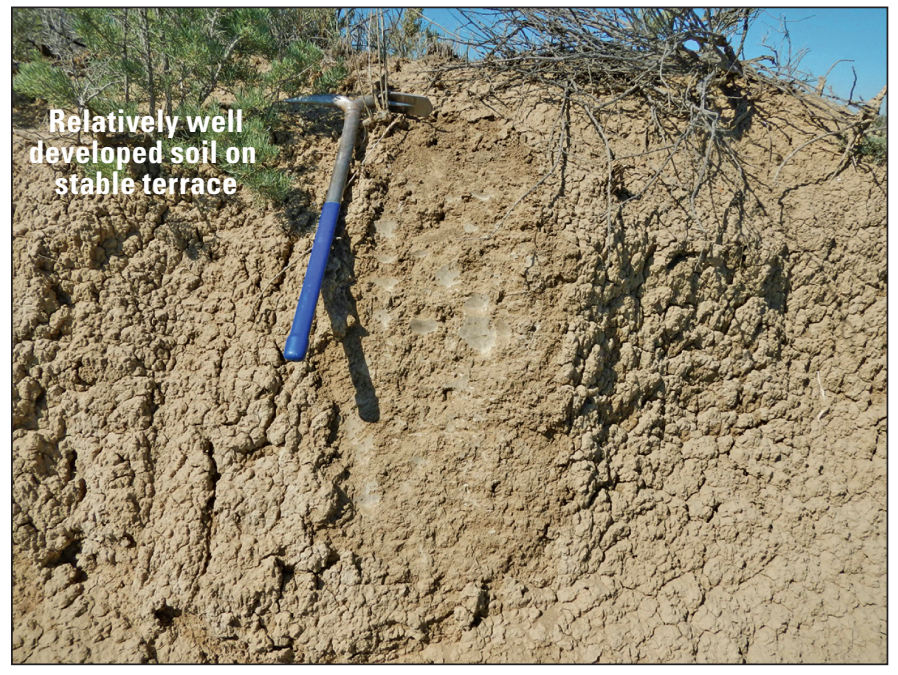

D. Frijole Creek near Alfalfa, Colorado, 07125100

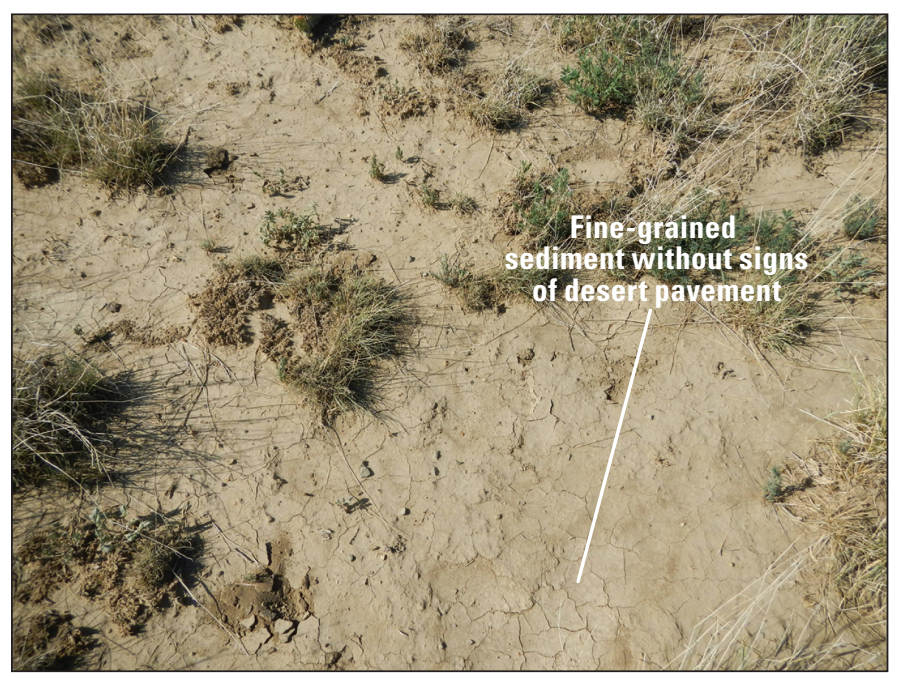

Figure 6. Evidence used to determine non-exceedance bounds. $A$, trimline at 07126390 Lockwood Canyon Creek near Thatcher, Colo., $B$, well developed, stable soil at 07120620 Big Arroyo near Thatcher, Colo., $C$, weak desert pavement at 07125100 Frijole Creek near Alfalfa, Colo., $D$, also at 07125100 Frijole Creek near Alfalfa, Colo., no desert pavement development indicating a recently disturbed surface. Photographs by Tessa Harden, USGS $(A-D)$.

thickness of organic-rich A horizons, carbonate accumulation, and color. In addition, Bureau of Reclamation and other researchers have conducted paleoflood, geomorphic, and geologic investigations in the region of this study; radiocarbon data and soil properties from these previous studies were used to estimate the age of deposits for this study by correlating terrace surfaces and their associated soil properties (Hunt, 1954; Scott, 1960; Scott, 1963; Machette, 1985; Cornwell, 1986; May, 2003).

The ages associated with the terrace surfaces that form the non-exceedance bounds should always be considered minimum ages because of the problems related to determining the precise time when a particular surface was abandoned, stabilized, and soil began forming. Determining the age of soils can be complicated because the soils contain a wide variety of organic components because of the relatively continual input of new material introduced into the profile by biotic processes (Johnson, 1990; Hseih, 1992, 1993; Birkeland, 1999; Puseman and Cummings, 2004). Therefore, an estimate for the maximum discharge over the minimum time interval since the terrace surface stabilized makes the non-exceedance bound a conservative estimate for the flood hazard. These estimates are made even more conservative with time because channels may down cut and erode laterally resulting in apparently larger cross sections and peak discharges than those that may have actually been present or occurred, so underestimation of the peak discharge associated with a non-exceedance bound in most cases is typically not a severe problem for floodfrequency analysis given the other uncertainties inherent in the method. 
The use of desert pavement was also used to determine age and stage of non-exceedance bounds on appropriate streams. Desert pavement is a layer of flat-lying, denselypacked rocks, pebbles, or gravel overtopping a vesicular silty layer that can develop on the ground surface in arid environments (fig. 6C). Over time, a varnish or patina forms on the rocks. There is much debate on the processes that drive the formation of desert pavement (Jessup, 1960; Cook, 1970; McFadden and others, 1987; Williams and Zimbelman, 1994). However, it has been well documented that the presence of the pavement indicates the area has not been disturbed for a period of time depending on the degree of development (fig. 6C and $D$ ) and can be used as a relative indicator of age (Quade, 2001; Anderson and others, 2002; Pelletier and others, 2007).
Well-developed pavements can form on time scales of hundreds to thousands of years or longer (McFadden and others, 1998; Quade, 2001; Pelletier and others, 2007).

The relative degree of weathering of rocks on the surface of terraces can also be used as an indicator of the age and stage of non-exceedance bounds. Floods will typically mobilize sediment on the surface and scour larger rocks that are not mobilized. Rock tumbling and scour of large rocks by other rocks or debris in the flood will remove weathered surfaces and thus expose fresh faces with irregular or weak weathering (fig. 7A), thus resetting the weathering "clock" on the surface of the clast. Conversely, terrace surfaces that have not been inundated for a long period of time will start to develop rills and other small scale surface drainage features. Lichen growing on the top of rocks on terrace surfaces also indicates

\section{A. Taylor Arroyo below Rock Crossing, near Thatcher, Colorado,} 07126325

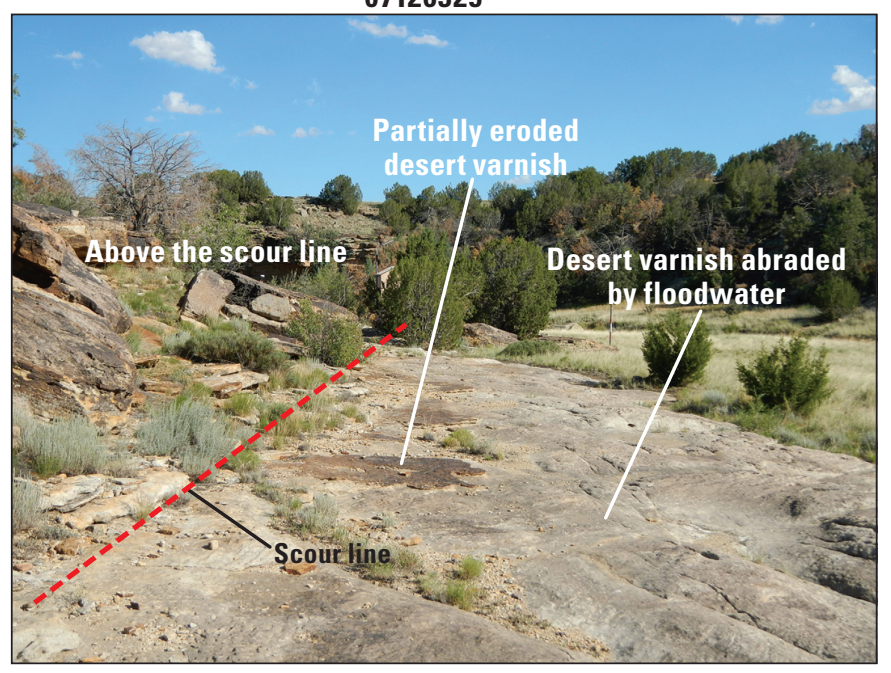

\section{Arikaree River at Haigler, Nebraska, 06821500}

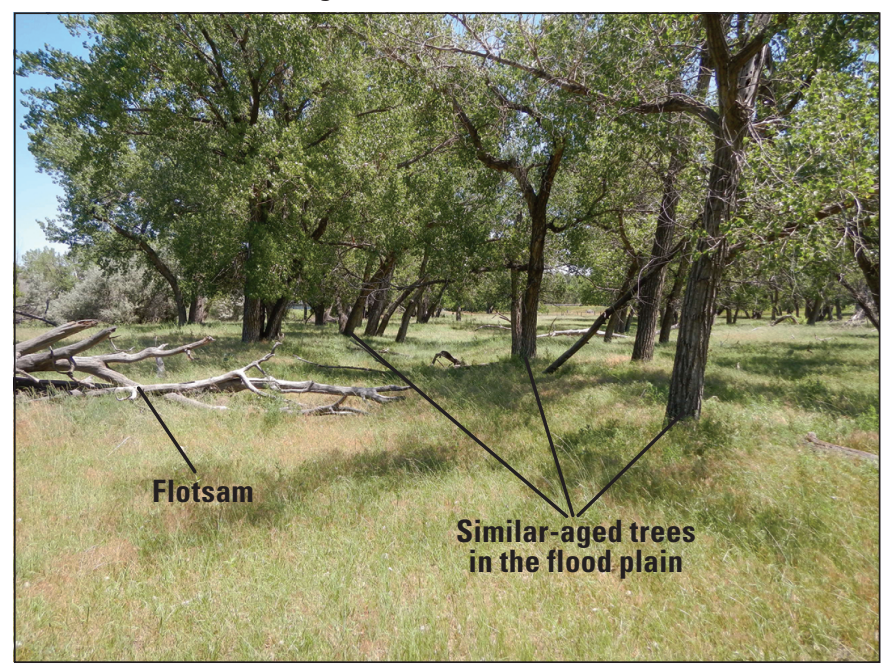

\section{B. Taylor Arroyo below Rock Crossing, near Thatcher, Colorado,} 07126325

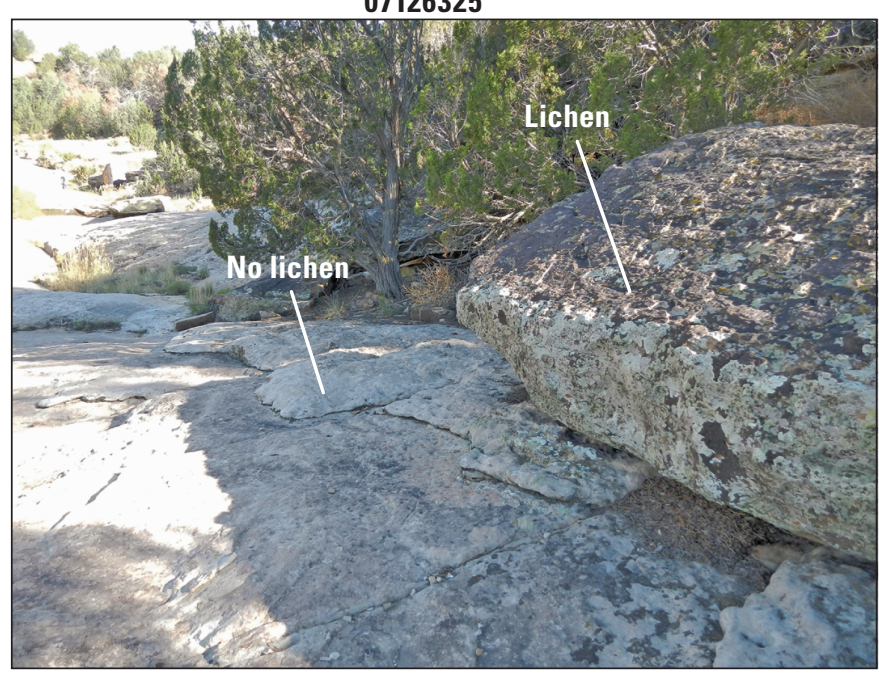

\section{Blue Creek near Lewellen, Nebraska, 06687000}

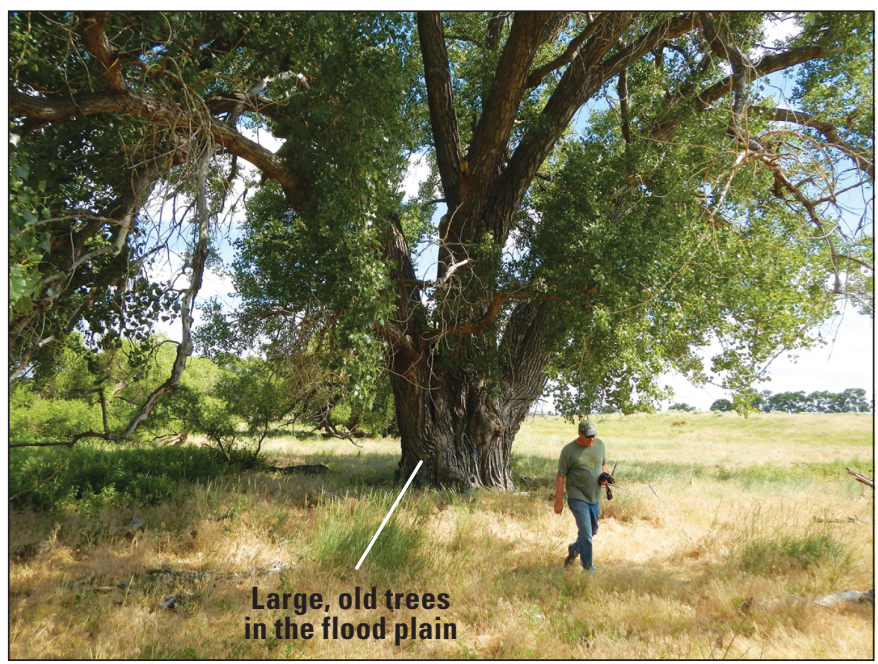

Figure 7. Evidence used to determine non-exceedance bounds. $A$, freshly scoured rock (right) and weathered rock (left) at 07126325 Taylor Arroyo below Rock Crossing, near Thatcher, Colo., $B$, lichens that have not been scoured from recent floods (top right) and freshly scoured rock (bottom left) at 07126325 Taylor Arroyo below Rock Crossing, near Thatcher, Colo., $C$, similar aged stand of cottonwood trees at 06821500 Arikaree River at Haigler, Nebr., D, old cottonwood at 06687000 Blue Creek near Lewellen, Nebr. Photographs by Tessa Harden, USGS ( $A-D$ ). 
that the sediment has not been mobilized during the growth of the lichen (fig. $7 B$ ) because these organisms would likely be scoured from rock surfaces during a flood over the terrace surface (Gregory, 1976). Although there are challenges with estimating the age of lichen for some species (Beschel, 1973; Birkeland, 1973; Osborn and others, 2015), lichen can be used along with other indicators to estimate a minimum time over which the surface has not been inundated by a large flood.

Vegetation can aid in defining a non-exceedance bound in several ways. Assuming no anthropogenic disturbance, a stand of trees of similar age located along a river on a flood bar, terrace, or other landform, could indicate the last time a large flood of a particular magnitude occurred on the river (fig. $7 \mathrm{C}$ ). This is particularly true for the ephemeral streams in the semiarid regions of the study area. Cottonwoods are the dominant riparian tree in this area and have been shown to repopulate surfaces after large floods because of the abundance of bare moist ground (Scott and others, 1997; Mahoney and Rood, 1998). The age of the large flood would be close to the age of the trees assuming trees started to regrow after the flood (Friedman and others, 1996; Lukas and Woodhouse, 2006). Conversely, old trees rooted on the surface of a terrace might indicate an absence of floods large enough to significantly overtop the surface and either partially bury the tree with flood sediment or uproot the tree (fig. 7D); (Scott and others, 1997). East of Denver (fig. 1), in eastern Colorado, Friedman and Lee (2002) documented sites that have not experienced large floods in the last 80 years that have limited cottonwood production, a low tree density, and more abundant grassland than sites that have experienced a more recent large flood. The age of trees was determined in this study by using previously developed relations between tree diameter and growth rates published by the International Society of Arboriculture (Missouri Department of Conservation, 2015; Nix, 2015).

Paleoflood and non-exceedance bound ages were established based on reconnaissance-level methods as discussed previously. Multiple lines of evidence were used at each streamgage to determine the age estimate to add a higher degree of certainty to reconnaissance-level estimates. Absolute ages, such as from radiocarbon dating, were not obtained for any of the streamgages. However, because the focus of this study was on floods with annual-exceedance probabilities (AEPs) of 0.2 percent and greater, an area of the calibration curve where radiocarbon dating can have low resolution (Stuvier and Becker, 1986; Stuvier and Pearson, 1986), age estimates using a combination of geomorphic characteristics, vegetation, historical artifacts, and soil properties are credible and can provide comparable age range estimates to those obtained by radiocarbon dating. Paleoflood or non-exceedance bound evidence was documented at 41 streamgages and 3 streamgages had previously collected paleoflood data (Jarrett, 2000; England and others, 2003) for a total of 44 streamgages with paleoflood or non-exceedance bound evidence to be used to compute the AEPDs.

\section{Computing Discharge of Paleofloods}

To determine the peak discharge of a paleoflood or nonexceedance bound, two different hydraulic models were used. A hydraulic model is used to predict streamflow characteristics including water-surface elevation of a free-flowing channel given a particular discharge or discharge-time series. Models were constructed using cross sections, paleoflood stages, channel slope, and any existing high-water marks surveyed using survey-grade Global Navigation Satellite Systems following the methods described in Rydlund and Densmore (2012).

\section{Hydrologic Engineering Center River Analysis System Computation}

For streamgages where only two cross sections were surveyed, the U.S. Army Corps of Engineers Hydrologic Engineering Center River Analysis System (HEC-RAS) version 4.1 was used to simulate streamflow. HEC-RAS is applicable to a wide range of scenarios, including large networks of regulated or natural streams or rivers to constructed channels (U.S. Army Corps of Engineers, 2010a). HEC-RAS is a one-dimensional depth-averaged hydraulic model capable of computing water-surface elevations and horizontal-velocity components for subcritical and supercritical free-surface flow regimes (U.S. Army Corps of Engineers, 2010b). The model is designed for problems in which vertical accelerations are negligible, and velocity vectors generally point in the same direction over the entire depth of the water column at any discrete period in time (U.S. Army Corps of Engineers, 2010b). The Manning roughness coefficients determined in the field using the Cowan method (Cowan, 1956) and subdivided into three sections (left bank, channel, and right bank) were included in the models. The HEC-RAS models were configured with discharge as the upstream boundary condition and the channel slope was used for the normal depth downstream boundary condition. The channel slope was either surveyed in the field from channel invert elevations or high-water mark profiles or was determined from a 1:24,000 scale topographic map. Iterations of various discharges were modeled using HEC-RAS until the water-surface profile matched the stage of the paleoflood identified in the field. The corresponding discharge was then used for the paleoflood or non-exceedance bound.

\section{Slope-Area Computation}

For streamgages where three or more cross sections were surveyed, the USGS Slope-Area Computation Graphical User Interface (SACGUI) program was used (Bradley, 2012). In the slope-area method, discharge is computed on the basis of a uniform-flow equation involving channel characteristics, water-surface profiles, and a roughness or retardation coefficient (Dalrymple and Benson, 1967). The slope-area method is based on one-dimensional gradually varied, steady-flow equations and uses the conservation of energy and mass and the normal-flow equation to determine discharge (Fulford, 
1994). The SACGUI program uses a high-water mark profile which was determined from the channel slope. The stage of the high-water marks was adjusted while keeping the slope constant until the water-surface profile matched the stage of the paleoflood. Resulting discharge values were used for the paleoflood or non-exceedance bound estimate. An example computation from SACGUI for 06758700 Middle Bijou Creek Tributary near Deer Trail, Colo. is located in Appendix 1.

\section{Model Uncertainty}

Estimates of discharge uncertainty for paleofloods and non-exceedance bounds can be used by the USGS software program PeakFQ (Veilleux and others, 2014) to produce more accurate flood-frequency analysis by using the upper and lower limits of the uncertainty in the paleodischarge estimate. The lower limit discharge was computed by using the lowest stage of the paleoflood or non-exceedance bound evidence, increasing the Manning roughness coefficient by 20 percent, and decreasing the channel slope by 25 percent. The upper limit discharge was computed by using the highest stage of the paleoflood or non-exceedance bound plus 1 foot ( 3 feet for non-exceedance bound evidence), decreasing the Manning roughness coefficient by 20 percent, and increasing the channel slope by 25 percent. These uncertainty thresholds were chosen because the thresholds fully encompass the range of reasonable values that could potentially have occurred. The type, discharge, age, uncertainties, and computation method of the paleoflood data for all 44 streamgages where paleoflood information was used to develop the PSRREs for peakstreamflow are located in Appendix 2.

\section{Flood-Frequency Analysis}

The annual series of peak-streamflow data at 188 continuous-record streamgages and crest-stage gages were used to estimate AEPDs, such as the 100-year flood discharge. AEPDs from streamgage data provide the basis for developing PSRREs. The estimated AEPDs for each streamgage were computed using the USGS software program PeakFQ version 7.1 (Veilleux and others, 2014) for annual-peak streamflows. The AEPDs were determined using systematic data through water year 2013 (October 1, 2012, through September 30, 2013). This report expresses flood-frequency estimates in terms of AEPs, which are the reciprocals of the recurrence intervals. AEP can also be represented in percent, and a particular flood-frequency estimate is then termed the " $P$-percent chance discharge," where $P$ is the probability, in percent, that the discharge will be equaled or exceeded in any year. For example, a 10-year flood discharge is the same as a discharge having a $0.10 \mathrm{AEP}$; this flood discharge also is described as a 10-percent flood or $Q_{10 \%}$ (Southard and Veilleux, 2014).

For this report, the log-Pearson Type III frequency distribution was fit to the logarithms of the annual-peak flows to determine flood-frequency estimates following the guidelines established in Bulletin 17B (Interagency Advisory Committee on Water Data, 1982). The mean, standard deviation, and skew coefficients were used to fit the distribution to describe the mid-point, slope, and curvature of the flood-frequency curve, respectively (Gotvald and others, 2012). Estimates of the $P$-percent AEPDs for each streamgage are computed by inserting the three statistics of the frequency distribution into the equation:

$$
\log Q_{p}=\bar{X}+K_{p} S
$$

where

$Q_{p} \quad$ is the $P$-percent annual exceedanceprobability discharge, in cubic feet per second;

$\bar{X} \quad$ is the mean of the base 10 logarithms of the annual peak discharges;

$K_{p} \quad$ is a factor based on the skew coefficient and the given percent $\mathrm{AEP}$ and is obtained from Appendix 3 in Bulletin 17B (Interagency Advisory Committee on Water Data, 1982); and

$S \quad$ is the standard deviation of the logarithms of the annual peak discharges, which is a measure of the degree of variation of the annual values about the mean value.

The skew coefficient is a measure of the asymmetry of the frequency distribution and is greatly affected by the presence of high or low outliers (annual peaks that are substantially higher or lower than the trend of the data). Large positive skews typically are the result of high outliers, and large negative skews typically are the result of low outliers (Southard and Veilleux, 2014).

\section{Skew Analysis}

The skew coefficient for a streamgage is sensitive to outliers. Therefore, the station skew coefficient for streamgages with short records, common in Colorado, may not provide an accurate estimate of the data or true skew coefficient (Gotvald and others, 2009; Feaster and others, 2009; Weaver and others, 2009). Bulletin 17B (Interagency Advisory Committee on Water Data, 1982) guidelines fit a Pearson Type III distribution to the product moments (mean, standard deviation, and skew) of the logarithms of annual peak streamflow. To compensate for effects of short record at a station, station skew is combined or weighted with a generalized value that is derived from a generalized or weighted skew map, which is included in Bulletin 17B (Interagency Advisory Committee on Water Data, 1982). The weighted skew used in the analysis for this report was determined by weighting the streamgage skew and the regional skew and is inversely proportional to their respective mean square errors, as shown in equation 5 of Bulletin 17B (Interagency Advisory Committee on Water Data, 1982): 


$$
G_{w}=\left[M S E_{G_{R}}\left(G_{s}\right)+M S E_{G_{S}}\left(G_{R}\right)\right] /\left(M S E_{G_{R}}+M S E_{G_{S}}\right)
$$

where

$$
\begin{array}{ll}
G_{w} & \text { is the weighted skew, } \\
G_{S} & \text { is the station skew, } \\
G_{R} & \text { is the regional skew, and }
\end{array}
$$

$M S E_{G_{R}}$ and $M S E_{G_{S}}$ are the mean square errors of the regional and station skew, respectively.

The generalized or regional skew used in this report was from the national generalized skew map (plate I, Bulletin 17B [Interagency Advisory Committee on Water Data, 1982]) based on streamgage data through water year 1973, which is the most current skew map in Colorado. Additional information on skew can be found in Bulletin 17B (Interagency Advisory Committee on Water Data, 1982) and in Eash and others (2013).

\section{Expected Moments Algorithm}

In this study, the Expected Moments Algorithm (EMA) with the multiple Grubbs-Beck (MGB) test method (Grubbs and Beck, 1972) was used to compute Log-Pearson Type III exceedance-probability estimates for all 188 streamgages evaluated to develop PSRREs for eastern Colorado. The USGS software program PeakFQ version 7.1 (Veilleux and others, 2014) automates the EMA/MGB procedure described in this section of the report.

The EMA retains the essential structure and momentsbased approach of the existing Bulletin 17B (Interagency Advisory Committee on Water Data, 1982) procedures to determine flood frequency but addresses several concerns about the methods used in Bulletin 17B. The EMA can accommodate interval data, simplifying the analysis of datasets containing censored observations, historic data, low outliers, and data points with high and low uncertainties common in paleofloods, while also providing enhanced confidence intervals for the AEPDs. Unlike Bulletin 17B (Interagency Advisory Committee on Water Data, 1982), which recognizes only two types of data: (1) systematic (annual streamflow peaks observed during systematic streamgage record) and (2) historic (streamflow peaks observed outside the streamgage record), the EMA employs a more general description of the historical period (the length of time that includes both systematic and historic peaks). This is accomplished through flow intervals to describe the peak streamflow in each year and perception thresholds to describe the range of measurable potential discharges in each year. Non-exceedance bounds, which were discussed in detail in the "Other Paleostage Indicators" section, are equivalent to perception thresholds and were adjusted when non-exceedance bound data were documented at a streamgage. Additional information on the EMA can be found in Eash and others (2013) and Southard and Veilleux (2014).

\section{Multiple Grubbs-Beck Test for Detecting Potentially Influential Low Floods}

Bulletin 17B (Interagency Advisory Committee on Water Data, 1982) recommends the use of the Grubbs-Beck test (Grubbs and Beck, 1972) to statistically identify low outliers in a sample of flood data. The MGB test is a generalization of the Grubbs-Beck method that creates the standard procedure for recognizing multiple Potentially Influential Low Floods (PILFs) (Cohn and others, 2013). In flood-frequency analysis, PILFs are annual streamflow peaks that meet three criteria: (1) their magnitude is much smaller than the flood quantile of interest; (2) PILFs occur below a statistically significant break in the flood-frequency plot; and (3) PILFs have great significance or leverage on the estimated frequency of large floods (Southard and Veilleux, 2014). The MGB test screens for PILFs at each streamgage and excludes them from the floodfrequency analysis. When an observation is identified as a PILF, the value of the smallest observation in the dataset determined to not be a PILF $\left(Q_{s}\right)$ is used as the censoring threshold in the EMA analysis (Southard and Veilleux, 2014). All annual peaks smaller than this value will be treated as censored observations with flow intervals equal to $(0, Q S)$ and perception thresholds equal to $(Q s, i n f)$ (Southard and Veilleux, 2014). Identifying PILFs and recording them as censored peaks can greatly improve estimator robustness with little or no loss of efficiency (Southard and Veilleux, 2014). Thus, the use of the MGB test can improve the fit of the AEPDs, while minimizing lack-of-fit because of unimportant PILFs in an annual peak series (Cohn and others, 2013; Veilleux and others, 2014).

It is important to distinguish between low outliers and PILFs. Low outlier typically refers to one or possibly two values in a dataset that are assumed to be homogenous and that do not conform to the trend of the other observations (Southard and Veilleux, 2014). In contrast, PILFs may constitute up to one-half of the observations and are assumed to result from physical processes that are not relevant to the processes associated with large floods. Consequently, the actual magnitudes of PILFs, because PILFs reflect physical processes that are not relevant to large floods, reveal little about the upper right-hand tail of the frequency distribution representing large floods, and thus, should not have an effect when estimating the risk of large floods (Southard and Veilleux, 2014). The term "low outlier" has been replaced with the term "PILF" to more accurately describe the situation (Interagency Advisory Committee on Water Data, 2014). Additional information on the MGB test and PILFs can be found in Gotvald and others (2012), Eash and others (2013), and Southard and Veilleux (2014).

The USGS software program PeakFQ version 7.1 (Veilleux and others, 2014) was used to compute the floodfrequency estimates for streamgages presented in this report. PeakFQ automates the EMA/MGB procedure described in this section of the report. The AEPDs from all 188 streamgages used to develop the PSRREs are given in Appendix 3. All output and input files used and generated by PeakFQ for all 188 streamgages and extra runs prior to the addition of paleoflood data including the specifications file are in Appendix 4. 
For the 44 streamgages where paleoflood or nonexceedance bound data existed, the period of record, perception thresholds, and flood data were determined, and PeakFQ was run with and without the paleoflood data. The PeakFQ analyses with and without the paleoflood data were done so that when the PSRREs were developed, the effects of the paleoflood data could be identified.

In PeakFQ, when paleoflood or non-exceedance bound evidence was available at the streamgage, the beginning of the period of record (including the systematic record) was entered as the age of the evidence (in Julian years [defined as 365.25 days which has been used in western societies for centuries]) and the end of the period of record (including the systematic record) was 2013 (the last full year prior to the evidence being collected). For example, for a streamgage with a paleoflood age of 500, the period of record (including the systematic record) was 1514 to 2013 . For paleoflood and non-exceedance bound evidence, the perception threshold was changed to the lower limit discharge computed from the uncertainty in the paleoflood data to infinity. This was done because the low perception threshold cannot be larger than the lower limit discharge computed from the uncertainty in the paleoflood data because smaller floods were not capable of being perceived at the gage based on the lower limit discharge. For paleoflood evidence only, the age (in Julian years) and the low and high limits of discharge were entered in PeakFQ. An example PeakFQ analysis with and without the inclusion of paleoflood data is given in Appendix 1 for 06758700 Middle Bijou Creek Tributary near Deer Trail, Colo. All the PeakFQ input and output files for all 188 streamgages can be found in Appendix 4.

\section{Basin and Climate Characteristics}

Based on previous studies conducted in Colorado and neighboring States (Kircher and others, 1985; Soenksen and others, 1999; Vaill, 1999; Rasmussen and Perry, 2000; Miller, 2003; Waltemeyer, 2008; Capesius and Stephens, 2009; and Lewis, 2010) and on the availability of data, 72 characteristics ( 57 basin and 15 climatic characteristics) were evaluated as candidate explanatory variables in the regression analysis (table 2). The 72 characteristics consist of physical properties of the basin, precipitation, land cover, and soil characteristics. Environmental Systems Research Institute, Inc. (Esri), ArcMap 10.1 (Esri, 2014) was used to compute the basin and climate characteristics, which were used as potential explanatory variables in the PSRREs. Values for all 72 characteristics were calculated for the 188 streamgages using various Esri ArcMap 10.1 (Esri, 2014) techniques, tools, and algorithms (table 2).

Esri ArcMap 10.1 (Esri, 2014) analyses were performed to determine basin and climate characteristics that could be used in the PSRREs at each streamgage. The basin perimeter delineations were performed using the National Hydrography Dataset Plus (McKay and others, 2014) and elevation data were analyzed to produce the parameter data listed in table 2. The elevation, mean precipitation, precipitation frequency, soil, surface lithology, and land cover data were processed with the basin outlines using the National Water Quality Assessment Area-Characterization Toolset (Price, 2010) to produce the elevation-related parameters for each basin in table 2 .

\section{Regional-Regression Analyses}

Multilinear regression was used to define statistical relations between peak streamflow and 2 or more of the 72 basin and climatic characteristics from table 2. Detailed description of the principles of regional regression is available in Helsel and Hirsch (2002) or Montgomery and others (2001). The statistical tests to evaluate model performance are described in Tasker and Stedinger (1989), Eng and others (2009), and Eash and others (2013).

\section{Definition of Flood Regions}

McCain and Jarrett (1976) originally defined five unique hydrologic regions in Colorado on the basis of physiographic and climatic characteristics. These same five hydrologic regions have been used for all Colorado peak- and low-flow studies since 1976 with minor modification (Kircher and others, 1985; Vaill, 1999; Capesius and Stephens, 2009). The scope of this report was to update the PSRREs in eastern Colorado which is defined as the Plains hydrologic region in Vaill (1999). The study area was extended 100 miles into adjacent States to include more streamgages and improve statistical robustness for development of PSRREs, because the hydrology is not affected by State boundaries. After analyzing the study area for potential regional subdivisions, dividing eastern Colorado into two new individual regions at $-104^{\circ}$ longitude resulted in PSRREs with the smallest $S M E$ and $S E P$ and largest pseudo $R^{2}$. The new hydrologic region located between $-104^{\circ}$ longitude and the Kansas-Nebraska State line will still be designated the Plains hydrologic region in this report. The hydrologic region comprising the rest of eastern Colorado will be designated the Foothills hydrologic region (fig. 8). The western edge of the Foothills hydrologic region was established by Vaill (1999) and this report follows that boundary. The western edge of the Foothills hydrologic region follows the 7,500-foot (ft) contour south from the Wyoming State line to the Chaffee-Fremont County line where the hydrologic region boundary follows the Chaffee-Fremont County line south across the Arkansas River and transitions up to the 9,000-ft contour, which is followed south to the New Mexico State line. The subdivision of eastern Colorado into two hydrologic regions was determined by maximizing the $R^{2}$ and $\operatorname{adj} R^{2}$ and minimizing the standard error, Mallow's $C_{p}$, and the predicted residual sum of squares (PRESS) statistic 
Table 2. Basin and climate characteristics evaluated for use in the peak-streamflow regional-regression equations in eastern Colorado, 2015.

[NWIS, National Water Information System; USGS, U.S. Geological Survey; NHD, National Hydrography Dataset; NED, 1 meter = 3.281 feet; National Elevation Dataset; PRISM, Parameter-Elevation Regressions on Independent Slopes Model; NOAA, National Oceanic and Atmospheric Administration; TP-40, Technical Paper 40; STATSGO, State Soil Geographic; NLCD, National Land Cover Dataset]

\begin{tabular}{|c|c|c|c|}
\hline Characteristic & Unit & Dataset & Dataset source \\
\hline Longitude & decimal degrees & NWIS & USGS (2013b) \\
\hline Basin perimeter out & feet & NHD Plus & McKay and others (2014) \\
\hline Minimum basin elevation & feet & 10-meter NED & Gesch (2007) \\
\hline Maximum basin elevation & feet & 10-meter NED & Gesch (2007) \\
\hline Elevation relief & feet & 10-meter NED & Gesch (2007) \\
\hline Percentage of basin above 7,500 feet & percent & 10-meter NED & Gesch (2007) \\
\hline $\begin{array}{l}\text { Percentage of basin with slopes greater } \\
\text { than } 30 \text { percent }\end{array}$ & percent & 10-meter NED & Gesch (2007) \\
\hline Minimum basin slope & percent & 10-meter NED & Gesch (2007) \\
\hline Maximum basin slope & percent & 10-meter NED & Gesch (2007) \\
\hline Mean basin slope ${ }^{1}, \mathrm{~S}$ & percent & 10-meter NED & Gesch (2007) \\
\hline Mean precipitation in April & inches & 800-meter PRISM & Daly and others (1994) \\
\hline Mean precipitation in May & inches & 800-meter PRISM & Daly and others (1994) \\
\hline Mean precipitation in June & inches & 800-meter PRISM & Daly and others (1994) \\
\hline Mean precipitation in July & inches & 800-meter PRISM & Daly and others (1994) \\
\hline Mean precipitation in August & inches & 800-meter PRISM & Daly and others (1994) \\
\hline Mean precipitation in September & inches & 800-meter PRISM & Daly and others (1994) \\
\hline Mean precipitation in October & inches & 800-meter PRISM & Daly and others (1994) \\
\hline Mean precipitation in November & inches & 800-meter PRISM & Daly and others (1994) \\
\hline Mean precipitation in December & inches & 800-meter PRISM & Daly and others (1994) \\
\hline 6-hour, 100-year precipitation ${ }^{1}{ }_{6} P_{100}$ & inches & $\begin{array}{l}\text { NOAAAtlas } 2 ; \\
\text { NOAAAtlas } 14 ; \text { TP-40 }\end{array}$ & $\begin{array}{l}\text { Bonnin and others }(2011)^{2} \text {, Miller and others } \\
(1973)^{3} \text {, Perica and others }(2013)^{4} \text {, Hershfield } \\
(1961)^{5}\end{array}$ \\
\hline 24-hour, 100-year precipitation & inches & $\begin{array}{l}\text { NOAAAtlas } 2 ; \\
\text { NOAAAtlas } 14 ; \text { TP-40 }\end{array}$ & $\begin{array}{l}\text { Bonnin and others }(2011)^{2} \text {, Miller and others } \\
(1973)^{3} \text {, Perica and others }(2013)^{4}, \text { Hershfield } \\
(1961)^{5}\end{array}$ \\
\hline Percent of hydrologic group B soils & percent & STATSGO dataset & Wolock (1997) \\
\hline Percent of hydrologic group $\mathrm{C}$ soils & percent & STATSGO dataset & Wolock (1997) \\
\hline Percent of hydrologic group D soils & percent & STATSGO dataset & Wolock (1997) \\
\hline Percent carbonate residual material & percent & USGS Surficial Lithology & Cress and others (2010) \\
\hline Percent non-carbonate residual material & percent & USGS Surficial Lithology & Cress and others (2010) \\
\hline Percent alkaline intrusive volcanic rock & percent & USGS Surficial Lithology & Cress and others (2010) \\
\hline Percent silicic residual material & percent & USGS Surficial Lithology & Cress and others (2010) \\
\hline Percent extrusive volcanic rock & percent & USGS Surficial Lithology & Cress and others (2010) \\
\hline Percent colluvial sediment & percent & USGS Surficial Lithology & Cress and others (2010) \\
\hline Percent glacial till, clayey & percent & USGS Surficial Lithology & Cress and others (2010) \\
\hline Percent glacial till, loamy & percent & USGS Surficial Lithology & Cress and others (2010) \\
\hline Percent glacial till, coarse textured & percent & USGS Surficial Lithology & Cress and others (2010) \\
\hline Percent glacial outwash and glacial lake sediment & percent & USGS Surficial Lithology & Cress and others (2010) \\
\hline Percent glacial lake sediment, fine textured & percent & USGS Surficial Lithology & Cress and others (2010) \\
\hline Percent glacial outwash and glacial lake sediment & percent & USGS Surficial Lithology & Cress and others (2010) \\
\hline Percent hydric, peat, and muck & percent & USGS Surficial Lithology & Cress and others (2010) \\
\hline Percent eolian sediment, coarse textured & percent & USGS Surficial Lithology & Cress and others (2010) \\
\hline Percent eolian sediment, fine textured & percent & USGS Surficial Lithology & Cress and others (2010) \\
\hline
\end{tabular}


Table 2. Basin and climate characteristics evaluated for use in the peak-streamflow regional-regression equations in eastern Colorado, 2015.-Continued

[NWIS, National Water Information System; USGS, U.S. Geological Survey; NHD, National Hydrography Dataset; NED, 1 meter = 3.281 feet; National Elevation Dataset; PRISM, Parameter-Elevation Regressions on Independent Slopes Model; NOAA, National Oceanic and Atmospheric Administration; TP-40, Technical Paper 40; STATSGO, State Soil Geographic; NLCD, National Land Cover Dataset]

\begin{tabular}{|c|c|c|c|}
\hline Characteristic & Unit & Dataset & Dataset source \\
\hline Percent coastal zone sediment, coarse textured & percent & USGS Surficial Lithology & Cress and others (2010) \\
\hline Percent open water & percent & NLCD 2006 & Fry and others (2011) \\
\hline Percent perennial ice/snow & percent & NLCD 2006 & Fry and others (2011) \\
\hline Percent developed, open space & percent & NLCD 2006 & Fry and others (2011) \\
\hline Percent developed, low intensity & percent & NLCD 2006 & Fry and others (2011) \\
\hline Percent developed, high intensity & percent & NLCD 2006 & Fry and others (2011) \\
\hline Percent barren land & percent & NLCD 2006 & Fry and others (2011) \\
\hline Percent deciduous forest & percent & NLCD 2006 & Fry and others (2011) \\
\hline Percent evergreen forest & percent & NLCD 2006 & Fry and others (2011) \\
\hline Percent mixed forest & percent & NLCD 2006 & Fry and others (2011) \\
\hline Percent shrub/scrub & percent & NLCD 2006 & Fry and others (2011) \\
\hline Percent emergent herbaceous wetlands & percent & NLCD 2006 & Fry and others (2011) \\
\hline \multicolumn{4}{|l|}{${ }^{1}$ Variables used in the regional regression equations. } \\
\hline \multicolumn{4}{|l|}{${ }^{2}$ Used for New Mexico. } \\
\hline \multicolumn{4}{|l|}{${ }^{3}$ Used for Wyoming. } \\
\hline${ }^{4}$ Used for Colorado, Kansas, & & & \\
\hline Ised for Texas. & & & \\
\hline
\end{tabular}

by Ordinary-Least Squares (OLS) regression procedures. Using GLS regression procedures, it was confirmed that the new regions reduced the uncertainty in the PSRREs compared to the not-subdivided region used in previous studies (Kircher and others, 1985; Vaill, 1999; Capesius and Stephens, 2009). Prior to determining that the $-104^{\circ}$ longitude minimized the PSRRE uncertainty, maps of streamgage residuals and various other subdivisions were tested based on longitude, basin outlet elevation, mean basin elevation, maximum basin elevation, drainage area, hydrologic forcing (rainfall and snowmelt compared to predominantly rainfall), and basin (Arkansas compared to lower Missouri).

\section{Development of Peak-Streamflow Regional- Regression Equations}

The PSRREs were developed for use in estimating peak streamflows for selected AEPs of 50 to 0.2 percent at gaged and ungaged locations for basins in the Plains and Foothills hydrologic regions of eastern Colorado. OLS regression techniques were performed to select the basin and climate characteristics for use as independent variables. A linear relation between the dependent and independent variables are required for OLS regression. To satisfy this criterion, variables often are transformed, and in hydrologic analyses, typically the log-transformation is used (Southard and Veilleux, 2014). The dependent response variable is the AEPD, and the independent explanatory variables are the basin and climate characteristics that describe the AEPDs. For the current (2015) study, all variables were transformed to base 10 logarithms to increase linearity prior to the development of the PSRREs.

A constant variance in the dependent variable for the range of the independent variables, referred to as homoscedasticity, about the regression line and normality of residuals also is a criterion for OLS regression. Transformation of the AEPD, in percent, and certain other variables to base 10 logarithms can enhance the homoscedasticity of the data about the regression line (Southard and Veilleux, 2014). Linearity, homoscedasticity, and normality of residuals were examined in residual plots.

The hydrologic model used in the regression analysis in this report is of the form:

$$
Q_{P}=a A^{b} B^{c} C^{d}
$$

where

$Q_{P} \quad$ is the dependent variable, $P$-percent AEPD, in cubic feet per second;

$A, B, C \quad$ are explanatory (independent) variables; and

$a, b, c, d \quad$ are regression coefficients. 


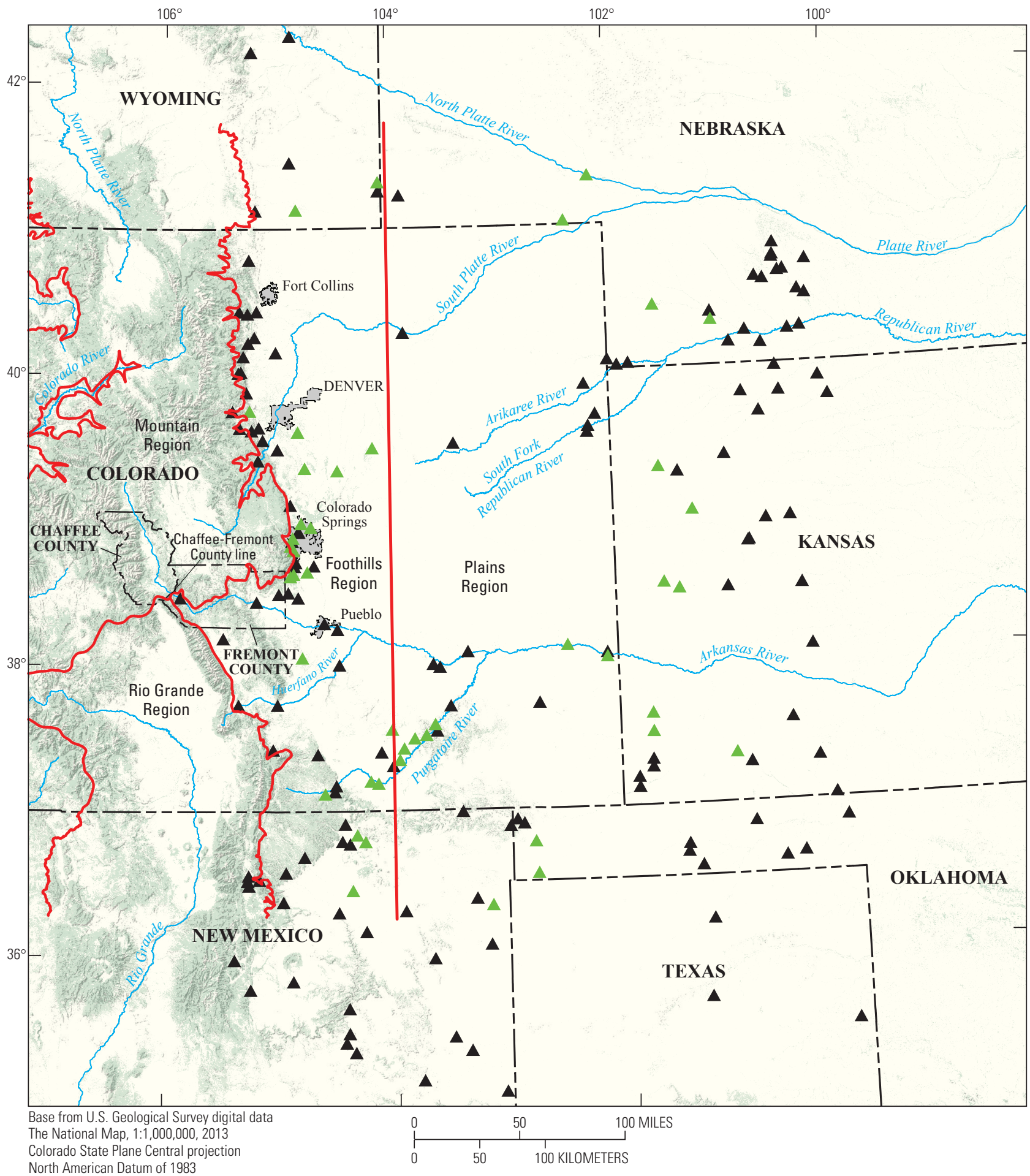

\section{EXPLANATION}

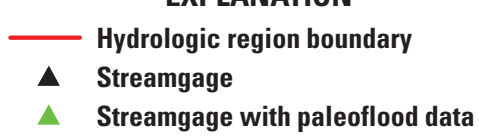

Figure 8. Boundaries of the new hydrologic regions created in eastern Colorado: the Plains hydrologic region and the Foothills hydrologic region with the location of the 99 and 89 streamgages were used to develop the peak-streamflow regional-regression equations in the Plains and Foothills hydrologic regions, respectively. 
If the dependent variable $Q_{P}$ and the independent variables $A$ and $B$ are logarithmically transformed then the hydrologic model has the following linear form:

$$
\log Q_{P}=\log a+b(\log A)+c(\log B)+d(\log C)
$$

where the variables are as previously defined. This equation is commonly written as:

$$
Q_{P}=10^{a} A^{b} B^{c} C^{d}
$$

where the variables are as previously defined.

The basin and climate characteristics (table 2) with the best correlation to peak streamflow were identified, checked for any substantial cross-correlation with other variables in the group, and were selected as the potential explanatory variables for the PSRREs. The final AEPDs for the 188 streamgages used in the regional-regression analysis are in Appendix 3.

\section{Ordinary-Least Squares Regression}

OLS regression was used in this report as an exploratory tool to limit potential explanatory variables, to a reasonable number for subsequent analysis by GLS regression, and to help determine if new hydrologic boundaries could improve the accuracy of the PSRREs. The potential explanatory variables were assessed for linear correlation with the $Q_{1 \%}$ AEPD streamflow using plots, parametric and nonparametric correlation coefficients, and the statistical significance of each explanatory variable when regressed with peak streamflow ( $p$-values), to determine the strongest predictors of peak streamflow. Transformations of equation variables were considered to determine potential for improvement of correlations with streamflow and conformance to the assumptions of linear regression application. Logarithmic transformation (base 10) of both the streamflow and the explanatory variables prior to OLS regression was found to improve normality and homoscedasticity (assumptions for parametric regression), and in most cases, substantially improve both adj $R^{2}$ and statistical significance of the slope of each explanatory variable in a regression with streamflow.

The best OLS regression models (with one-, two-, three-, and four-explanatory variables using the best of all subsets routine) for prediction of $Q_{1 \%}$ AEPD streamflow were identified and then used to limit the number of potential explanatory variables in the subsequent GLS analyses. The best PSRREs were determined by assesses the following metrics for each model: Standard Error of Estimate, $\operatorname{Adj} R^{2}$, Mallow's $C p$, and PRESS statistics. Statistical diagnostics and plots also were used to assess the regression models for meeting the assumptions of parametric regression methods. The adj $R^{2}$ statistic is maximized and the standard error of estimate, Mallow's $C p$, and PRESS statistics are minimized with accurate sets of independent variables in a regression model that explain more of the variance in the dependent variable. Additional information on OLS regression can be found in Eash and others (2013) and Southard and Veilleux (2014).

Multicollinearity (high correlation among the explanatory variables) can make results based on a multiple linear regression model misleading or erroneous, and would generally disqualify the use of both variables within a single final PSRRE. Early in the variable selection process, parametric and nonparametric correlation coefficients and plots of a particular explanatory variable with each of the other explanatory variables were used to make preliminary assessments of potential multicollinearity. When assessing candidate variables and the apparent best OLS PSRRE for further refinement by GLS regression, multicollinearity was assessed primarily by using the variance inflation factor statistic (Gotvald and others, 2012) to screen for correlated or unnecessary variables in candidate PSRREs.

\section{Generalized-Least Squares Regression}

GLS multilinear regression, as described by Stedinger and Tasker (1985), Tasker and Stedinger (1989), and Griffis and Stedinger (2007), is a method of weighting streamflow station AEPD data in the regression analysis according to differences in streamflow reliability (record lengths) and variability (record variance), and according to spatial cross correlations of concurrent streamflow among streamgages. Comparison of OLS, Weighted Least Squares (WLS), and GLS in a study by Stedinger and Tasker (1985) indicated that the weighted methods (WLS and GLS) produced results that were more accurate than the OLS regression method. When streamflow records of varying lengths or with correlated concurrent flows occurred in the dataset, the weighting technique used in GLS produces equations that are both improved in estimates of streamflow statistics and estimates of the predictive accuracy of the statistics (Stedinger and Tasker, 1985).

Based on the exploratory results from OLS regression and explanatory variable multicollinearity analysis, the computer program WREG (Eng and others, 2009) was used to compute GLS PSRREs from the best candidate variables. Five basin and climate characteristics were used as potential explanatory variables to develop the PSRREs with $Q_{1 \%}$ AEPD streamflows. The general form of the equation was defined by equation 4. Final GLS regression models were selected on the basis of minimizing values of the $S M E$ and the $S E P$, and maximizing values of pseudo $R^{2}$.

GLS models need to develop a correlation model and weighting matrix. The correlation smoothing function relates the correlation between annual-peak discharges at two streamgages to the geographic distance between the streamgages for every paired combination of the streamgages with a given number of years of concurrent streamflow. The correlation smoothing function was defined in equation 18 in Eng and others (2009). Using 30 years of concurrent streamflow, alpha of 0.001 , and theta of 0.99 , the correlation 
smoothing function was used by WREG to compute a weighting matrix for the data from 89 streamgages included in the development of the GLS PSRREs for the Foothills hydrologic region. Using 40 years of concurrent streamflow, alpha of 0.001 , and theta of 0.99 , the correlation smoothing function was used by WREG to compute a weighting matrix for the data from 99 streamgages included in the development of the GLS PSRREs for the Plains hydrologic region.

\section{Final Peak-Streamflow Regional- Regression Equations}

A total of 89 streamgages, of which 60 are in Colorado, were used to develop the PSRREs in the Foothills hydrologic region, and 99 streamgages, of which 21 are in Colorado, were used to develop the PSRREs in the Plains hydrologic region (no streamgages were used in both regions). The Foothills hydrologic region encompasses approximately $18,900 \mathrm{mi}^{2}$, and the Plains hydrologic region encompasses approximately $29,500 \mathrm{mi}^{2}$. The selection of the final basin and climatic characteristics and the evaluation of the accuracy of the PSRREs were based on the $Q_{1 \%}$ AEPD for each hydrologic region. The PSRREs from GLS regression are listed for the Foothills hydrologic region (fig. 9) and Plains hydrologic region (fig. 10).

The $S E P$, pseudo $R^{2}$, and $S M E$ for each PSRRE are reported in figures 9 and 10. The performance metrics pseudo $R^{2}$ and $S M E$ describe how well the PSRREs perform on the streamgages used in the regression analyses and the SEP measures how well the GLS regression models can predict AEPDs at ungaged sites (Eash and others, 2013). The same four explanatory variables were used to develop all eight AEPD PSRREs in the Foothills hydrologic region, and the same three explanatory variables were used to develop all eight AEPD PSRREs in the Plains hydrologic region to minimize the possibility of predictive inconsistencies between estimates of different probabilities, so that discharge estimates will increase as the discharge probability decreases. For example, maintaining the same explanatory variables helps to maximize the chance the estimate for the $Q_{1 \%}$ flood discharge is greater than the estimate for the $Q_{2 \%}$ flood discharge and the estimate for the $Q_{2 \%}$ flood discharge is greater than the estimate for the $Q_{4 \%}$ flood discharge, and so forth for all eight AEPDs in both hydrologic regions (Eash and others, 2013).

Comparisons of the number of streamgages used in the analysis and the SEP reported in the previous study (Capesius and Stephens, 2009) for the Foothills hydrologic region, and for the Plains hydrologic region are listed in table 3 . The mean $S E P$ for all 8 AEPDs in both the Foothills and Plains hydrologic regions was reduced approximately 25 percent compared to the previous study, from 147 percent to 92 percent in the Foothills hydrologic region and to 129 percent in the Plains hydrologic region (table 3 ). Additional information on SEP is presented in Olson (2014).

The four explanatory variables used in the PSRREs in the Foothills hydrologic region are drainage area in $\mathrm{mi}^{2}(A)$; 6-hour, 100-year precipitation in inches $\left({ }_{6} P_{100}\right)$; amount of soil consisting of clay in the basin in percent $(C)$; and elevation of the basin outlet in feet $\left(E_{\text {out }}\right)$. The three explanatory variables used in the PSRREs in the Plains hydrologic region are $A, C$,

Peak-Streamflow Equations for the Foothills Hydrologic Region

Generalized-least squares regression, 89 streamgages

$A$, drainage area in square miles; ${ }_{6} P_{100}, 6$-hour, 100-year precipitation; $C$, amount of clay in basin in percent; $E_{\text {out }}$, basin outlet elevation in feet; $Q_{0.5}, Q_{0.2}, Q_{0.1}, Q_{0.04}, Q_{0.02}, Q_{0.01}, Q_{0.005}, Q_{0.002}$, discharge with an annual exceedance probability of $0.5,0.2,0.1,0.04,0.02,0.01,0.005,0.002$, respectively

\section{Approximate range of predictor variables}

$A$ : 0.60-2,850 square miles, ${ }_{6} P_{100}: 2.38-4.89$ inches, $C: 9.87-37.5$ percent, and $E_{\text {out }}: 4,290-8,270$ feet

$$
\begin{array}{llllllll}
Q_{0.5} & =10^{9.952} & A^{0.626} & { }_{6} P_{100}^{1.401} & C^{0.836} & E_{\text {out }}^{-2.774} & S E P=117, & \text { pseudo } R^{2}=68, \quad S M E=111, \\
Q_{0.2}=10^{11.424} & A^{0.594} & { }_{6} P_{100}^{2.052} & C^{0.935} & E_{\text {out }}^{-3.164} & S E P=87, & \text { pseudo } R^{2}=77, \quad S M E=82, \\
Q_{0.1}=10^{12.107} & A^{0.584} & { }_{6} P_{100}^{2.372} & C^{0.974} & E_{\text {out }}^{-3.341} & S E P=80, & \text { pseudo } R^{2}=79, \quad S M E=76, \\
Q_{0.04}=10^{12.675} & A^{0.578} & { }_{6} P_{100}^{2.725} & C^{0.998} & E_{\text {out }}^{-3.486} & S E P=80, & \text { pseudo } R^{2}=80, \quad S M E=75, \\
Q_{0.02}=10^{12.977} & A^{0.575} & { }_{6} P_{100}^{2.963} & C^{1.010} & E_{\text {out }}^{-3.564} & S E P=83, & \text { pseudo } R^{2}=79, \quad S M E=79, \\
Q_{0.01}=10^{13.244} & A^{0.572} & { }_{6} P_{100}^{3.190} & C^{1.013} & E_{\text {out }}^{-3.631} & S E P=88, & \text { pseudo } R^{2}=78, \quad S M E=83, \\
Q_{0.005}=10^{13.495} & A^{0.570} & { }_{6} P_{100}^{3.386} & C^{1.024} & E_{\text {out }}^{-3.697} & S E P=94, & \text { pseudo } R^{2}=76, \quad S M E=88, \text { and } \\
Q_{0.002}=10^{13.820} & A^{0.566} & { }_{6} P_{100}^{3.621} & C^{1.038} & E_{\text {out }}^{-3.783} & S E P=104, & \text { pseudo } R^{2}=74, S M E=97 .
\end{array}
$$

Figure 9. Peak-streamflow regional-regression equations for the Foothills hydrologic region. [SEP, standard error of prediction; $S M E$, standard model error] 


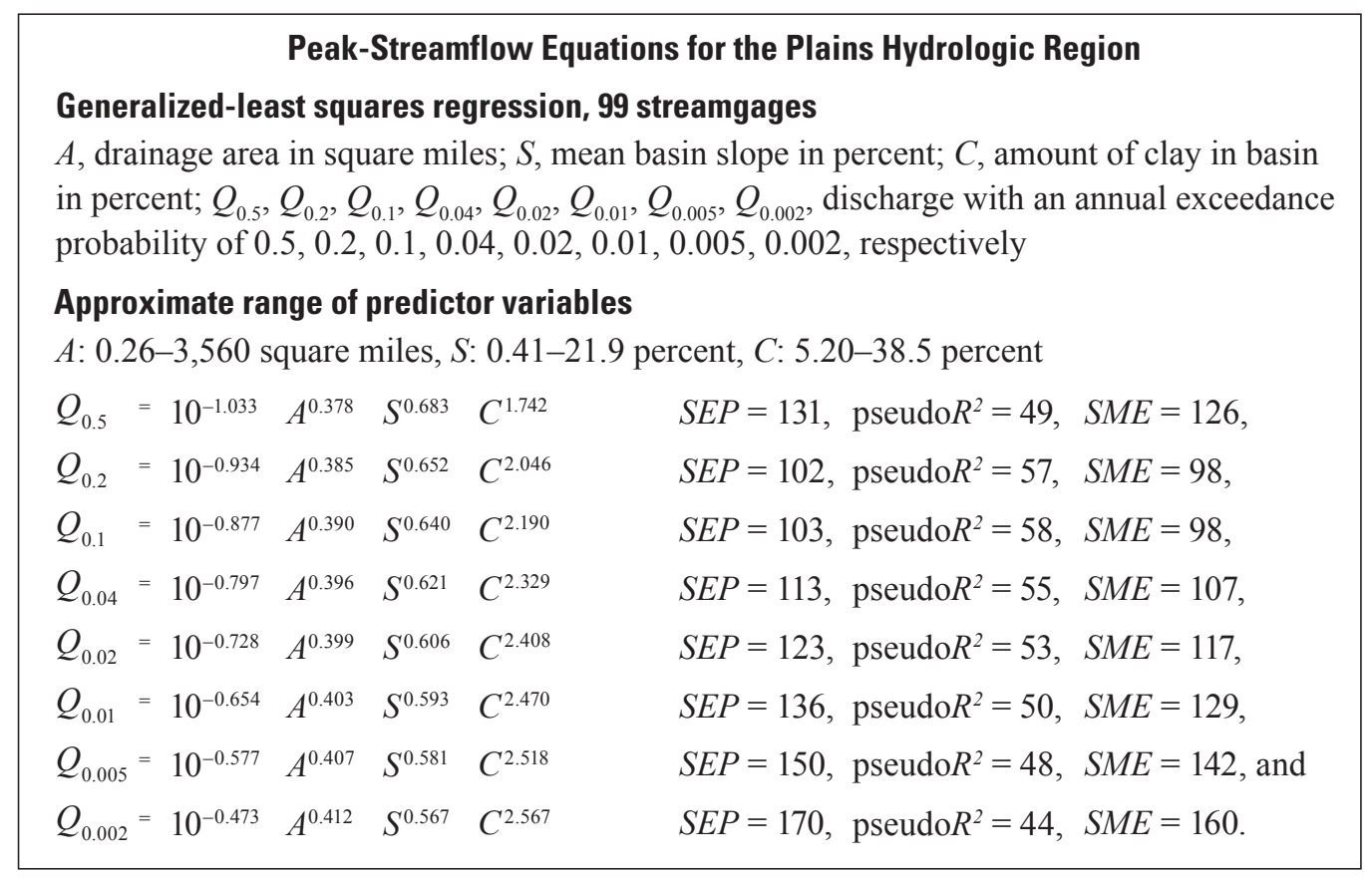

Figure 10. Peak-streamflow regional-regression equations for the Plains hydrologic region. [SEP, standard error of prediction; SME, standard model error]

and mean basin slope in percent $(S)$. As mentioned earlier, data transformations were tested for each explanatory variable used in the PSRREs and all variables were log-transformed because doing so provided the most linear relation for $Q_{1 \%}$. All explanatory variables included in the final PSRREs for both hydrologic regions were statistically significant for all AEPDs as determined by WREG and were not correlated with other explanatory variables used in the same PSRRE.

When tested independently, the most significant explanatory variable in the Foothills hydrologic region was $A$ and the second most significant explanatory variable in the Foothills hydrologic region was $E_{\text {out }}$. In the Plains hydrologic region, $A$ was also the most significant explanatory variable followed by mean basin elevation which was the second most significant explanatory variable.

In figure 11, the at-site $Q_{1 \%} \mathrm{AEPDs}$ from PeakFQ were plotted with the estimated values from the PSRREs presented in figures 9 and 10, and data for both hydrologic regions document a fairly uniform distribution around the line of equality. The greatest reduction in SEP for the PSRREs was observed when additional basin characteristics were included in the PSRREs, particularly land-use characteristics that had never been analyzed in previous studies such as amount of soil consisting of clay in the basin in percent. Dividing eastern Colorado at $-104^{\circ}$ longitude into two separate hydrologic regions dramatically reduced the $S E P$ for the Foothills hydrologic region, whereas in the Plains hydrologic region a less pronounced reduction in the $S E P$ was computed (fig. 8; table 3). Expanding the study from 50 miles beyond the State line, as was done in previous studies (McCain and Jarrett, 1976; Kircher and others, 1985; Vaill, 1999; Capesius and Stephens, 2009), to 100 miles was critical in significantly reducing the $S E P$ for the new Foothills hydrologic region from that computed for the 2009 Plains hydrologic region, and also slightly decreasing the $S E P$ in the new Plains hydrologic region.

Figure 12 plots the estimated $Q_{1 \%}$ AEPD from the PSRREs for the 188 streamgages used in the analysis from both the Foothills and Plains hydrologic region with the estimated $Q_{1 \%}$ AEPD from the PSRREs published in Capesius and Stephens (2009). In general, the PSRREs presented in the current (2015) report reduce the estimated $Q_{1 \%}$ AEPD at the 188 streamgages where both sets of PSRREs were tested, particularly when the $Q_{1 \%}$ AEPDs approach or exceed 20,000 cubic feet per second $\left(\mathrm{ft}^{3} / \mathrm{s}\right)$.

Table 3. Standard error of prediction of the peak-streamflow regional-regression equations for eastern Colorado presented in the previous study (Capesius and Stephens, 2009), Foothills hydrologic region, and Plains hydrologic region.

$\left[S E P\right.$, standard error of prediction; $Q_{0.5}, Q_{0.2}, Q_{0.1}, Q_{0.04}, Q_{0.02}, Q_{0.01}, Q_{0.005^{\prime}}, Q_{0.002^{\prime}}$ discharge with an annual exceedance-probability of $0.5,0.2,0.1,0.04,0.02$, $0.01,0.005,0.002$, respectively]

\begin{tabular}{cccc}
\hline $\begin{array}{c}\text { Annual } \\
\text { exceedance- } \\
\text { probability } \\
\text { discharge }\end{array}$ & $\begin{array}{c}\text { Previous SEP } \\
\text { (69 streamgages) }\end{array}$ & $\begin{array}{c}\text { Foothills SEP } \\
\text { (89 streamgages) }\end{array}$ & $\begin{array}{c}\text { Plains SEP } \\
\text { (99 streamgages) }\end{array}$ \\
\hline$Q_{0.5}$ & 183 & 117 & 131 \\
$Q_{0.2}$ & 142 & 87 & 102 \\
$Q_{0.1}$ & 136 & 80 & 103 \\
$Q_{0.04}$ & 137 & 80 & 113 \\
$Q_{0.02}$ & 139 & 83 & 123 \\
$Q_{0.01}$ & 141 & 88 & 136 \\
$Q_{0.005}$ & 160 & 94 & 150 \\
$Q_{0.002}$ & 141 & 104 & 170 \\
Mean & 147 & 92 & 129 \\
\hline
\end{tabular}




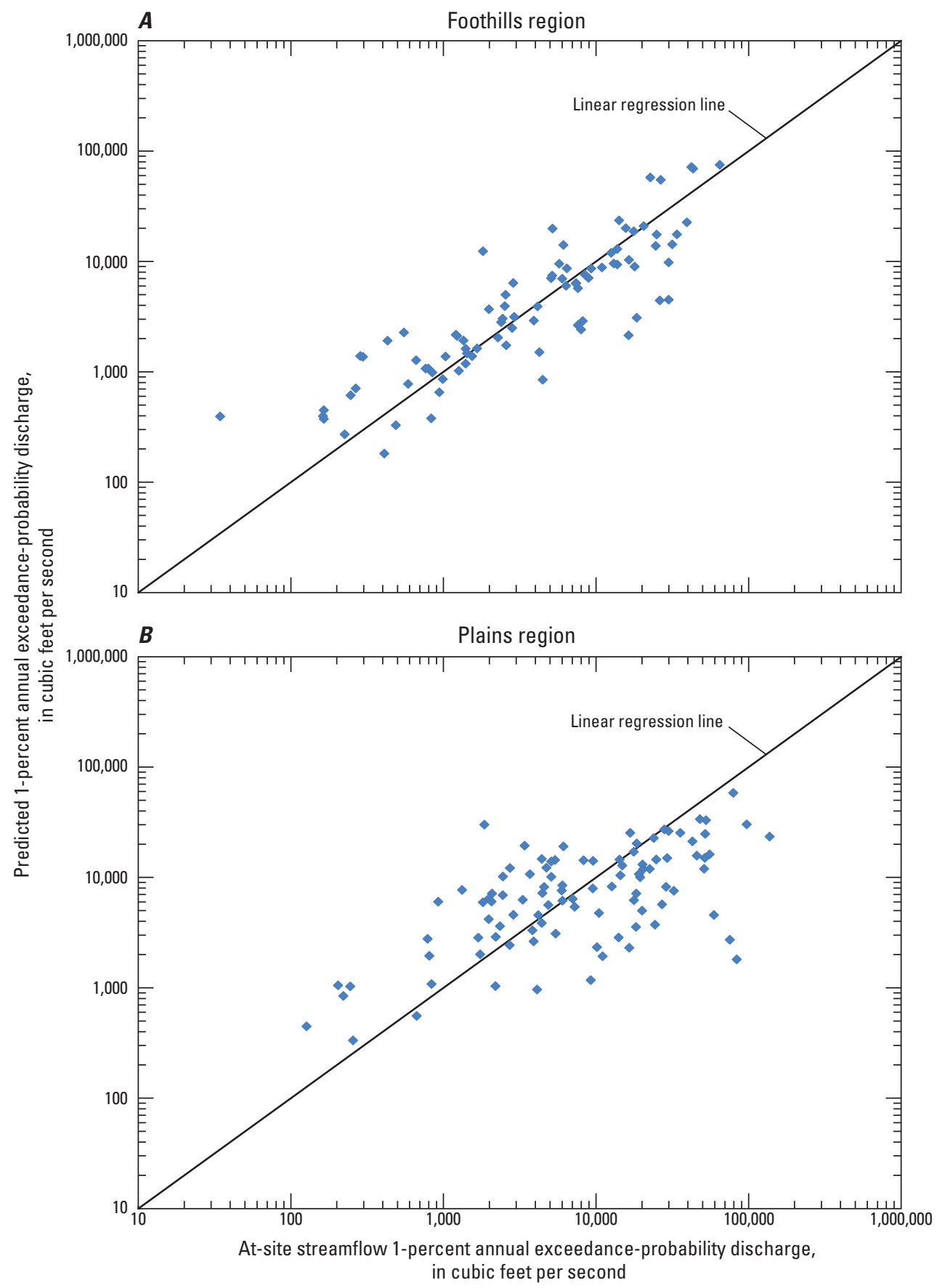

Figure 11. Relation between 1-percent annual exceedance-probability discharges computed from at-site streamflow to those predicted from the peak-streamflow regional-regression equations for the Foothills and Plains hydrologic regions of Colorado. 


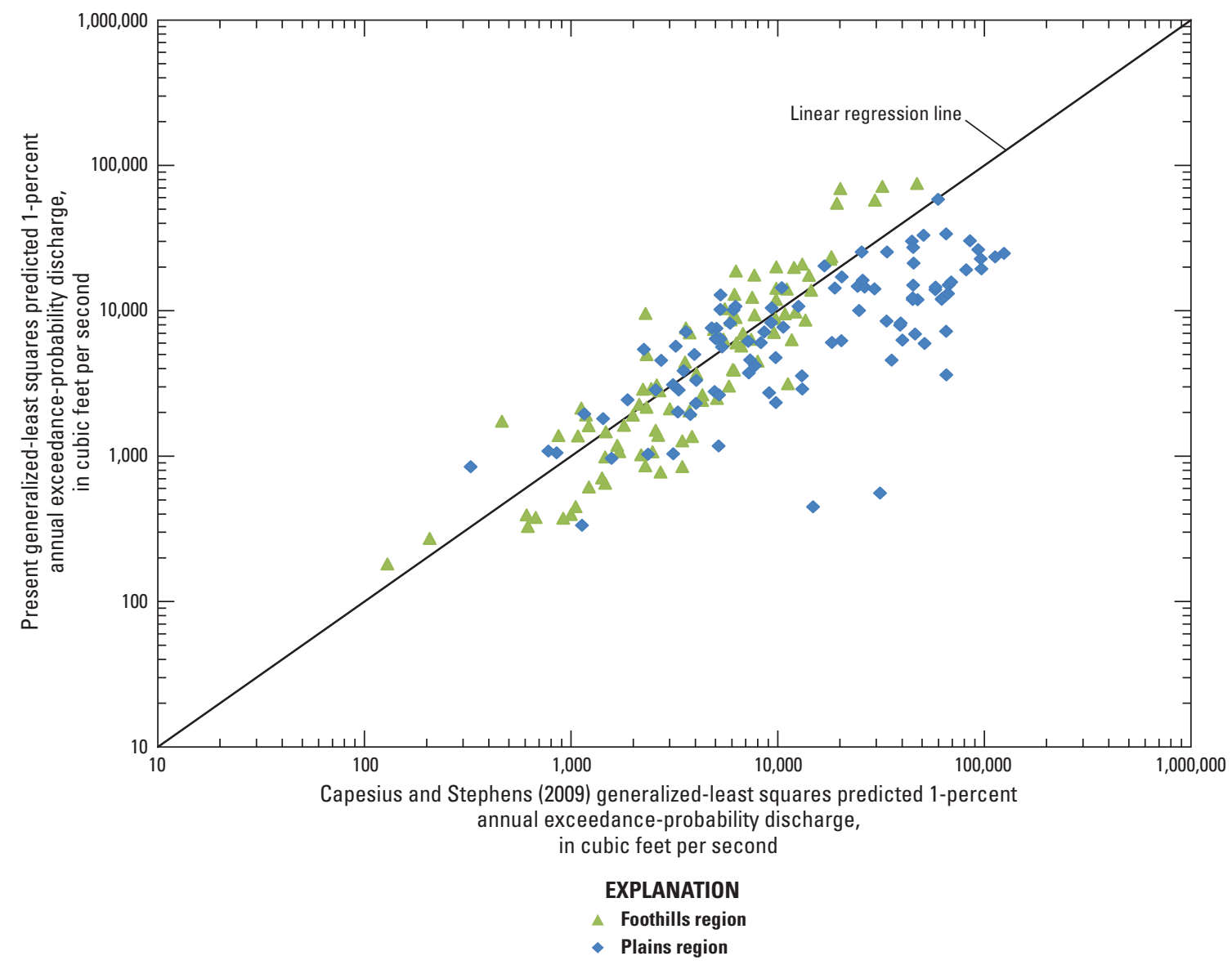

Figure 12. Relation between 1-percent annual exceedance-probability discharges computed from the peakstreamflow regional-regression equations in the Foothills and Plains hydrologic regions of Colorado and the 1-percent annual exceedance-probability discharges computed from the peak-streamflow regional-regression equations in Capesius and Stephens (2009).

\section{Application and Limitations of Peak-Streamflow Regional-Regression Equations}

This section provides four methods to estimate AEPDs at streams in eastern Colorado. The best method may depend on several factors: (1) if the site of interest is located at a streamgage and the record length and quality at a streamgage, where weighting of different estimates can result in more reliable AEPDs, (2) the drainage-area ratio between the site of interest and the streamgage, if the site of interest is on the same stream as a streamgage, (3) if the streamgage data are representative of the flow characteristics at the site of interest, and (4) whether or not the site of interest spans more than one hydrologic region.

\section{Use of Peak-Streamflow Regional-Regression Equations at Streamgages}

Improved estimates of AEPDs at streamgages can be obtained by weighting the AEPD EMA/MGB estimate with the PSRRE estimate as recommended by Bulletin 17B
(Interagency Advisory Committee on Water Data, 1982). The variance of prediction is a measure of the uncertainty of both estimates and can be used to lower the uncertainty of the weighted estimate by weighting the variance of prediction of both estimates, which are inversely proportional to their associated estimates of peak flow (Gotvald and others, 2012). Assuming the estimates from PeakFQ and the PSRREs are independent, the variance of the weighted estimate will be less than the variance of either of the independent estimates (Gotvald and others, 2012). Optimal weighted estimates of AEPDs were computed for this study in Appendix 5 using the AEPDs and the corresponding variances determined by PeakFQ and WREG.

After both the variances have been computed, the two independent annual exceedance-probability estimates can be weighted using the following equation (Verdi and Dixon, 2011; Cohn and others, 2012; Gotvald and others, 2012).

$$
\log Q_{P(g) w}=\frac{V P_{P(g) r} \log Q_{P(g) s}+V P_{P(g) s} \log Q_{P(g) r}}{V P_{P(g) s}+V P_{P(g) r}}
$$


where

$$
\begin{aligned}
& Q_{P(g) w} \quad \text { is the weighted independent estimate of } \\
& \text { annual peak flow for the selected } P \text {-percent } \\
& \text { AEP for a streamgage, } g \text {, in cubic feet per } \\
& \text { second (Appendix 5); } \\
& V P_{P(g) r} \quad \text { is the variance of prediction at the streamgage }
\end{aligned}
$$

Weighting the variances inversely proportional minimizes the effect of an estimate with high uncertainty; likewise, if the uncertainty is low then the weight of the estimate is large (Southard and Veilleux, 2014). The computed variance of prediction associated with the weighted estimate, $V P_{P(g) w}$, is shown in the following equation (Verdi and Dixon, 2011; Gotvald and others, 2012):

$$
V P_{P(g) w}=\frac{V P_{P(g) s} V P_{P(g) r}}{V P_{P(g) s}+V P_{P(g) r}}
$$

where the variables are previously defined. The weighted AEPDs estimates that were computed from equation 6 , the variance of prediction values derived from the applicable PSRREs, the PeakFQ AEPDs, the variance of prediction values for the 188 streamgages included in this study, and the peak flow estimate from the PSRREs are listed in Appendix 5.

\section{Use of Peak-Streamflow Regional-Regression Equations on Gaged Streams}

Sites of interest on streams with streamgages may have estimates determined by area weighting the AEPDs on the basis of the drainage-area ratio between the site of interest and the streamgage on the same stream (Olson, 2014). The weighting procedure is not applicable when the drainage-area ratio is less than 0.5 or greater than 1.5 , or when the flood characteristics substantially change between sites (Eash and others, 2013). To compute the area-weighted estimate at the ungaged site, the $Q_{P(g) w}$ estimate (Appendix 5) for the streamgage from equation 6 must be computed, then the area-weighted AEPD for the ungaged site, $Q_{P(u) a w}$, is then computed using the following equation (Olson, 2014):

$$
Q_{P(u) a w}=\left(\frac{A_{(u)}}{A_{(g)}}\right)^{b} Q_{P(g) w}
$$

\begin{tabular}{|c|c|c|c|c|}
\hline \multirow{2}{*}{$\begin{array}{c}\text { Annual } \\
\text { exceedance } \\
\text { probability }\end{array}$} & \multicolumn{2}{|c|}{$\begin{array}{c}\text { Foothills } \\
\text { hydrologic region }\end{array}$} & \multicolumn{2}{|c|}{$\begin{array}{c}\text { Plains } \\
\text { hydrologic region }\end{array}$} \\
\hline & Exponent $\boldsymbol{b}$ & Constant & Exponent $b$ & Constant \\
\hline 0.5 & 0.674 & 1.237 & 0.333 & 1.793 \\
\hline 0.2 & 0.642 & 1.720 & 0.331 & 2.305 \\
\hline 0.1 & 0.628 & 1.969 & 0.333 & 2.556 \\
\hline 0.04 & 0.616 & 2.231 & 0.337 & 2.817 \\
\hline 0.02 & 0.609 & 2.395 & 0.340 & 2.985 \\
\hline 0.01 & 0.602 & 2.543 & 0.344 & 3.134 \\
\hline 0.005 & 0.597 & 2.673 & 0.348 & 3.268 \\
\hline 0.002 & 0.592 & 2.828 & 0.354 & 3.428 \\
\hline
\end{tabular}

where

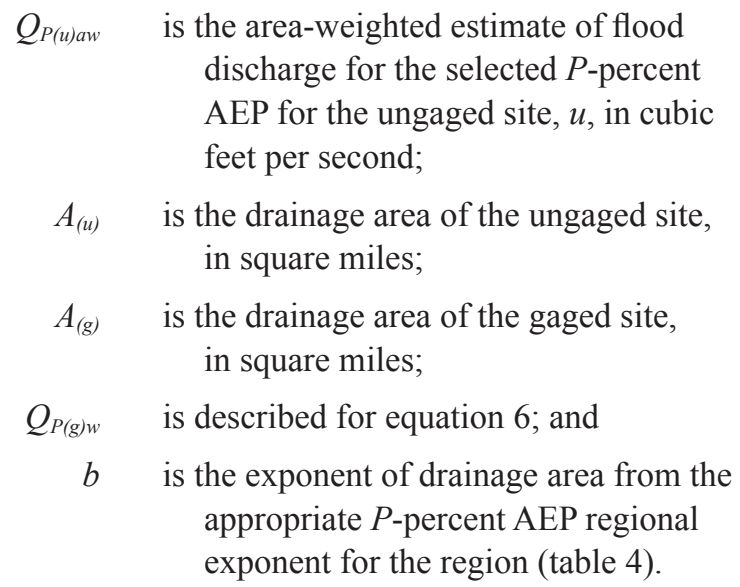

Following Southard and Veilleux (2014), a GLS analysis using only drainage area $(A)$ as an independent variable was performed to define the regional exponent for area-weighted estimates, and the resulting regional exponents ranged from 0.592 to 0.674 for the Foothills hydrologic region and from 0.331 to 0.354 for Plains hydrologic region. The resulting regional constants ranged from 1.237 to 2.828 for the Foothills hydrologic region and from 1.793 to 3.428 for Plains hydrologic region (table 4). The regional constants represent the unit drainage area for a given flood-frequency event for the reference basin (Vogel and Sankarasubramanian, 2000; Farmer and others, 2015).

Table 4. Regional exponents and constants determined from regional regression of log-transformed drainage area for areaweighting method to estimate annual exceedance-probability discharges for ungaged sites on gaged streams.

$[b$, exponent of drainage area from the appropriate $P$-percent annual exceedanceprobability regional exponent for the region] 


\section{Use of Peak-Streamflow Regional-Regression Equations on Ungaged Streams}

The PSRREs can be used if the ungaged site meets the criteria for use of this method. The PSRREs presented in figures 9 and 10 are applicable for streams that are minimally affected by anthropogenic activities and urbanization within the basin. The applicable range of basin characteristics that are used as explanatory variables for the PSRREs is listed in figures 9 and 10. These PSRREs are to be used with caution at ungaged locations for which the basin characteristics are outside the range of those used to develop the PSRREs. These PSRREs also are to be used with caution at ungaged locations outside Colorado or outside the Foothills and Plains hydrologic region of Colorado. The Foothills hydrologic region has four basin-characteristic ranges for applying the PSRREs. For the Foothills hydrologic region, the applicable range for drainage area is from 0.60 to $2,850 \mathrm{mi}^{2}$, the applicable range for 6-hour, 100-year precipitation is from 2.38 to 4.89 in., the applicable range for amount of soil consisting of clay in basin is from 9.87 to 37.5 percent, and the applicable range for basin outlet elevation is from 4,290 to $8,270 \mathrm{ft}$. The Plains hydrologic region has three basincharacteristics ranges for applying the PSRREs. For the Plains hydrologic region, the applicable range for drainage area is from 0.26 to $3,560 \mathrm{mi}^{2}$, the applicable range for mean basin slope is from 0.41 to 21.9 percent, and the applicable range for amount of soil consisting of clay in basin is from 5.20 to 38.5 percent.

\section{Use of Peak-Streamflow Regional- Regression Equations on Ungaged Streams in Two Hydrologic Regions}

For an ungaged site on a stream that crosses hydrologic regions, the PSRREs for each region can be applied separately using basin characteristics for the entire drainage basin above the ungaged site (Gotvald and others, 2012). The individual peak flows from each region can then be weighted by the proportion of drainage area within each region and added to produce final estimates for the ungaged site. For example, if 25 percent of the drainage area at an ungaged site is in the upstream region and 75 percent is in the downstream region, the discharge estimate based on PSRREs for the upstream region are multiplied by 0.25 and added to 0.75 times the regression estimate based on PSRREs for the downstream region. The variance of prediction for such a weighted estimate can also be approximated by using the same weighting procedure based on proportional drainage areas (Gotvald and others, 2012).

\section{Effect of Paleoflood Data on the Peak- Streamflow Regional-Regression Equations}

The amount of information collected from paleoflood data varied across the 44 streamgages where paleoflood data were collected. For some sites, the paleoflood data were informative and added hundreds or even thousands of years of record, where at other sites the information collected was minimal with only a decade or two added to the period of record. Appendix 2 lists the estimated discharge, uncertainty of the discharge, age, and uncertainty of the age for all the paleoflood data used in the analysis at the 44 streamgages where paleoflood data were available. Paleoflood data can be used in the context of the EMA to extend the record length and the amount of flood information known at a streamgage. While not comparable to adding equivalent years of systematic record, the addition of up to hundreds of years of paleoflood data to a streamgage can substantially improve the low probability AEPDs. Increasing the record length at streamgages where paleoflood data are collected provides more weight to these streamgages when the GLS regression is performed. The new record length created by the paleoflood data, the effective record length, is determined from the age of the paleoflood data and the AEPD and also includes the existing systematic data; the effective record length was computed using computer programming code written in R (Venables and Smith, 2014) following techniques outlined in Stedinger and Cohn (1986) and Cohn and others (2001). Table 5 presents the record length and resulting effective record length added for each AEPD at the 44 streamgages where paleoflood data were available. Table 6 presents the record length by hydrologic region and the record length added by including paleoflood data at 44 of the 188 streamgages. While the mean age of paleoflood data at the 44 streamgages was 630 years (Appendix 2), that only resulted in a mean of 28 and 21 years (table 6) of increase in effective record length for the 1-percent AEPD when included with all 89 and 99 streamgages in the Foothills and Plains hydrologic regions, respectively. In addition, 24 of the 44 streamgages with paleoflood data have effective record lengths of 100 years or less even after the addition of the paleoflood data for the 1-percent AEPD (table 5).

At the 44 streamgages where paleoflood data were available, the mean record length prior to the inclusion of paleoflood data was 23 and 30 years in the Foothills and Plains hydrologic regions, respectively (tables 5 and 6). After the inclusion of the paleoflood data, the mean record length increased to 24-363 years (depending on AEPD) and 30-268 years (depending on AEPD) in the Foothills and Plains hydrologic regions, respectively (table 5). With the increase in record length, the mean SEP of the 8 AEPDs was reduced from 145 to 129 percent (10.7 percent mean reduction) and even more substantially for AEPDs of 1-percent and less (100-year flood discharge and greater) when the paleoflood data were added to the analyses 
Table 5. Number of years of record at the 44 streamgages where paleoflood data were collected.

[USGS, U.S. Geological Survey; $Q_{0.5}, Q_{0.2}, Q_{0.1}, Q_{0.04}, Q_{0.02}, Q_{0.01}, Q_{0.005}, Q_{0.002}$, discharge with an annual exceedance-probability of $0.5,0.2,0.1,0.04,0.02,0.01,0.005,0.002$, respectively; NA, not applicable]

\begin{tabular}{|c|c|c|c|c|c|c|c|c|c|c|c|c|c|}
\hline $\begin{array}{c}\text { USGS } \\
\text { streamgage } \\
\text { number }\end{array}$ & Streamgage name & $\begin{array}{l}\text { Hydro- } \\
\text { logic } \\
\text { region }\end{array}$ & $\begin{array}{c}\text { Number of } \\
\text { systematic } \\
\text { peaks }\end{array}$ & $\begin{array}{c}\text { Number of } \\
\text { historic } \\
\text { peaks }\end{array}$ & $\begin{array}{c}\text { Number of } \\
\text { paleoflood } \\
\text { peaks }\end{array}$ & $\begin{array}{c}\boldsymbol{Q}_{0.5} \\
\text { effective } \\
\text { record } \\
\text { length }\end{array}$ & $\begin{array}{c}\boldsymbol{Q}_{0.2} \\
\text { effective } \\
\text { record } \\
\text { length }\end{array}$ & $\begin{array}{c}\boldsymbol{Q}_{0.1} \\
\text { effective } \\
\text { record } \\
\text { length }\end{array}$ & $\begin{array}{c}\boldsymbol{Q}_{0.04} \\
\text { effective } \\
\text { record } \\
\text { length }\end{array}$ & $\begin{array}{c}\boldsymbol{Q}_{0.02} \\
\text { effective } \\
\text { record } \\
\text { length }\end{array}$ & $\begin{array}{c}\boldsymbol{Q}_{0.01} \\
\text { effective } \\
\text { record } \\
\text { length }\end{array}$ & $\begin{array}{c}\boldsymbol{Q}_{0.005} \\
\text { effective } \\
\text { record } \\
\text { length }\end{array}$ & $\begin{array}{c}\boldsymbol{Q}_{0.002} \\
\text { effective } \\
\text { record } \\
\text { length }\end{array}$ \\
\hline 06687000 & Blue Creek near Lewellen, Nebr. & Plains & 81 & 0 & 1 & 80 & 81 & 81 & 81 & 81 & 81 & 80 & 80 \\
\hline 06712000 & Cherry Creek near Franktown, Colo. & Foothills & 74 & 0 & 1 & 77 & 79 & 105 & 194 & 328 & 548 & 888 & 1,574 \\
\hline 06712500 & Cherry Creek near Melvin, Colo. & Foothills & 30 & 0 & 1 & 31 & 32 & 43 & 89 & 169 & 334 & 692 & 2043 \\
\hline 06719500 & Clear Creek near Golden, Colo. & Foothills & 62 & 0 & 1 & 64 & 64 & 65 & 67 & 68 & 69 & 69 & 69 \\
\hline 06755960 & Crow Creek at 19th Street, at Cheyenne, Wyo. & Foothills & 23 & 0 & 1 & 22 & 30 & 45 & 73 & 89 & 96 & 96 & 93 \\
\hline 06758200 & Kiowa Creek at Kiowa, Colo. & Foothills & 10 & 0 & 1 & 11 & 15 & 23 & 45 & 64 & 79 & 83 & 82 \\
\hline 06758700 & Middle Bijou Creek Tributary near Deer Trail, Colo. & Foothills & 10 & 0 & 1 & 12 & 18 & 31 & 82 & 156 & 234 & 266 & 247 \\
\hline 06762600 & Lodgepole Creek Tributary Number 2 near Albin, Wyo. & Foothills & 25 & 0 & 1 & 25 & 25 & 27 & 30 & 32 & 33 & 34 & 35 \\
\hline 06763500 & Lodgepole Creek at Ralton, Nebr. & Plains & 36 & 1 & 1 & 37 & 41 & 51 & 65 & 72 & 76 & 77 & 78 \\
\hline 06831500 & Frenchman Creek near Imperial, Nebr. & Plains & 31 & 0 & 1 & 31 & 36 & 45 & 59 & 66 & 69 & 70 & 70 \\
\hline 06835100 & Bobtail Creek near Palisade, Nebr. & Plains & 13 & 0 & 1 & 13 & 17 & 26 & 54 & 92 & 141 & 189 & 231 \\
\hline 06844800 & South Fork Sappa Creek Tributary near Goodland, Kans. & Plains & 33 & 0 & 1 & 34 & 39 & 46 & 55 & 59 & 61 & 61 & 61 \\
\hline 06858500 & North Fork Smoky Hill River near McAllaster, Kans. & Plains & 33 & 0 & 1 & 33 & 36 & 48 & 85 & 130 & 180 & 225 & 264 \\
\hline 07099215 & Turkey Creek near Fountain, Colo. & Foothills & 30 & 0 & 1 & 30 & 34 & 44 & 72 & 97 & 119 & 134 & 144 \\
\hline 07099220 & Little Turkey Creek near Fountain, Colo. & Foothills & 10 & 0 & 1 & 10 & 11 & 14 & 22 & 31 & 41 & 50 & 60 \\
\hline 07103703 & Camp Creek at Garden of the Gods, Colo. & Foothills & 22 & 0 & 1 & 23 & 29 & 42 & 72 & 97 & 115 & 125 & 128 \\
\hline 07103960 & Kettle Creek above U.S. Air Force Academy, Colo. & Foothills & 13 & 0 & 2 & 13 & 14 & 18 & 27 & 35 & 44 & 50 & 57 \\
\hline 07103977 & Cottonwood Creek at Cowpoke Road at Colorado Springs, Colo. & Foothills & 11 & 0 & 1 & 11 & 11 & 11 & 11 & 11 & 10 & 10 & 10 \\
\hline 07105000 & Bear Creek near Colorado Springs, Colo. & Foothills & 22 & 0 & 2 & 22 & 22 & 24 & 28 & 31 & 33 & 35 & 37 \\
\hline 07105490 & Cheyenne Creek at Evans Avenue at Colorado Springs, Colo. & Foothills & 22 & 0 & 1 & 22 & 24 & 28 & 35 & 40 & 44 & 47 & 48 \\
\hline 07105940 & Little Fountain Creek near Fountain, Colo. & Foothills & 12 & 0 & 1 & 13 & 17 & 24 & 39 & 52 & 64 & 71 & 77 \\
\hline 07107500 & St. Charles River at Burnt Mill, Colo. & Foothills & 27 & 0 & 2 & 28 & 35 & 49 & 78 & 100 & 116 & 124 & 126 \\
\hline 07120620 & Big Arroyo near Thatcher, Colo. & Foothills & 30 & 0 & 1 & 31 & 33 & 47 & 103 & 210 & 447 & 972 & 2,596 \\
\hline 07124300 & Long Canyon Creek near Madrid, Colo. & Foothills & 18 & 0 & 1 & 18 & 19 & 22 & 30 & 35 & 38 & 40 & 41 \\
\hline 07125100 & Frijole Creek near Alfalfa, Colo. & Foothills & 13 & 2 & 2 & 21 & 32 & 47 & 66 & 71 & 71 & 69 & 65 \\
\hline 07125500 & San Francisco Creek near Alfalfa, Colo. & Foothills & 15 & 0 & 2 & 16 & 19 & 28 & 54 & 83 & 111 & 131 & 142 \\
\hline 07126200 & Van Bremer Arroyo near Model, Colo. & Plains & 48 & 0 & 1 & 48 & 50 & 58 & 74 & 85 & 92 & 97 & 100 \\
\hline 07126325 & Taylor Arroyo below Rock Crossing, near Thatcher, Colo. & Plains & 31 & 0 & 1 & 31 & 35 & 45 & 69 & 89 & 106 & 118 & 125 \\
\hline 07126390 & Lockwood Canyon Creek near Thatcher, Colo. & Plains & 30 & 0 & 1 & 32 & 40 & 52 & 71 & 80 & 83 & 83 & 82 \\
\hline 07126415 & Red Rock Canyon Creek at Mouth near Thatcher, Colo. & Plains & 31 & 0 & 1 & 31 & 31 & 33 & 35 & 37 & 38 & 38 & 39 \\
\hline 07126480 & Bent Canyon Creek at Mouth near Timpas, Colo. & Plains & 29 & 0 & 1 & 29 & 32 & 42 & 64 & 83 & 99 & 110 & 118 \\
\hline 07134100 & Big Sandy Creek near Lamar, Colo. & Plains & 33 & 1 & 1 & 34 & 39 & 46 & 55 & 60 & 62 & 64 & 64 \\
\hline 07135000 & Two Butte Creek near Holly, Colo. & Plains & 10 & 0 & 1 & 12 & 17 & 29 & 70 & 126 & 181 & 204 & 195 \\
\hline 07138600 & White Woman Creek Tributary near Selkirk, Kans. & Plains & 39 & 0 & 1 & 39 & 43 & 54 & 82 & 107 & 130 & 148 & 164 \\
\hline 07138650 & White Woman Creek near Leoti, Kans. & Plains & 20 & 0 & 1 & 20 & 25 & 37 & 83 & 164 & 321 & 587 & 1,016 \\
\hline 07155100 & Cold Springs Creek near Wheeless, Okla. & Plains & 17 & 1 & 1 & 19 & 24 & 35 & 60 & 81 & 97 & 105 & 106 \\
\hline 07156100 & Sand Arroyo Creek near Johnson, Kans. & Plains & 15 & 0 & 1 & 16 & 20 & 31 & 72 & 145 & 274 & 447 & 601 \\
\hline 07156220 & Bear Creek near Johnson, Kans. & Plains & 32 & 0 & 1 & 32 & 37 & 47 & 64 & 75 & 82 & 85 & 86 \\
\hline 07156600 & Cimarron River Tributary near Moscow, Kans. & Plains & 33 & 0 & 2 & 34 & 36 & 44 & 63 & 77 & 87 & 94 & 98 \\
\hline 07201200 & Chicorica Creek Tributary near Raton, N. Mex. & Foothills & 31 & 0 & 1 & 31 & 34 & 44 & 71 & 98 & 126 & 149 & 171 \\
\hline 07201450 & Green Mountain Arroyo near Raton, N. Mex. & Foothills & 12 & 0 & 1 & 13 & 19 & 32 & 72 & 122 & 172 & 199 & 198 \\
\hline 07203600 & Rio Del Plano Tributary near Taylor Springs, N. Mex. & Foothills & 12 & 1 & 2 & 13 & 15 & 20 & 40 & 69 & 118 & 191 & 317 \\
\hline 07227300 & Sand Draw near Clayton, N. Mex. & Plains & 18 & 0 & 1 & 19 & 22 & 32 & 70 & 138 & 281 & 565 & 1,253 \\
\hline 07232650 & Aqua Frio Creek near Felt, Okla. & Plains & 12 & 0 & 2 & 13 & 16 & 23 & 50 & 95 & 185 & 356 & 799 \\
\hline Foothills mean & NA & NA & 23 & 0 & 1 & 24 & 27 & 36 & 61 & 91 & 133 & 197 & 363 \\
\hline Plains mean & NA & NA & 30 & 0 & 1 & 30 & 34 & 43 & 66 & 92 & 130 & 181 & 268 \\
\hline Mean & NA & NA & 26 & 0 & 1 & 27 & 31 & 40 & 63 & 92 & 132 & 189 & 318 \\
\hline
\end{tabular}


Table 6. Record length of the 188 streamgages by region used in the analysis by hydrologic region and the effect of paleoflood data on the record length for various annual exceedance-probability discharges.

$\left[Q_{0.5}, Q_{0.2}, Q_{0.1}, Q_{0.04}, Q_{0.02}, Q_{0.01}, Q_{0.005}, Q_{0.002}\right.$, discharge with an annual exceedance-probability of $0.5,0.2,0.1,0.04,0.02,0.01,0.005,0.002$, respectively]

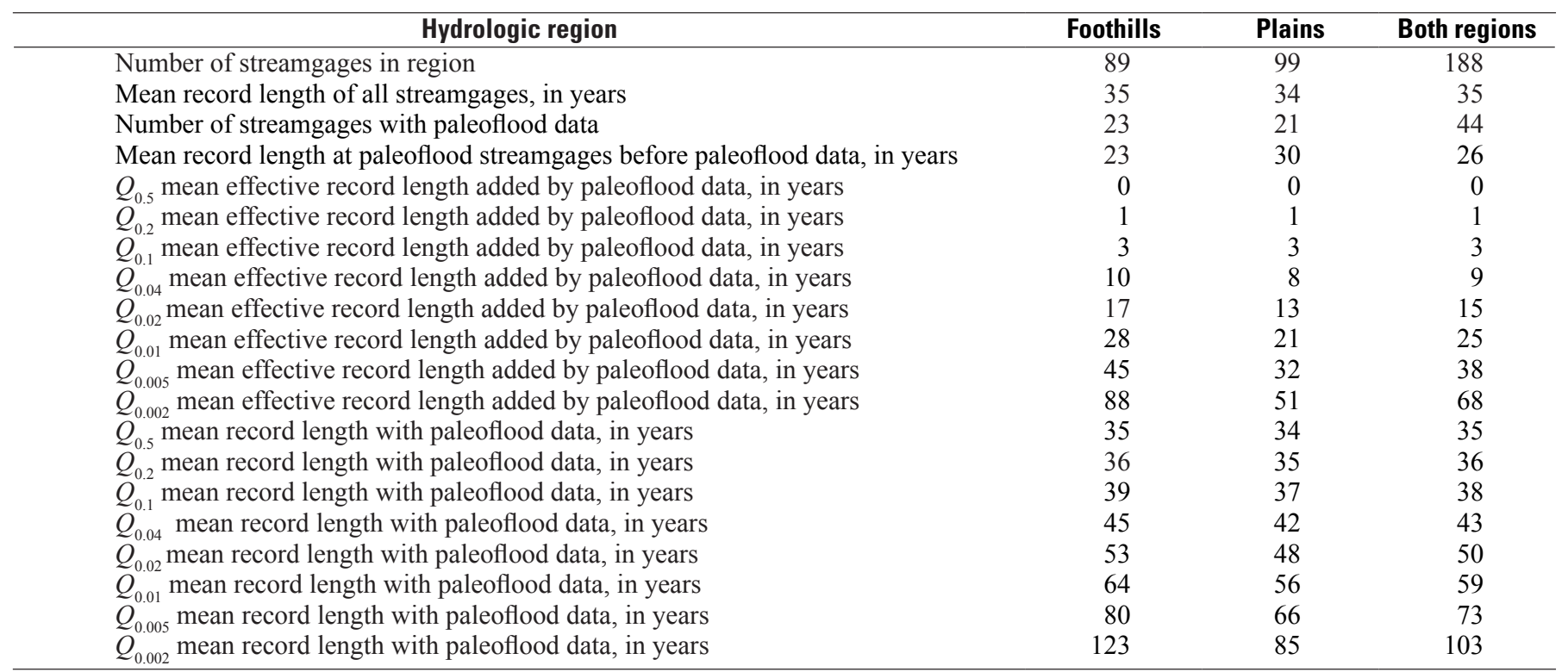

Table 7. Standard error of prediction of the peak-streamflow regional-regression equations for eastern Colorado across both hydrologic regions for the 44 streamgages where paleoflood data were collected with and without the inclusion of the paleoflood data.

$\left[S E P\right.$, standard error of prediction; $Q_{0.5}, Q_{0.2}, Q_{0.1}, Q_{0.04}, Q_{0.02}, Q_{0.01}, Q_{0.005}, Q_{0.002}$, discharge with an annual exceedance probability of $0.5,0.2$, $0.1,0.04,0.02,0.01,0.005,0.002$, respectively; \%, percent]

\begin{tabular}{cccc}
\hline $\begin{array}{c}\text { Annual } \\
\text { exceedance- } \\
\text { probability discharge }\end{array}$ & $\begin{array}{c}\text { SEP without paleoflood data } \\
\text { across both hydrologic regions } \\
\text { (44 streamgages) }\end{array}$ & $\begin{array}{c}\text { SEP with paleoflood data } \\
\text { across both hydrologic regions } \\
\text { (44 streamgages) }\end{array}$ & $\begin{array}{c}\text { Mean change from } \\
\text { previous SEP } \\
\text { (percent) }\end{array}$ \\
\hline$Q_{0.5}$ & 166 & 157 & -5.5 \\
$Q_{0.2}$ & 132 & 123 & -6.3 \\
$Q_{0.1}$ & 126 & 117 & -7.6 \\
$Q_{0.04}$ & 129 & 117 & -9.1 \\
$Q_{0.02}$ & 135 & 121 & -10.5 \\
$Q_{0.01}$ & 143 & 126 & -12.3 \\
$Q_{0.005}$ & 154 & 132 & -14.4 \\
$Q_{0.002}$ & 172 & 141 & -18.0 \\
Mean & 145 & 129 & -10.7 \\
\hline
\end{tabular}

(table 7). When all 188 streamgages were considered, including the paleoflood data at the 44 streamgages, the record length was increased from 35 and 34 years to 35-123 years (depending on AEPD) and 34-85 years (depending on AEPD) in the Foothills and Plains hydrologic regions, respectively (table 6). However, even with a substantial increase in mean record length from paleoflood data, the paleoflood data had minimal effect on the SEP of the AEPDs (table 8). With the addition of the paleoflood data at 44 of the sites, the mean $S E P$ for the Foothills hydrologic region went from 95 to 92 percent, the mean SEP for the Plains hydrologic region went from 128 to 129 percent, and the mean SEP for both hydrologic regions went from 137 to 139 percent (table 8). These results lead the authors to conclude that for paleoflood data to be effective in reducing the SEP in the
Foothills and Plains hydrologic regions of eastern Colorado, a larger ratio than 44 of 188 ( 23 percent) streamgages would need paleoflood data and that paleoflood data would need to increase the record length by more than 25 years for the 1-percent AEPD. However, in other regions where the hydrology is less variable, it may be possible to collect paleoflood data at a similar fraction of the streamgages in a hydrologic region with a similar increase in record length and obtain significant reduction in the uncertainty in the PSRREs. To make further reductions in the uncertainties of the PSRREs in the Foothills and Plains hydrologic regions, additional streamgages or crest-stage gages are needed to collect peak-streamflow data on natural streams in eastern Colorado. 
Table 8. Standard error of prediction of the peak-streamflow regional-regression equations for eastern Colorado with and without the inclusion of the paleoflood data.

$\left[S E P\right.$, standard error of prediction; $Q_{0.5}, Q_{0.2}, Q_{0.1}, Q_{0.04}, Q_{0.02}, Q_{0.01}, Q_{0.005}, Q_{0.002}$, discharge with an annual exceedance probability of $0.5,0.2,0.1,0.04,0.02,0.01$, $0.005,0.002$, respectively]

\begin{tabular}{|c|c|c|c|c|c|c|}
\hline $\begin{array}{c}\text { Annual } \\
\text { exceedance- } \\
\text { probability } \\
\text { discharge } \\
\text { (percent) }\end{array}$ & $\begin{array}{l}\text { SEP without paleo- } \\
\text { flood data for both } \\
\text { hydrologic regions } \\
\text { (188 streamgages) }\end{array}$ & $\begin{array}{l}\text { SEP with paleo- } \\
\text { flood data for both } \\
\text { hydrologic regions } \\
\text { (188 streamgages) }\end{array}$ & $\begin{array}{c}\text { Foothills } S E P \\
\text { without } \\
\text { paleoflood data } \\
\text { (89 streamgages) }\end{array}$ & $\begin{array}{l}\text { Foothills SEP with } \\
\text { paleoflood data } \\
\text { (89 streamgages) }\end{array}$ & $\begin{array}{c}\text { Plains SEP } \\
\text { without } \\
\text { paleoflood data } \\
\text { (99 streamgages) }\end{array}$ & $\begin{array}{l}\text { Plains SEP with } \\
\text { paleoflood data } \\
\text { (99 streamgages) }\end{array}$ \\
\hline$Q_{05}$ & 154 & 156 & 117 & 117 & 128 & 131 \\
\hline$Q_{0.5}$ & 121 & 124 & 87 & 87 & 99 & 102 \\
\hline$Q_{0.1}^{0.2}$ & 117 & 121 & 81 & 80 & 101 & 103 \\
\hline$Q_{0.01}^{0.02}$ & 138 & 140 & 94 & 88 & 137 & 136 \\
\hline$Q_{0.005}^{0.01}$ & 149 & 150 & 101 & 94 & 153 & 150 \\
\hline$Q_{0.002}$ & 166 & 164 & 114 & 104 & 175 & 170 \\
\hline Mean & 137 & 139 & 95 & 92 & 128 & 129 \\
\hline
\end{tabular}

\section{StreamStats}

The USGS has developed a Web-based computer program, StreamStats, (http://water.usgs.gov/osw/streamstats/ index.html) (Ries and others, 2004; USGS, 2015) which facilitates the computation of various streamflow statistics, including peak streamflow, using regional-regression equations published in this and previous reports (Capesius and Stephens, 2009). StreamStats allows the user to obtain streamflow statistics for both gaged and ungaged sites by selecting a specific stream location on a map interface. If a user selects the location of a USGS streamgage, the user will receive previously published information for the streamgage from a database. If the location of interest lacks a streamgage, StreamStats delineates the basin upstream from the selected location, computes basin and climatic characteristics, and provides estimates of the streamflow statistics using the regionalregression equations. The results are presented in a table and a map showing the basin-boundary outline. The estimates are applicable for stream sites not significantly affected by regulation, diversions, channelization, backwater, or urbanization. In the past, it could take an experienced person more than a day to estimate this information at an ungaged site. StreamStats reduces the effort to only a few minutes.

StreamStats makes the process of computing streamflow statistics for ungaged sites much faster, more accurate, and more consistent than previously used manual methods (Eash and others, 2013). It also makes streamflow statistics for streamgages available without the need to locate, obtain, and read the publications in which streamflow statistics were originally provided. Examples of streamflow statistics that can be provided by StreamStats include the $Q_{1 \%}$ flood discharge, the mean-daily and mean-annual streamflow, and the mean 7-day, 10-year low streamflow. Examples of basin characteristics include the drainage area, basin elevation, mean-annual precipitation, percent of area underlain by hydrologic soil types, and so forth. Basin characteristics provided by StreamStats are the physical, geologic, and climatic properties that have been statistically related to movement of water through a drainage basin to a stream site.

Explanatory variables used to develop the PSRREs presented in this report are compatible for use with StreamStats. Finally, PSRREs developed in this report for Colorado have been included in StreamStats to ease the computation of estimates of peak streamflow for users.

\section{Summary}

The U.S. Geological Survey (USGS), in cooperation with the Colorado Department of Transportation, developed regional-regression equations for estimating the 50-, 20-, 10-, 4-, 2-, 1-, 0.5-, 0.2-percent annual exceedance-probability discharge (AEPD) for natural streamflow in eastern Colorado. At the completion of the selection process, 37 streamgages were excluded from the analysis because of redundancy, and a total of 188 streamgages, consisting of 6,536 years of record and a mean of approximately 35 years of record per streamgage, were used to develop the peak-streamflow regional-regression equations. Paleoflood and non-exceedance bound ages were established based on reconnaissance-level methods. Multiple lines of evidence were used at each streamgage to arrive at a conclusion (age estimate) to add a higher degree of certainty to reconnaissance-level estimates. Paleoflood or non-exceedance bound evidence was documented at 41 streamgages, and 3 streamgages had previously collected paleoflood data. To determine the peak discharge of a paleoflood or non-exceedance bound, two different hydraulic models were used. The estimated AEPDs for each streamgage were computed using the USGS computer program PeakFQ. The AEPDs were determined using systematic data through water year 2013 (October 1, 2012, through 
September 30, 2013). Based on previous studies conducted in Colorado and neighboring States and on the availability of data, a total of 72 characteristics ( 57 basin and 15 climatic characteristics) were evaluated as candidate explanatory variables in the regression analysis.

After analyzing the study area for potential regional subdivisions, the new hydrologic region located between $-104^{\circ}$ longitude and the Kansas-Nebraska State line will still be designated the Plains hydrologic region in this report. The hydrologic region comprising the rest of eastern Colorado will be designated the Foothills hydrologic region. The western edge of the Foothills hydrologic region was established by Vaill in 1999, and this report follows that boundary. The western edge of the Foothills hydrologic region follows the 7,500-foot contour south from the Wyoming State line to the Chaffee-Fremont County line where the hydrologic region boundary follows the Chaffee-Fremont County line south across the Arkansas River and transitions up to the 9,000 -foot contour which is followed south to the New Mexico State line.

Generalized-Least Squares regression was used exclusively to compute the final peak-streamflow regionalregression equations for peak streamflow. Dividing eastern Colorado into two new individual regions at $-104^{\circ}$ longitude resulted in peak-streamflow regional-regression equations with the smallest standard error of prediction $(S E P)$.

The mean $S E P$ for all 8 AEPDs in both the Foothills and Plains hydrologic region was reduced approximately 25 percent compared to the previous study, from 147 percent to 92 percent in the Foothills hydrologic region and to 129 percent in the Plains hydrologic region. When all 188 streamgages were considered, including the paleoflood data at the 44 streamgages, the paleoflood data had minimal effect on the SEP of the AEPDs. With the addition of the paleoflood data at 44 of the sites, the mean $S E P$ for the Foothills hydrologic region went from 95 to 92 percent, the mean $S E P$ for the Plains hydrologic region went from 128 to 129 percent, and the mean SEP for both hydrologic regions went from 137 to 139 percent. For paleoflood data to be effective in eastern Colorado, a larger ratio than 44 of 188 (23 percent) streamgages would need paleoflood data and that paleoflood data would need to increase the record length by more than 25 years for the 1-percent AEPD. The greatest reduction in $S E P$ for the peak-streamflow regional-regression equations was observed when additional basin characteristics, particularly land-use characteristics that had never been analyzed in previous studies, were included in the peakstreamflow regional-regression equations and by dividing eastern Colorado into two separate regions. To make further reductions in the uncertainties of the PSRREs in the Foothills and Plains hydrologic regions, additional streamgages or creststage gages are needed to collect peak-streamflow data on natural streams in eastern Colorado.

\section{References Cited}

Allaby, Michael, 1998, A dictionary of plant sciences, accessed January 23, 2015, at http://www.encyclopedia.com/doc/ 107-chronosequence.html.

Anderson, K.C., Wells, S.G., and Graham, R.C., 2002, Pedogenesis of vesicular horizons, Cima volcanic field, Mojave Desert, California: Soil Science Society of America Journal, v. 66, p. $878-887$.

Asquith, W.H., Roussel, M.C., and Vrabel, Joseph, 2006, Statewide analysis of the drainage-area ratio method for 34 streamflow percentile ranges in Texas: U.S. Geological Survey Scientific Investigations Report 2006-5286, 34 p., 1 appendix.

Baker, V.R., 1987, Paleoflood hydrology and extreme flood events: Journal of Hydrology, v. 96, p. 79-99.

Baker, V.R., 1989, Magnitude and frequency of paleofloods, in Beven, K.J., and Carling, P., eds., Floods-Hydrological, sedimentological, and geomorphological implications: New York, John Wiley and Sons, p. 171-183.

Beschel, R.E., 1973, Lichens as a measure of the age of recent moraines: Arctic and Alpine Research, v. 5, no. 4, p. 303-309.

Birkeland, P.W., 1973, Use of relative age-dating methods in a stratigraphic study of rock glacier deposits, Mt Sopris, Colorado: Arctic and Alpine Research, v. 5, no. 4, p. 401-416.

Birkeland, P.W., 1999, Soils and geomorphology: New York, Oxford University Press, 448 p.

Blainey, J.B., Webb, R.H., Moss, M.E., and Baker, V.R., 2002, Bias and information content of paleoflood data in flood-frequency analysis, in House, P.K., Webb, R.H., Baker, V.R., and Levish, D.R., eds., Ancient floods, modern hazards - Principles and applications of paleoflood hydrology: Washington D.C., American Geophysical Union, Water Sciences and Application, v. 5, p.161-174.

Boner, F.C., and Stermitz, F., 1967, Floods of June 1964 in northwestern Montana: U.S. Geological Survey WaterSupply Paper 1840-B, 242 p.

Bonnin, G.M., Martin, D., Lin, B., Parzybok, T., Yekta, M., and Riley, D., 2011, Precipitation-frequency atlas of the United States: National Weather Service, National Oceanic and Atmospheric Administration Atlas 14, v. 1, version 5.0 Semiarid Southwest (Arizona, Southeast California, Nevada, New Mexico, Utah), accessed August 1, 2014, at KWW://www.nws.noaa.gov/oh/hdsc/PF_documents/ Atlas14_Volume1.pdf. 
Bradley, D.N., 2012, Slope-area computation program graphical user interface 1.0 - A preprocessing and postprocessing tool for estimating peak flood discharge using the slope-area method: U.S. Geological Survey Fact Sheet 2012-3112, 4 p.

Capesius, J.P., and Stephens, V.C., 2009, Regional regression equations for the estimation of natural streamflow statistics in Colorado: U.S. Geological Survey Scientific Investigations Report 2009-5136, 46 p.

Cohn, T.A., Lane, W.L., and Stedinger, J.R., 2001, Confidence intervals for Expected Moments Algorithm flood quantile estimates: Water Resources Research, v. 37, no. 6, p. 1695-1706.

Cohn, T.A., Berenbrock, Charles, Kiang, J.E., and Mason, R.R., Jr., 2012, Calculating weighted estimates of peak streamflow statistics: U.S. Geological Survey Fact Sheet 2012-2038, 4 p., at http://pubs.usgs.gov/fs/2012/3038/.

Cohn, T.A., England, J.F., Berenbrock, C.E., Mason, R.R., Stedinger, J.R., and Lamontagne, J.R., 2013, A generalized Grubbs-Beck test statistic for detecting multiple potentially influential low outliers in flood series: Water Resources Research, v. 49, no. 8, p. 5047-5058.

Colorado Division of Water Resources, 2013, Colorado's surface water conditions: Colorado Division of Water Resources Web site, accessed November 8, 2013, at http://www.dwr.state.co.us/SurfaceWater/Default.aspx.

Cook, R.U., 1970, Stone pavements in deserts: Annals of the Association of American Geographers, v. 60, p. 560-577.

Cornwell, K.J., 1986, Geomorphology and soils, in Adair, M.J., and Brown, K.L., eds., Prehistoric and historic cultural resources of selected sites at Harlan County Lake, Harlan County, Nebraska: Kansas City, Kans., U.S. Army Corps of Engineers, p. 4.29-4.46.

Costa, J.E., 1978, Holocene stratigraphy in flood frequency analysis: Water Resources Research, v. 14, no. 4, p. 626-632.

Costa, J.E., 1986, A history of paleoflood hydrology in the United States: Eos, Transactions of the American Geophysical Union, v. 67 , no. 17 , p. 425, 428-430.

Cowan, W.L., 1956, Estimating hydraulic roughness coefficients: Agricultural Engineering, v. 37, no. 7, p. 473-475.

Cress, Jill, Soller, David, Sayre, Roger, Comer, Patrick, and Warner, Harumi, 2010, Terrestrial ecosystems-Surficial lithology of the conterminous United States: U.S. Geological Survey Scientific Investigations Map 3126, scale 1:5,000,000, 1 sheet.

Dalrymple, Tate, and Benson, M.A., 1967, Measurement of peak discharge by the slope-area method: U.S. Geological Survey Techniques of Water-Resources Investigations, book 3, chap. A2, 12 p.
Daly, Christopher, Nielson, R.P., and Phillips, D.L., 1994, A statistical-topographic model for mapping climatological precipitation over mountainous terrain: Journal of Applied Meteorology, v. 33, no. 2, p. 140-158, accessed May 1, 2014, at http://www.prism.oregonstate.edu/.

Eash, D.A., Barnes, K.K., and Veilleux, A.G., 2013, Methods for estimating annual exceedance-probability discharges for streams in Iowa, based on data through water year 2010: U.S. Geological Survey Scientific Investigations Report 2013-5086, 63 p. with appendix, http://pubs.usgs.gov/sir/2013/5086/.

Ely, L.L., Enzel, Y., Baker, V.R., Cayan, D.R., 1993, A 5,000year record of extreme floods and climate change in the southwestern United States: Science, v. 262, p. 410-412.

Eng, Ken, Chen, Y., and Kiang, J.E., 2009, User's guide to the weighted-multiple-linear regression program (WREG version 1.0): U.S. Geological Survey Techniques and Methods, book 4, chap. A8, 21 p.

England, J.F., Jarrett, R.D., and Salas, J.D., 2003, Data-based comparisons of moments estimators using historical and paleoflood data: Journal of Hydrology, v. 278, p. 172-196.

England, J.F., Godaire, J.E., Klinger, R.E., Bauer, T.R., and Julien, P.Y., 2010, Paleohydrologic bounds and extreme flood frequency of the Upper Arkansas River, Colorado, USA: Geomorphology, v. 124, p. 1-16.

Environmental Systems Research Institute, Inc. (Esri), 2014, ArcGIS - A complete integrated system: Redlands, Calif., Esri, accessed July 28, 2014, at http:/www.esri.com/ software/arcgis/.

Enzel, Y., Ely, L.L., Martinez-Goytre, J., and Vivian, R.G., 1994, Paleofloods and a dam failure flood on the Virgin River, Utah and Arizona: Journal of Hydrology, v. 153, p. 291-315.

Farmer, W.H., Over, T.M., and Vogel, R.M., 2015, Multiple regression and inverse moments improve the characterization of the spatial scaling behavior of daily streamflows in the Southeast United States, Water Resources Research., v. 51, no 3, p. 1775-1796, doi:10.1002/ 2014WR015924.

Feaster, T.D., Gotvald, A.J., and Weaver, J.C., 2009, Magnitude and frequency of rural floods in the southeastern United States, 2006 - Volume 3, South Carolina: U.S. Geological Survey Scientific Investigations Report 2009-5043, $238 \mathrm{p}$.

Fenneman, N.M., 1931, Physiography of the Western United States: New York, McGraw-Hill, Inc., 534 p.

Frances, Felix, Salas, J.D., and Boes, D.C., 1994, Flood frequency analysis with systematic and historical or paleoflood data based on the two-parameter general extreme value models: Water Resources Research, v. 30, no. 6, p. 1,653-1,664. 
Friedman, J.M., and Lee, V.J., 2002, Extreme floods, channel change, and riparian forests along ephemeral streams: Ecological Monographs, v. 72, no. 3, p. 409-425.

Friedman, J.M., Osterkamp, W.R., Lewis, W.M., 1996, Channel narrowing and vegetation development following a Great Plains flood: Ecology, v. 77, no. 7, p. 2167-2181.

Fry, J.A., Xian, G., Jin, S., Dewitz, J.A., Homer, C.G., Yang, L., Barnes, C.A., Herold, N.D., and Wickham, J.D., 2011, Completion of the 2006 National Land Cover Database for the conterminous United States: Photogrammetric Engineering \& Remote Sensing, v. 77, no. 9, p. 858-864, accessed May 5, 2014, at http://www.mrlc.gov/nlcd2006.php.

Fulford, J.M., 1994, User's guide to SAC, a computer program for computing discharge by the slope-area method: U.S. Geological Survey Open-File Report 94-360, 31 p.

Gesch, D.B., 2007, The national elevation dataset, in Maune, D., ed., Digital elevation model technologies and applicationsThe DEM users manual, 2nd Edition: Bethesda, Maryland, American Society for Photogrammetry and Remote Sensing, p. 99-118.

Gesch, Dean, Evans, G., Mauck, J., Hutchinson, J., Carswell, W.J., Jr., 2009, The national map-Elevation: U.S. Geological Survey Fact Sheet 2009-3053, 4 p.

Gotvald, A.J., Feaster, T.D., and Weaver, J.C., 2009, Magnitude and frequency of rural floods in the southeastern United States, 2006-Volume 1, Georgia: U.S. Geological Survey Scientific Investigations Report 2009-5043, 120 p. [Also available at http://pubs.usgs.gov/sir/2009/5043/.]

Gotvald, A.J., Barth, N.A., Veilleux, A.G., and Parrett, Charles, 2012, Methods for determining magnitude and frequency of floods in California, based on data through water year 2006: U.S. Geological Survey Scientific Investigations Report 2012-5113, 38 p., 1 pl., http://pubs.usgs.gov/ sir/2012/5113/.

Gregory, K.J., 1976, Lichens and the determination of river channel capacity: Earth Surface Processes, v. 1, p. 273-285.

Griffis, V.W., and Stedinger, J.R., 2007, The use of GLS regression in regional hydrologic analyses: Journal of Hydrology, v. 344, p. 82-95, accessed March 15, 2013, at http://www.sciencedirect.com/science/article/pii/ S0022169407003848.

Grubbs, F.E., and Beck, G., 1972, Extension of sample sizes and percentage points for significance test of outlying observations: Technometrics, v. 10, p. 211-219.

Harden, T.M., O'Connor, J.E., Driscoll, D.G., and Stamm, J.F., 2011, Flood-frequency analyses from paleoflood investigations for Spring, Rapid, Boxelder, and Elk Creeks, Black Hills, western South Dakota: U.S. Geological Survey Scientific Investigations Report 2011-5131, 136 p.
Hedman, E.R., Moore, D.O., and Livingston, R.K., 1972, Selected streamflow characteristics as related to channel geometry of perennial streams in Colorado: U.S. Geological Survey Open-File Report 72-160, 24 p.

Heine, K., 2004, Flood reconstruction in the Namib Desert, Namibia and the Little Ice Age climate implications: evidence of slackwater deposits and desert soil sequences: Journal of the Geological Society of India, v. 64, p. 535-547.

Helley, E.J., and LaMarche, V.C., Jr., 1973, Historic flood information for northern California streams from geological and botanical evidence: U.S. Geological Survey Professional Paper 485-E, 16 p.

Helsel, D.R., and Hirsch, R.M., 2002, Statistical methods in water resources: U.S. Geological Survey Techniques of Water Resources Investigations, book 4, chap. A3, 522 p.

Hershfield, D.M., 1961, Rainfall frequency atlas of the United States for durations from 30 minutes to 24 hours and return periods from 1 to 100 years: U.S. Department of Commerce Weather Bureau Technical Paper No. 40, 61 p.

Hosking, J.R.M., and Wallis, J.R., 1986, Paleoflood hydrology and flood frequency analysis: Water Resources Research, v. 22 , no. 4 , p. $543-550$.

Hosman, K.J., Ely, L.L., and O'Connor, J.E., 2003, Holocene paleoflood hydrology of the lower Deschutes River, Oregon, in O'Connor, J.E., and Grant, G.E., eds., A peculiar riverGeology, geomorphology and hydrology of the Deschutes River, Oregon: American Geophysical Union Water Science and Application Series, v. 7, p. 121-146.

House, K.P., and Baker, V.R., 2001, Paleohydrology of flash flood in small desert watershed in western Arizona: Water Resources Research, v. 37, no. 6, p. 1825-1839.

House, K.P., Pearthree, P.A., and Klawon, J.E., 2002, Historical flood and paleoflood chronology of the lower Verde River, Arizona - Stratigraphic evidence and related uncertainties, in House, P.K., Webb, R.H., Baker, V.R., and Levish, D.L., eds., Ancient floods, modern hazardsPrinciples and applications of paleoflood hydrology: Washington, D.C., American Geophysical Union, Water Science and Application Series, v. 5, p. 295-310.

Hseih, Y.P., 1992, Pool size and mean age of stable soils organic carbon in cropland: Soil Science Society of America Journal, v. 56, p. 460-464.

Hseih, Y.P., 1993, Radiocarbon signatures of turnover rates in active soil organic carbon pools: Soil Science Society of America Journal, v. 57, p. 1020-1022.

Hunt, C.B., 1954, Pleistocene and recent deposits in the Denver area, Colorado: U.S. Geological Survey Bulletin 996-C, $140 \mathrm{p}$. 
Interagency Advisory Committee on Water Data, 1982, Subcommittee on Hydrology, Hydrologic Frequency Analysis Work Group, Guidelines for determining flood flow frequency: Reston, Va., Hydrology Subcommittee Bulletin 17B, 28 p., and appendixes, accessed October 5, 2015, at http://water.usgs.gov/osw/bulletin17b/dl_flow.pdf.

Interagency Advisory Committee on Water Data, 2014, Subcommittee on Hydrology, Hydrologic Frequency Analysis Work Group, Determining Flood Frequency using EMA, Frequency Asked Questions accessed July 30, 2014ロhttp://acwi.gov/ hydrology/Frequency/b17_swfaq/EMAFAQ.html.

Jacoby, Yael, Grodek, T., Enzel, Y., Porat, N., McDonald, E.V., Dahan, O., 2007, Late Holocene upper bounds of flood magnitudes and twentieth century large floods in the ungagged, hyperarid alluvial Nahal Arava, Israel: Geomorphology, v. 95, p. 274-294.

Jahns, R.E., 1947, Geologic features of the Connecticut Valley, Massachusetts, as related to recent floods: U.S. Geological Survey Water-Supply Paper 996, 158 p.

Jarrett, R.D., 1991, Paleohydrology and its value in analyzing floods and droughts, in Paulson, R.W., Chase, E.B., Roberts, R.S., and Moody, D.W., comps., National water summary 1988-89-Hydrologic events and droughts: U.S. Geological Survey Water-Supply Paper 2375, p. 105-116.

Jarrett, R.D., 2000, Paleoflood investigations for Cherry Creek Basin, eastern Colorado: 2000 Joint Conference on Water Resources Engineering and Water Resources Planning and Management [Proceedings], Minneapolis, Minn., July 30August 2, 2000, Reston, Va.: American Society of Civil Engineers, p. 1-10.

Jenny, Hans, 1941, Factors of soil formation: New York, McGraw-Hill, 281 p.

Jessup, R.W., 1960, The Stony Tableland soils of the southeastern portion of the Australian arid zone and their evolutionary history: Journal of Soil Science, v. 11, p. 88-96.

Johnson, D.L., 1990, Biomantle evolution and the redistribution of earth materials and artifacts: Soil Science, v. 149, no. 2, p. 84-102.

Kircher, J.E., Choquette, A.F., and Richter, B.D., 1985, Estimation of natural streamflow characteristics in western Colorado: U.S. Geological Survey Water-Resources Investigations Report 85-4086, 28 p.

Kochel, R.C., and Baker, V.R., 1982, Paleoflood hydrology: Science, v. 215, p. 353-361.

Kochel, R.C., and Baker, V.R., 1988, Paleoflood analysis using slackwater deposits, in Baker, V.R., Kochel, R.C., and Patton, P.C., eds., Flood geomorphology: New York, John Wiley and Sons, p. 357-376.
Levish, D.R., 2002, Paleohydrologic bounds-Nonexceedance information for flood hazard assessment, in House, P.K., Webb, R.H., Baker, V.R., Levish, D.L., eds., Ancient floods, modern hazards-Principles and applications of paleoflood hydrology: Washington, D.C., American Geophysical Union Water Science and Application Series, v. 5, p. 175-190.

Levish, D.R., and Ostenaa, D.A., 1996, Applied paleoflood hydrology in north-central Oregon, in Guidebook for field trip 2, April 19-21, 1996, Geological Society of America Cordilleran Section 92nd Annual Meeting, Portland, Oreg., $182 \mathrm{p}$.

Levish, D., Ostenaa, D., and O'Connell, D.O., 1994, A noninundation approach to paleoflood hydrology for the event based-based assessment of extreme flood hazards: American Society of Dam Safety Annual Conference Proceeding, v. 11, p. $69-82$.

Lewis, J.M., 2010, Methods for estimating the magnitude and frequency of peak streamflows for unregulated streams in Oklahoma: U.S. Geological Survey Scientific Investigations Report 2010-5137, 41 p.

Livingston, R.K., and Minges, D.R., 1987, Techniques for estimating regional flood characteristics of small rural watersheds in the plains of eastern Colorado: U.S. Geological Survey Water-Resources Investigations Report 87-4094, $72 \mathrm{p}$.

Lukas, Jeff, and Woodhouse, C., 2006, Riparian forest age structure and past hydroclimatic variability, Sand Creek Massacre National Historic Site: Eads, Colo., National Park Service, final report, 20 p., http://www.nps.gov/ sand/naturescience/upload/SAND \%2006\%20Lukas \%20Woodhouse\%20Final\%20Report.pdf.

Maas, G.S., and Macklin, M.G., 2002, The impact of recent climate change on flooding and sediment supply within a Mediterranean mountain catchment, southwestern Crete, Greece: Earth Surface Processes and Landforms, v. 27, p. 1087-1105.

Machette, M.N., 1985, Calcic soils of the southwestern United States: Geological Society of America Special Papers 203, p. 1-22.

Mahoney, J.M., and Rood, S.B., 1998, Streamflow requirements for cottonwood seedling recruitment-An integrative model: Wetlands, v. 18, p. 634-645.

Mansfield, G.R., 1938, Flood deposits of the Ohio River, January-February, 1937, a study of sedimentation, in Grover, N.C., ed., Floods of Ohio and Mississippi Rivers, January-February, 1937: U.S. Geological Survey Water Supply Paper 838, p. 693-736. 
Matthai, H.F., 1968, Magnitude and frequency of floods in the United States-Part 6B, Missouri River Basin below Sioux City, Iowa: U.S. Geological Survey Water-Supply Paper 1680, 491 p.

May, D.W., 2003, Properties of a 5500-year-old flood-plain in the Loup River Basin, Nebraska: Geomorphology, v. 56, p. 243-254.

McCain, J.F., and Jarrett, R.D., 1976, Manual for estimating flood characteristics of natural-flow streams in Colorado: Colorado Water Conservation Board Technical Manual 1, 68 p.

McFadden, L.D., McDonald, E.V., Wells, S.G., Anderson, K., Quade, J., Foreman, S.L., 1998, The vesicular layer and carbonate collars of desert soils and pavements: Formation, age, and relation to climate change: Geomorphology, v. 24, p. 101-145.

McFadden, L.D., Wells, S.G., Jercinovich, M.J., 1987, Influences of eolian and pedogenic processes on the origin and evolution of desert pavements: Geology, v. 15, p. 504-508.

McKay, L.D., Bondelid, T.R., Dewald, T.G., Johnston, C.M., Moore, R.B., and Rea, A.H., 2014, NHDPlus Version 2: User guide, 173 p., accessed July 17, 2013, at ftp://ec2-54-227-24143.compute-1.amazonaws.com/NHDplus/NHDPlusV21/ Documentation/NHDPlusV2_User_Guide.pdf.

Miller, J.F., Frederick, R.H., and Tracy, R.J., 1973, Precipitation-frequency atlas of the Western United States: National Weather Service, National Oceanic and Atmospheric Administration Atlas 2, Volume II-Wyoming, accessed August 1, 2014, at http:/www.nws.noaa.gov/oh/hdsc/ PF_documents/Atlas2_Volume2.pdf.

Miller, K.A., 2003, Peak-flow characteristics of Wyoming streams: U.S. Geological Survey Water-Resources Investigations Report 2003-4107, 79 p.

Missouri Department of Conservation, 2015, How old is that tree?: Missouri Department of Conservation, accessed February 26, 2015, at http://mdc.mo.gov/your-property/ your-trees-and-woods/backyard-tree-care/how-old-tree.

Montgomery, D.C., Peck, E.A., and Vining, G.G., 2001, Introduction to linear regression analysis ( $3 \mathrm{~d}$ ed.): New York, Wiley, $641 \mathrm{p}$.

Nebraska Department of Natural Resources, 2013, Nebraska streamgaging: Nebraska Department of Natural Resources, accessed November 8, 2013, at http:/www.dnr.nebraska.gov/ swr/streamgaging.

Nix, Steve, 2015, Estimating a tree's age-Noninvasive measurements that roughly estimate the age of a tree: About education, accessed February 26, 2015, at http://forestry.about.com/od/ silviculture/a/Estimating-A-Trees-Age.htm.
O’Connell, D.R.H., Ostenaa, D.A., Levish, D.R., and Klinger, R.E., 2002, Bayesian flood frequency analysis with paleohydrologic bound data: Water Resources Research, v. 38, no. 5, doi:10.1029/2000WR000028.

O’Connor, J.E., Ely, L.L., Wohl, E.E., Stevens, L.E., Melis, T.S., Kale, V.S., and Baker, V.R., 1994, A 4,500-year record of large floods on the Colorado River in the Grand Canyon, Arizona: Journal of Geology, v. 102, p. 1-9.

O’Connor, J.E., Atwater, B.F., Cohn, T.A., Cronin, T.M., Keith, M.K., Smith, C.G., and Mason, R.R., 2014, Assessing inundation hazards to nuclear powerplant sites using geologically extended histories of riverine floods, tsunamis, and storm surges: U.S. Geological Survey Scientific Investigations Report 2014-5207, 66 p., http://dx.doi.org/10.3133/ sir20145207.

Olson, S.A., 2014, Estimation of flood discharges at selected annual exceedance probabilities for unregulated, rural streams in Vermont, with a section on Vermont regional skew regression, by Veilleux, A.G.: U.S. Geological Survey Scientific Investigations Report 2014-5078, 27 p. plus appendixes, http://dx.doi.org/10.3133/sir20145078.

Osborn, Gerald, McCarthy, D., LaBrie, A., and Burke, R., 2015, Lichenometric dating-Science or pseudo-science?: Quaternary Research, v. 83, p. 1-12.

Patterson, J.L., 1964, Magnitude and frequency of floods in the United States_-Part 7, Lower Mississippi River Basin: U.S. Geological Survey Water-Supply Paper 1681, 636 p.

Patterson, J.L., 1965, Magnitude and frequency of floods in the United States_-Part 8, Western Gulf of Mexico basins: U.S. Geological Survey Water-Supply Paper 1682, 506 p.

Patterson, J.L., and Somers, W.P., 1966, Magnitude and frequency of floods in the United States, Part 9-Colorado River Basin: U.S. Geological Survey Water-Supply Paper 1683, 475 p.

Patton, P.C., 1987, Measuring rivers of the past-A history of fluvial paleohydrology, in Landa, E.R., and Ince, S., eds., The history of hydrology-History of geophysics, volume 3: Washington, D.C., American Geophysical Union, p. 55-67.

Patton, P.C., Baker, V.R., and Kochel, R.C., 1979, Slack water deposits - A geomorphic technique for the interpretation of fluvial paleohydrology, in Rhodes, D.D., and Williams, G.P., eds., Adjustments of the fluvial system: Dubuque, Iowa, Kendall/Hunt Publishing Company, p. 225-253.

Pelletier, J.D., Cline, M., and Delong, S.B., 2007, Desert pavement dynamics - Numerical modeling and field-based calibration: Earth Surface Processes and Landforms, v. 32, p. 1913-1927. 
Perica, Sanja, Martin, D., Pavlovic, S., Roy, I., St. Laurent, M., Trypaluk, C., Unruh, D., Yekta, M., and Bonnin, G., 2013, Precipitation-frequency atlas of the United States: National Weather Service, National Oceanic and Atmospheric Administration Atlas 14, v. 8, version 2.0; Midwestern States (Colorado, Iowa, Kansas, Michigan, Minnesota, Missouri, Nebraska, North Dakota, Oklahoma, South Dakota, Wisconsin): accessed August 1, 2014, at http:/www.nws.noaa.gov/ oh/hdsc/PF_documents/Atlas14_Volume8.pdf.

Price, C.V., Nakagaki, Naomi, and Hitt, K.J., 2010, National Water-Quality Assessment (NAWQA) area-characterization toolbox, release 1.0: U.S. Geological Survey OpenFile Report 2010-1268, accessed January 8, 2015, at http://pubs.usgs.gov/of/2010/1268.

Puseman, Kathryn, and Cummings, L.S., 2004, Separation of charcoal and organics from bulk soils samples prior to radiocarbon dating: Basin and Range Province Seismic Hazards Summit II, Reno, Nevada, May 16-19, 2004, 9 p.

Quade, Jay, 2001, Desert pavements and associated rock varnish in the Mojave Desert-How old can they be?: Geology, v. 29 , no. 9 , p. $855-858$.

Rasmussen, P.P., and Perry, C.A., 2000, Estimation of peak streamflows for unregulated rural streams in Kansas: U.S. Geological Survey Water-Resources Investigations Report 2000-4079, 33 p.

Ries, K.G., III, Steeves, P.A., Coles, J.D., Rea, A.H., and Stewart, D.W., 2004, StreamStats-A U.S. Geological Survey Web application for stream information: U.S. Geological Survey Fact Sheet 2004-3115.

Rydlund, P.H., Jr., and Densmore, B.K., 2012, Methods of practice and guidelines for using survey-grade global navigation satellite systems (GNSS) to establish vertical datum in the United States Geological Survey: U.S. Geological Survey Techniques and Methods, book 11, chap. D1, 102 p. with appendixes.

Schwarz, G.E., and Alexander, R.B., 1995, Soils data for the conterminous United States derived from the NRCS State Soil Geographic (STATSGO) data base: U.S. Geological Survey Open-File Report 95-449, accessed July 24, 2014, at http://water.usgs.gov/lookup/getspatial?ussoils.

Scott, G.R., 1960, Subdivision of the Quaternary alluvium east of the Front Range near Denver, Colorado: Geological Society of America Bulletin, v. 71, no. 10, p. 1541-1543.

Scott, G.R., 1963, Quaternary geology and geomorphic history of the Kassler Quadrangle Colorado: U.S. Geological Survey Professional Paper 421-A, 70 p., 1 sheet, scale 1:24,000.
Scott, M.L., Auble, G.T., Friedman, J.M., 1997, Flood dependency of cottonwood establishment along the Missouri River, Montana, USA: Ecological Applications, v. 7, no. 2, p. 677-690.

Sheffer, N.A., Rico, M., Enzel, Y., Benito, G., and Grodek, T., 2008, The paleoflood record of the Gardon River, FranceA comparison with the extreme 2002 flood event: Geomorphology, v. 98, p. 71-83.

Soenksen, P.J., Miller, L.D., Sharpe, J.B., and Watton, J.R., 1999, Peak-flow frequency relations and evaluation of the peak-flow gaging network in Nebraska: U.S. Geological Survey Water-Resources Investigations Report 99-4032, $48 \mathrm{p}$.

Southard, R.E., and Veilleux, A.G., 2014, Methods for estimating annual exceedance-probability discharges and largest recorded floods for unregulated streams in rural Missouri: U.S. Geological Survey Scientific Investigations Report 2014-5165, 39 p., http://dx.doi.org/10.3133/ sir20145165.

Stedinger, J.R., and Baker, V.R., 1987, Surface water hydrology-Historical and paleoflood Information: Review of Geophysics, v. 25, no. 2, p. 119-124.

Stedinger, J.R., and Cohn, T.A., 1986, Flood frequency analysis with historical and paleoflood information: Water Resources Research, v. 22, no. 5, p. 785-793.

Stedinger, J.R., and Tasker, G.D., 1985, Regional hydrologic analysis I-Ordinary, weighted, and generalized leastsquares compared: American Geophysical Union, Water Resources Research, v. 21, no. 9, p. 1421-1432.

Stuvier, Minze, and Becker, B., 1986, High-precision decadal calibration of the decadal time scale A.D. 1950-2500 B.C.: Radiocarbon, v. 28, p. 863-910.

Stuvier, Minze, and Pearson, G.W., 1986, High-precision calibration of the radiocarbon time scale, A.D. 1950-50 B.C.: Radiocarbon, v. 28, no. 2b, p. 803-838.

Tasker, G.D., and Stedinger, J.R., 1986, Regional skew with weighted LS regression: American Society of Civil Engineers Journal of Water Resources Planning and Management, v. 112, no. 2, p. 225-237.

Tasker, G.D., and Stedinger, J.R., 1989, An operational GLS model for hydrologic regression: Journal of Hydrology, v. 111 , p. 361-375.

U.S. Army Corps of Engineers, 2010a, HEC-RAS River Analysis System user's manual, version 4.1: U.S. Army Corps of Engineers, 790 p.

U.S. Army Corps of Engineers, 2010b, HEC-RAS River Analysis System hydraulic reference manual, version 4.1: U.S. Army Corps of Engineers, 417 p. 
U.S. Geological Survey (USGS), 2013a, National water information system-Mapper: U.S. Geological Survey Web site, accessed August 9, 2013, at http://maps.waterdata.usgs.gov/ mapper/.

U.S. Geological Survey (USGS), 2013b, National water information system - Web interface-USGS water data for the Nation: U.S. Geological Survey Web site, accessed November 8, 2013, at http://waterdata.usgs.gov/nwis/.

U.S. Geological Survey (USGS), 2015, The StreamStats program: U.S. Geological Survey Web site, accessed February 27, 2015, at http://water.usgs.gov/osw/streamstats/.

Vaill, J.E., 1999, Analysis of the magnitude and frequency of floods in Colorado: U.S. Geological Survey WaterResources Investigations Report 99-4190, 35 p.

Veilleux, A.G., 2009, Bayesian GLS regression for regionalization of hydrologic statistics, floods and bulletin 17 skew: Cornell University Master of Science Thesis, 155 p., accessed October 2, 2015, at https://ecommons.cornell.edu/bitstream/ handle/1813/13819/Veilleux,\%20Andrea.pdf?sequence=1.

Veilleux, A.G., Cohn, T.A., Flynn, K.M., Mason, R.R., Jr., and Hummel, P.R., 2014, Estimating magnitude and frequency of floods using the PeakFQ 7.0 program: U.S. Geological Survey Fact Sheet 2013-3108, 2 p.

Venables, W.N., and Smith, D.M., 2014, An introduction to R, Version 3.1.2, accessed February 27, 2015, at http://www.r-project.org/.

Verdi, R. J., and Dixon, J. F., 2011, Magnitude and frequency of floods for rural streams in Florida, 2006: U.S. Geological Survey Scientific Investigations Report 2011-5034, 69 p., 1 pl. at http://pubs.usgs.gov/sir/2011/5034/.
Vogel, R.M., and Sankarasubramanian, A., 2000, Spatial scaling properties of annual streamflow in the United States: Hydrological Sciences Journal, v. 45, no. 3, p. 465-476, doi:10.1080/02626660009492342.

Waltemeyer, S.D., 2008, Analysis of the magnitude and frequency of peak discharge and maximum observed peak discharge in New Mexico and surrounding areas: U.S. Geological Survey Scientific Investigations Report 2008-5119, $105 \mathrm{p}$.

Weaver, J.C., Feaster, T.D., and Gotvald, A.J., 2009, Magnitude and frequency of rural floods in the southeastern United States, through 2006 - Volume 2, North Carolina: U.S. Geological Survey Scientific Investigations Report 2009-5158, 111 p. [Also available at: http://pubs.usgs.gov/sir/2009/5158/.]

Webb, R.H., Blainey, J.B., and Hyndman, D.W., 2002, Paleoflood hydrology of the Paria River, southern Utah and northern Arizona, USA, in House, P.K., Webb, R.H., Baker, V.R., and Levish, D.L., eds., Ancient floods, modern hazards - Principles and applications of paleoflood hydrology: Washington, D.C., American Geophysical Union's Water Science and Application Series, v. 5, p. 295-310.

Williams, S.H., and Zimbelman, J.R., 1994, Desert pavement evolution-An example of the role of sheetflow: Journal of Geology, v. 102, p. 243-248.

Wohl, E.E., Webb, R.H., Baker, V.R., and Pickup, G., 1994, Sedimentary flood records in the bedrock canyons of rivers in the monsoonal region of Australia: Water Resources Papers, v. 107, 102 p.

Wolock, D.M., 1997, STATSGO soil characteristics for the conterminous United States: U.S. Geological Survey Open-File Report 97-656, accessed July 24, 2014, at http://water.usgs.gov/lookup/getspatial?muid. 
Appendixes 



\section{Appendix 1. Example Paleoflood Investigation and Computation of Streamgage Peak-Streamflow Frequency at 06758700 Middle Bijou Creek Tributary near Deer Trail, Colo.}

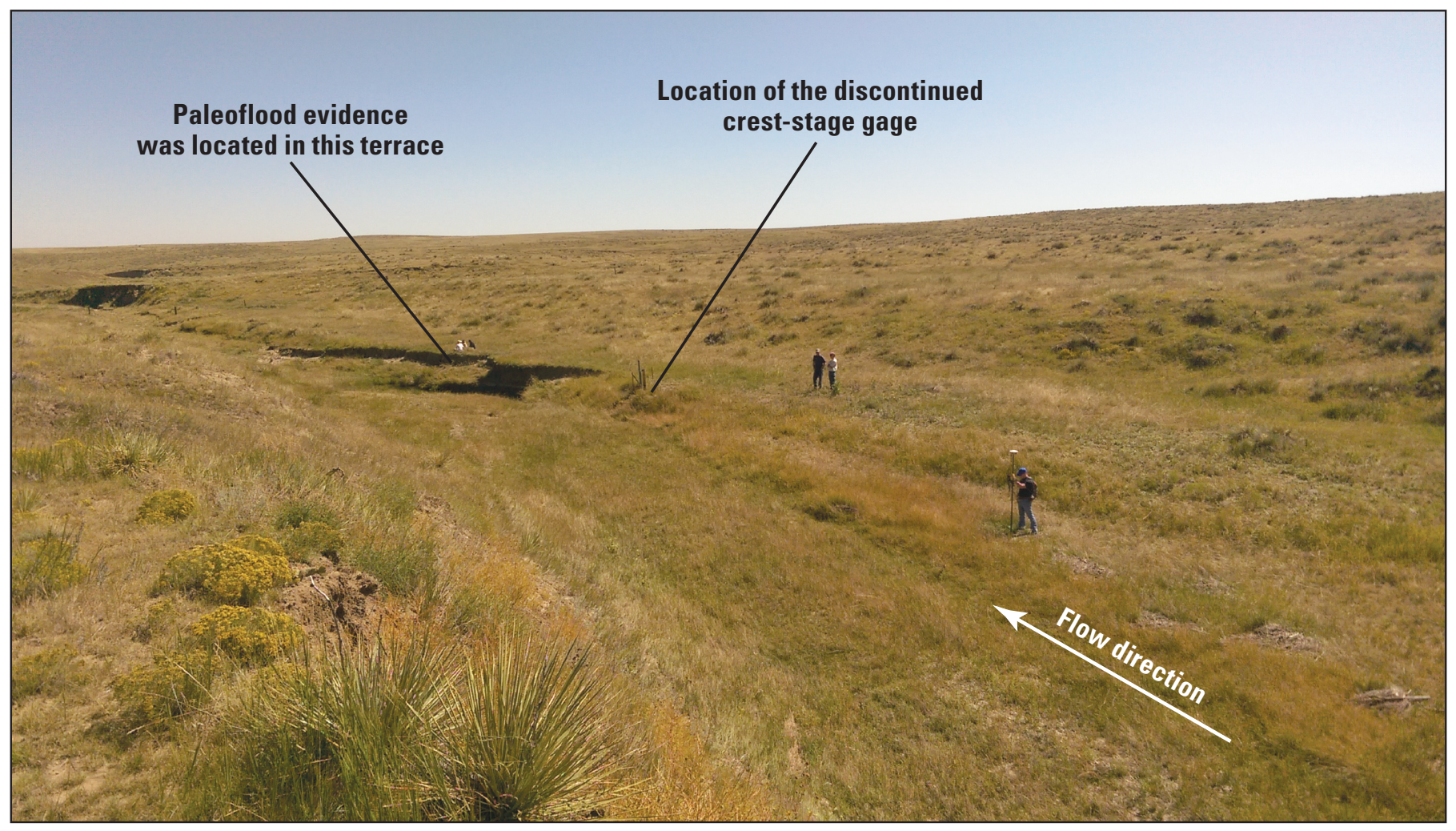

Figure 1-1. Looking downstream toward the discontinued crest-stage gage at 06758700 Middle Bijou Creek Tributary near Deer Trail, Colo.; paleoflood evidence was identified in a terrace along the right bank approximately 100 feet downstream from the streamgage. Photograph by Michael Kohn, USGS. 


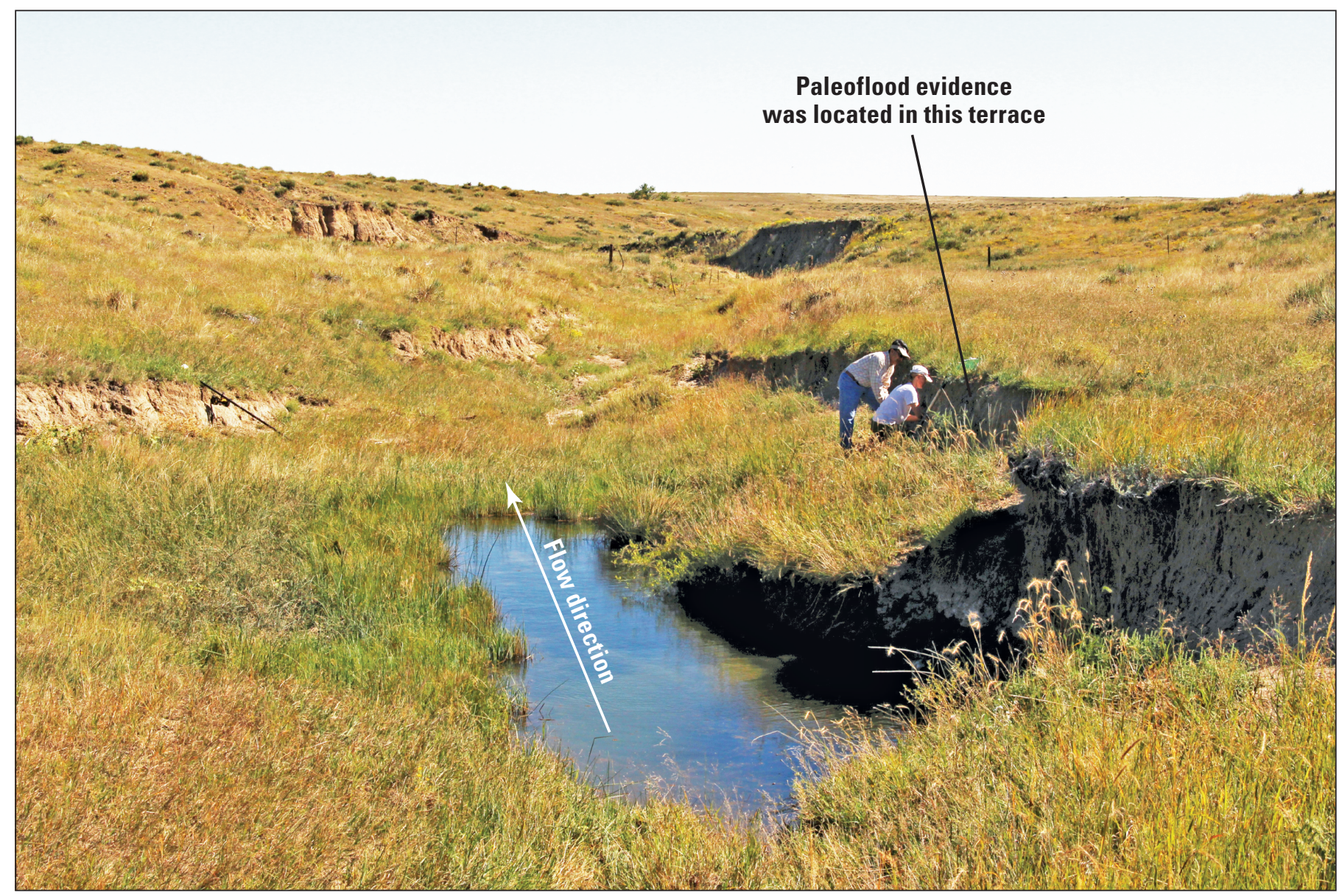

Figure 1-2. Looking downstream at 06758700 Middle Bijou Creek Tributary near Deer Trail, Colo.; paleoflood evidence was identified in a terrace along the right bank approximately 100 feet downstream from the streamgage; photograph taken from the discontinued crest-stage gage. Photograph by Michael Stevens, USGS. 


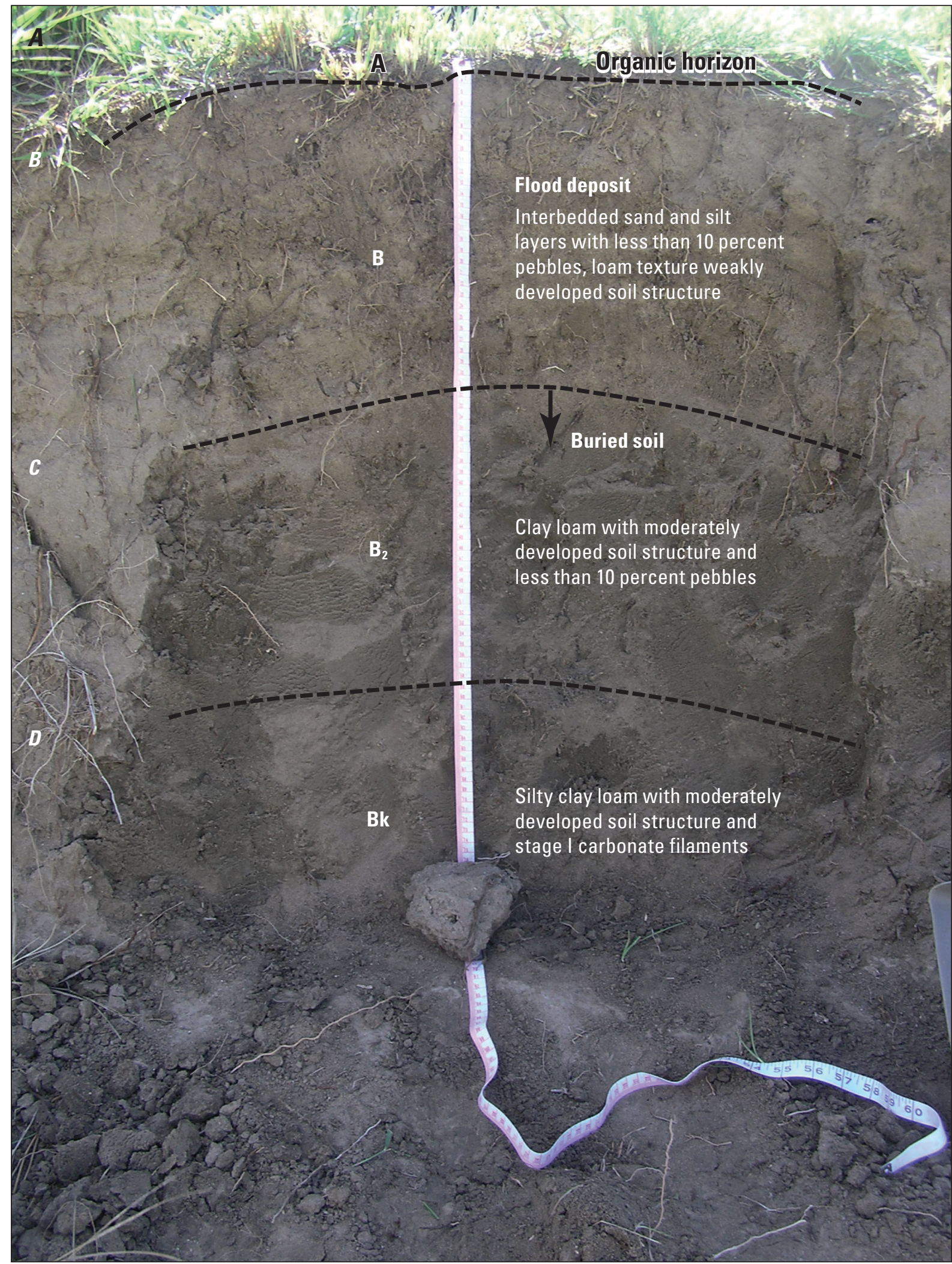

Figure 1-3. Four horizons are present in this terrace exposure; $A$, an organic rich $\mathrm{A}$ horizon, $B$, a $\mathrm{B}$ horizon with sand and silt interbeds, $C$, a more developed $B_{2}$ horizon, and $D$, and older Bk horizon at the bottom. Photograph by Jeanne Godaire, Bureau of Reclamation. 


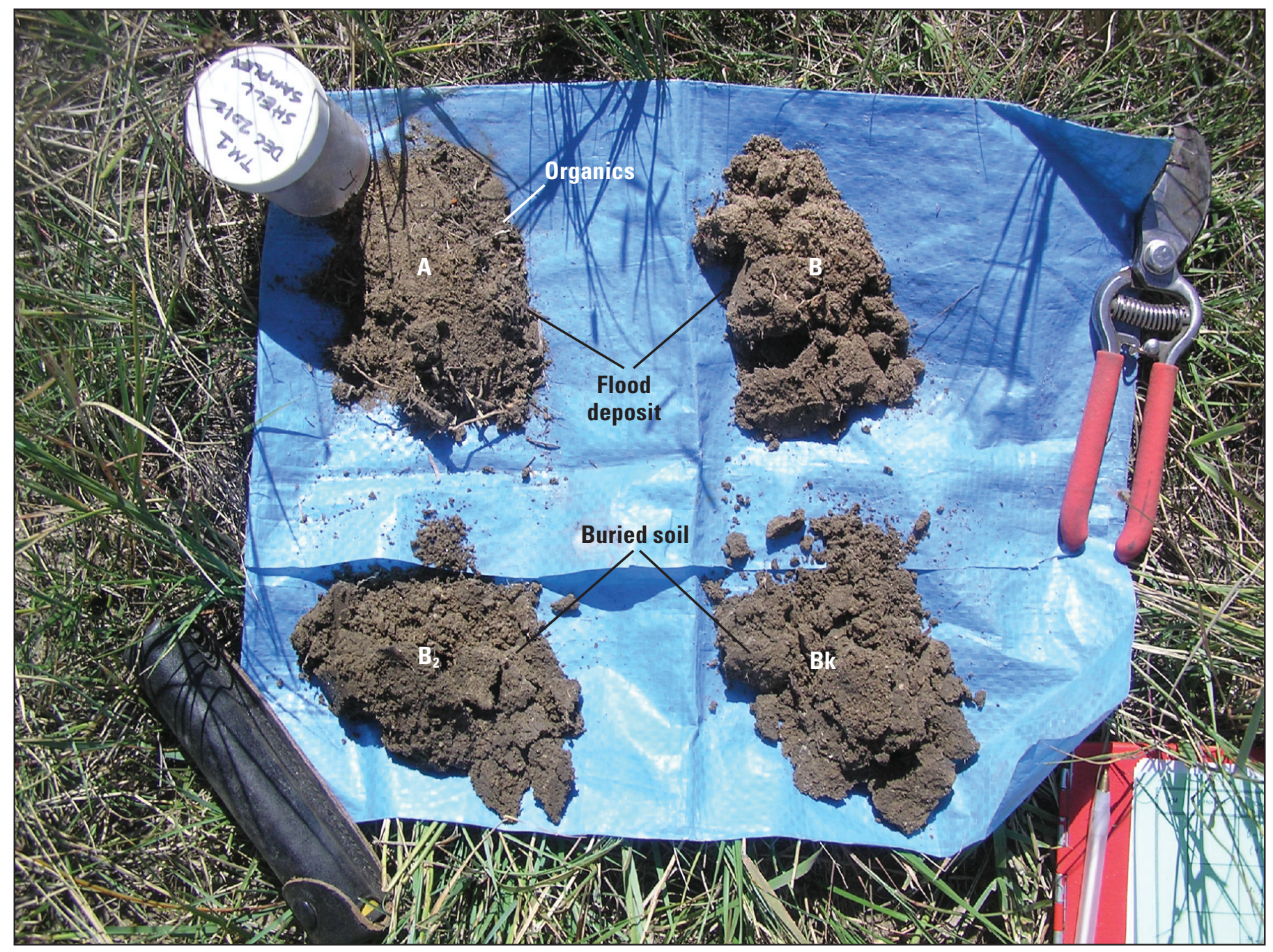

Figure 1-4. These soil samples correspond to the soil horizons in figure 1-3. The soil structure goes from single grained and weakly granular in the A horizon to weak to moderate subangular blocky in the Bk horizon. Photograph by Jeanne Godaire, Bureau of Reclamation. 


\section{Cross Section X0}

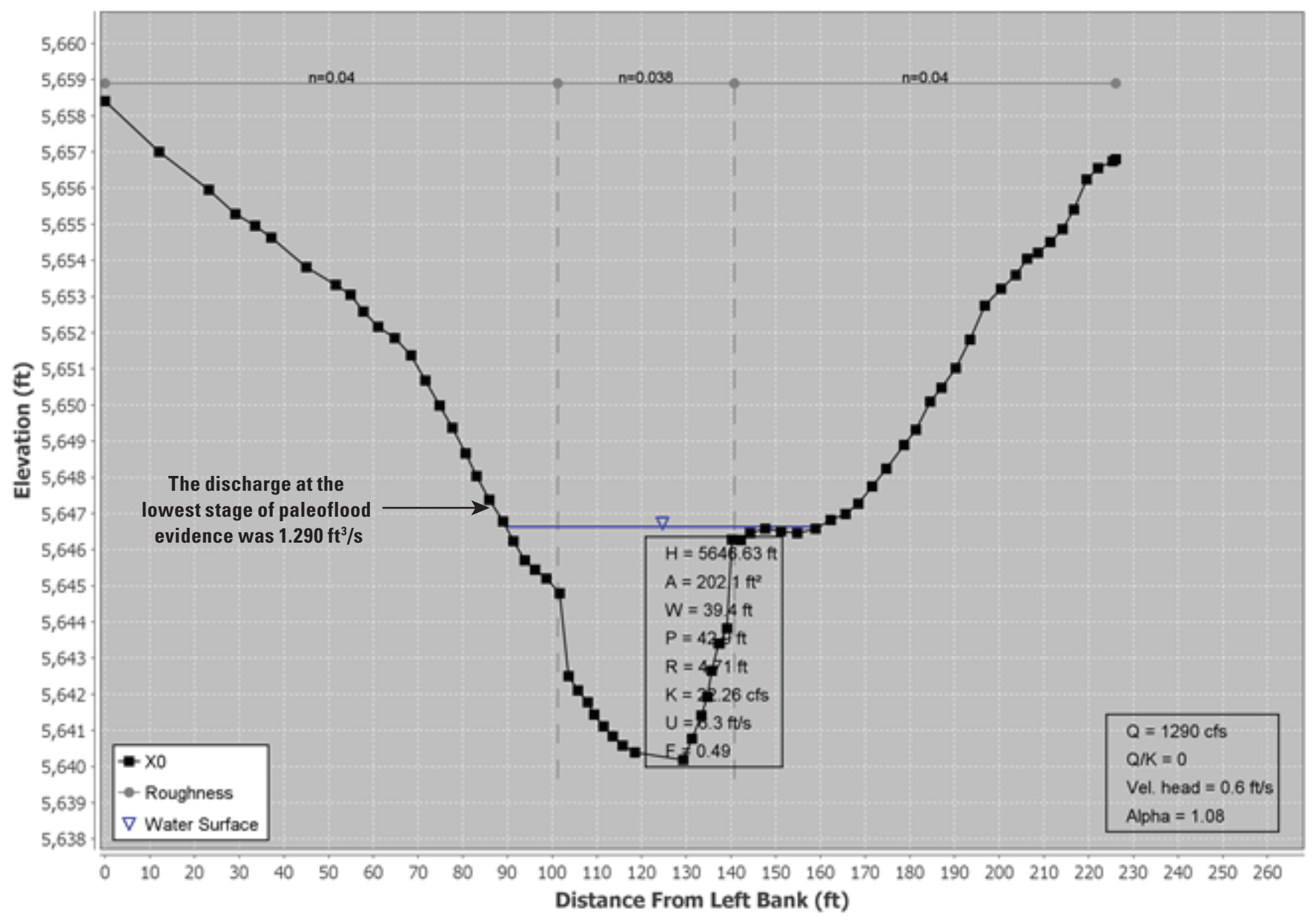

Figure 1-5. Lower limit discharge model output from Slope-Area Computation Graphical User Interface at the cross section where the terrace with paleoflood evidence was located; the lower limit discharge of 1,290 cubic feet per second ( $\mathrm{ft}^{3} / \mathrm{s}$ or cfs) was computed by using the lowest stage of the paleoflood evidence, increasing the Manning roughness coefficient ( $\mathrm{n}$ ) by 20 percent, and decreasing the channel slope by 25 percent. $H$, water-surface elevation; $A$, cross-sectional area; $W$, width; $P$, wetted perimeter; $R$, hydraulic radius; $\mathrm{K}$, conveyance; U, velocity; F, Froude number; $\mathrm{Q}$, discharge; Vel. head, velocity head; n, Manning roughness coefficient; ft, foot; alpha, kinetic energy flux correction. 


\section{Cross Section X0}

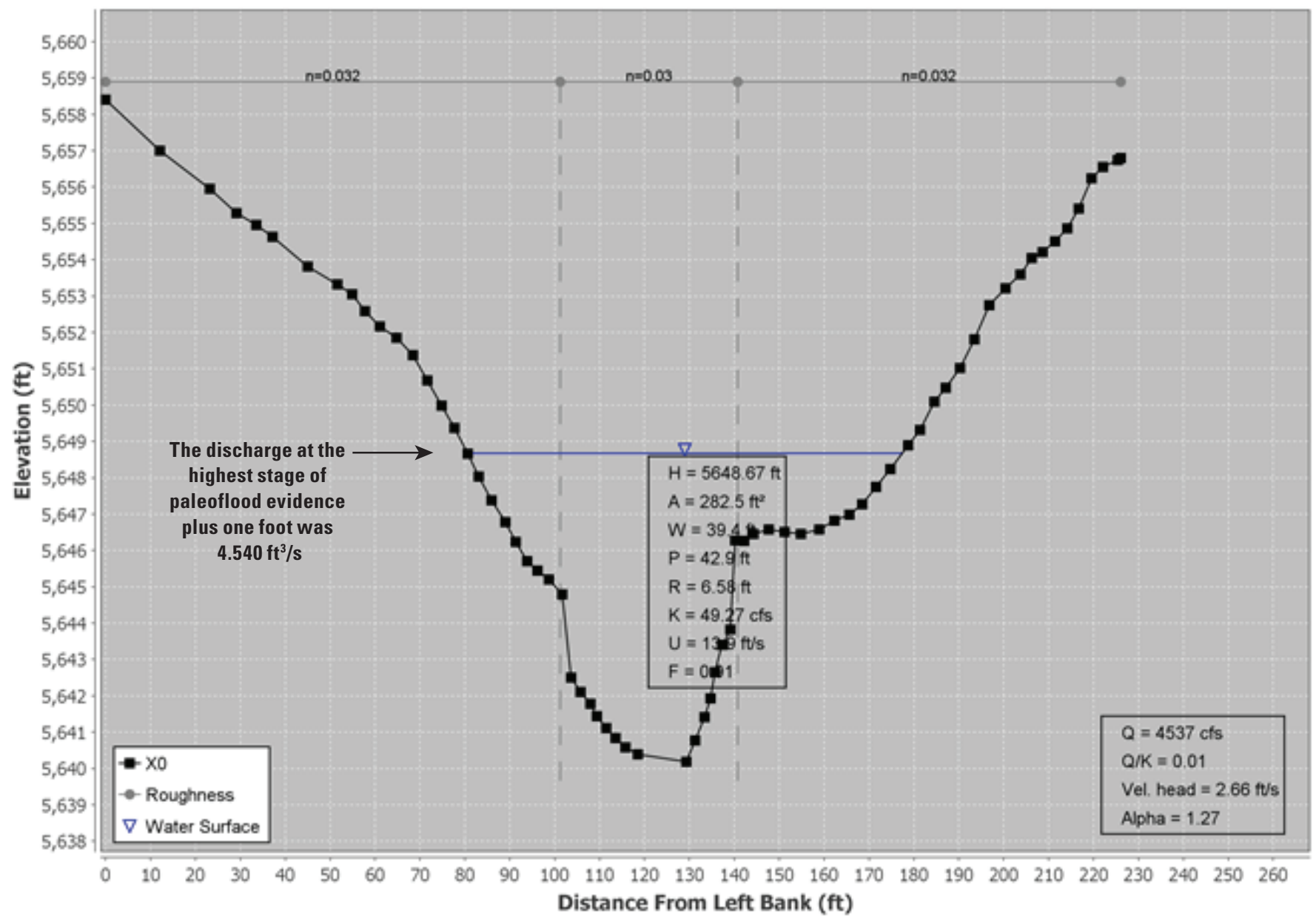

Figure 1-6. The upper limit discharge model output from Slope-Area Computation Graphical User Interface at the cross section where the terrace with paleoflood evidence was located; the upper limit discharge of 4,540 cubic feet per second (ft $3 / \mathrm{s}$ or cfs) was computed by using the highest stage of the paleoflood evidence plus 1 foot ( 3 feet for non-exceedance bounds), decreasing the Manning roughness coefficient (n) by 20 percent, and increasing the channel slope by 25 percent. $H$, water-surface elevation; $A$, cross-sectional area; $W$, width; P, wetted perimeter; $R$, hydraulic radius; $K$, conveyance; $U$, velocity; F, Froude number; 0 , discharge; Vel. head, velocity head; $n$, Manning roughness coefficient; $\mathrm{ft}$, foot; alpha, kinetic energy flux correction. 


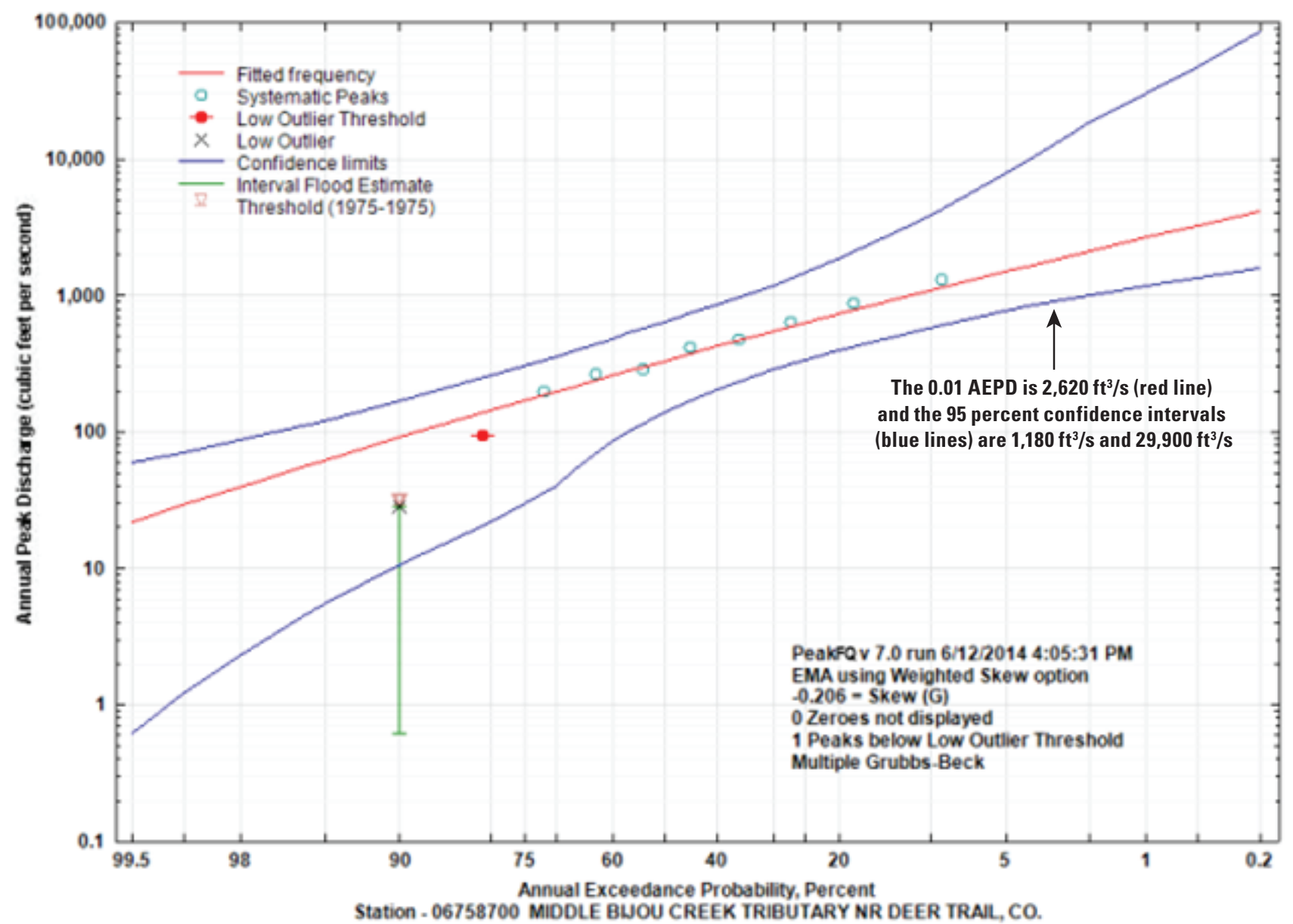

Figure 1-7. The flood-frequency curve for 06758700 Middle Bijou Creek Tributary near Deer Trail, Colo. prior to the inclusion of any paleoflood data; the 0.01 annual exceedance-probability discharge is 2,620 cubic feet per second ( $\mathrm{ft}^{3} / \mathrm{s}$ ) (red line) and the 95 percent confidence intervals (blue lines) are 1,180 ft $3 / \mathrm{s}$ and 29,900 fts. AEPD, annual exceedance-probability discharge; EMA, Expected Moments Algorithm; Skew (G), weighted skew. 


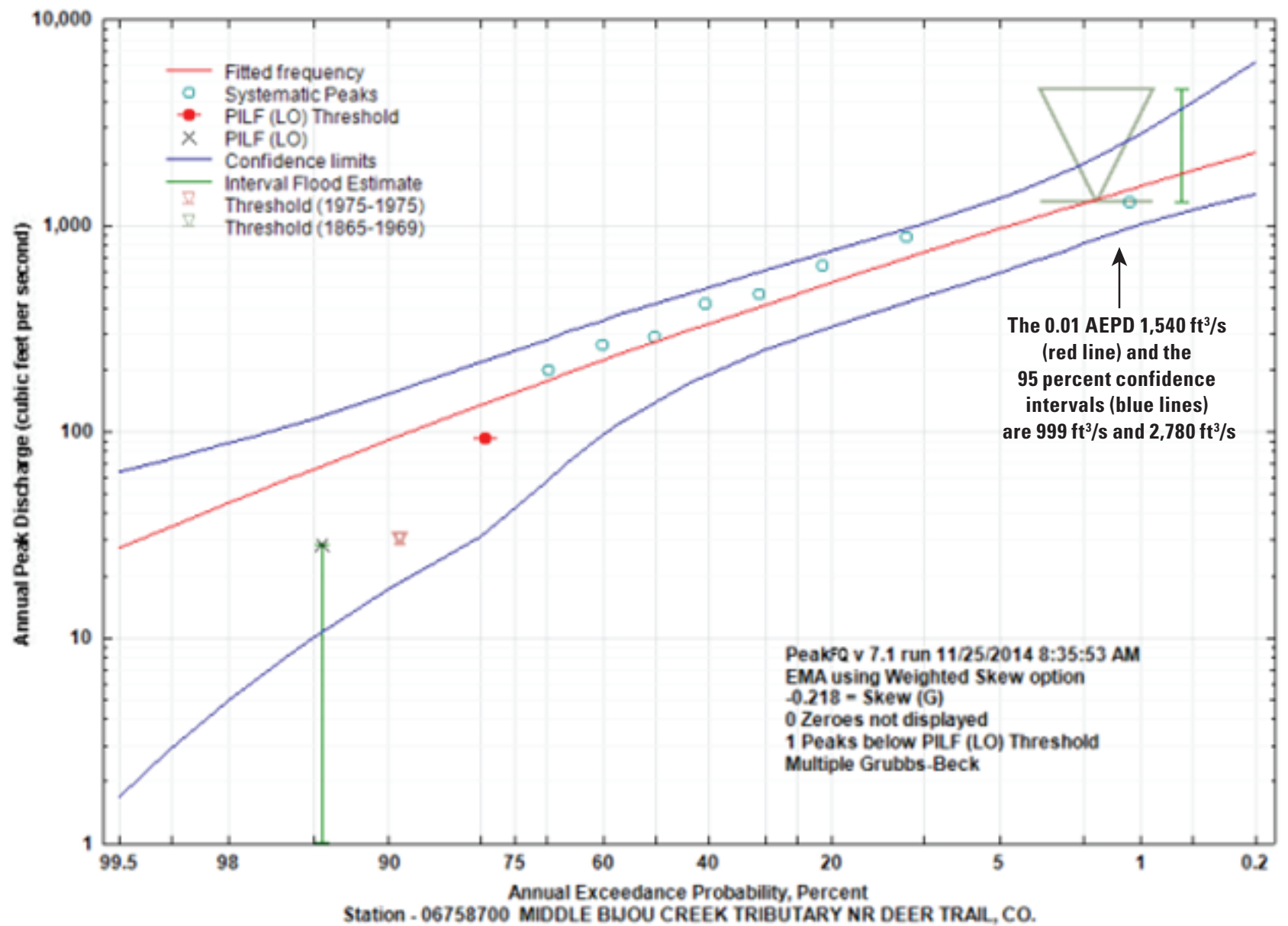

Figure 1-8. The flood-frequency curve for 06758700 Middle Bijou Creek Tributary near Deer Trail, Colo. with a paleoflood that has a lower limit discharge of 1,290 cubic feet per second $\left(\mathrm{ft}^{3} / \mathrm{s}\right)$, upper limit discharge of $4,540 \mathrm{ft}^{3} / \mathrm{s}$, and age of $150 \mathrm{years}$; the 0.01 annual exceedance-probability discharge has been reduced by 41 percent to 1,540 $\mathrm{ft}^{3} / \mathrm{s}$ (red line) and the $95 \mathrm{percent}$ confidence intervals (blue lines) are $999 \mathrm{ft}^{3} / \mathrm{s}$ and 2,780 $\mathrm{ft}^{3} / \mathrm{s}$. AEPD, annual exceedance-probability discharge; PILF (LO), low outlier; EMA, Expected Moments Algorithm; Skew (G), weighted skew. 


\section{Appendix 2. Paleoflood Data}

Table 2-1. Summary of the paleoflood data collected and applied at 44 streamgages used in the regression analysis of natural streams in eastern Colorado, 2015.

[USGS, U.S. Geological Survey; $Q$, discharge; $Q_{\text {low }}$ low uncertainty discharge; $Q_{\text {high }}$, high uncertainty discharge; HEC-RAS, Hydrologic Engineering Center River Analysis System; SAC, Slope-Area Computation, Nebr., Nebraska; Colo., Colorado; Wyo., Wyoming; Kans., Kansas; Do., repeat from row above; Okla., Oklahoma; NA, not applicable; N. Mex., New Mexico]

\begin{tabular}{|c|c|c|c|c|c|c|c|c|c|}
\hline \multirow{2}{*}{$\begin{array}{l}\text { USGS } \\
\text { stream- } \\
\text { gage } \\
\text { number }\end{array}$} & \multirow[b]{2}{*}{ Streamgage name } & \multirow[b]{2}{*}{$\begin{array}{c}\text { Date } \\
\text { collected }\end{array}$} & \multirow[b]{2}{*}{$\begin{array}{l}\text { Data } \\
\text { type }\end{array}$} & \multicolumn{3}{|c|}{$\begin{array}{l}\text { Annual exceedance- } \\
\text { probability discharge } \\
\text { (percent) }\end{array}$} & \multirow{2}{*}{$\begin{array}{c}\text { Age, } \\
\text { in years } \\
\text { from date } \\
\text { collected }\end{array}$} & \multirow{2}{*}{$\begin{array}{c}\text { Age } \\
\text { uncer- } \\
\text { tainty, } \\
\text { in years }\end{array}$} & \multirow[b]{2}{*}{ Method } \\
\hline & & & & $\begin{array}{c}0 \\
\text { (in cubic } \\
\text { feet per } \\
\text { second) }\end{array}$ & $\begin{array}{c}Q_{\text {low }} \\
\text { (in cubic } \\
\text { feet per } \\
\text { second) }\end{array}$ & $\begin{array}{c}Q_{\text {high }} \\
\text { (in cubic } \\
\text { feet per } \\
\text { second) }\end{array}$ & & & \\
\hline 06687000 & Blue Creek near Lewellen, Nebr. & 2014 & $\begin{array}{l}\text { Non-exceedance } \\
\text { bound }\end{array}$ & 3,970 & 2,860 & 18,700 & 350 & \pm 150 & HEC-RAS \\
\hline 06719500 & Clear Creek near Golden, Colo. & 2002 & Paleoflood & 50,100 & 37,600 & 62,600 & 6,000 & Unknown & $\begin{array}{l}\text { Flow } \\
\text { competence }\end{array}$ \\
\hline 06755960 & $\begin{array}{l}\text { Crow Creek at 19th Street, at } \\
\text { Cheyenne, Wyo. }\end{array}$ & 2014 & $\begin{array}{l}\text { Non-exceedance } \\
\text { bound }\end{array}$ & 11,000 & 7,140 & 22,700 & 125 & \pm 25 & HEC-RAS \\
\hline 06758200 & Kiowa Creek at Kiowa, Colo. & 2014 & $\begin{array}{l}\text { Non-exceedance } \\
\text { bound }\end{array}$ & 19,700 & 19,700 & 19,700 & 49 & 0 & Gage record \\
\hline 06763500 & Lodgepole Creek at Ralton, Nebr. & 2014 & $\begin{array}{l}\text { Non-exceedance } \\
\text { bound }\end{array}$ & 18,500 & 13,400 & 48,600 & 300 & \pm 200 & HEC-RAS \\
\hline 06831500 & Frenchman Creek near Imperial, Nebr. & 2014 & $\begin{array}{l}\text { Non-exceedance } \\
\text { bound }\end{array}$ & 4,000 & 3,150 & 21,900 & 200 & \pm 100 & HEC-RAS \\
\hline 06835100 & Bobtail Creek near Palisade, Nebr. & 2014 & $\begin{array}{l}\text { Non-exceedance } \\
\text { bound }\end{array}$ & 15,200 & 15,200 & 105,000 & 350 & \pm 150 & HEC-RAS \\
\hline 06844800 & $\begin{array}{l}\text { South Fork Sappa Creek Tributary near } \\
\text { Goodland, Kans. }\end{array}$ & 2014 & $\begin{array}{l}\text { Non-exceedance } \\
\text { bound }\end{array}$ & 3,450 & 3,450 & 3,450 & 57 & 0 & Gage record \\
\hline 06858500 & $\begin{array}{l}\text { North Fork Smoky Hill River near } \\
\text { McAllaster, Kans. }\end{array}$ & 2014 & $\begin{array}{l}\text { Non-exceedance } \\
\text { bound }\end{array}$ & 50,000 & 32,700 & 129,000 & 300 & \pm 200 & HEC-RAS \\
\hline 07099215 & Turkey Creek near Fountain, Colo. & 2014 & $\begin{array}{l}\text { Non-exceedance } \\
\text { bound }\end{array}$ & 1,650 & 1,560 & 2,480 & 150 & \pm 50 & SAC \\
\hline 07103977 & $\begin{array}{l}\text { Cottonwood Creek at Cowpoke Road at } \\
\text { Colorado Springs, Colo. }\end{array}$ & 2014 & $\begin{array}{l}\text { Non-exceedance } \\
\text { bound }\end{array}$ & 6,100 & 5,500 & 9,630 & 300 & \pm 200 & HEC-RAS \\
\hline \multirow[t]{2}{*}{07105000} & Bear Creek near Colorado Springs, Colo. & 2014 & $\begin{array}{l}\text { Non-exceedance } \\
\text { bound }\end{array}$ & 1,600 & 918 & 8,810 & 300 & \pm 200 & SAC \\
\hline & Do. & 2014 & $\begin{array}{l}\text { Non-exceedance } \\
\text { bound }\end{array}$ & 4,060 & 3,000 & 13,700 & 750 & \pm 250 & SAC \\
\hline 07105490 & $\begin{array}{l}\text { Cheyenne Creek at Evans Avenue at } \\
\text { Colorado Springs, Colo. }\end{array}$ & 2014 & $\begin{array}{l}\text { Non-exceedance } \\
\text { bound }\end{array}$ & 3,000 & 2,700 & 6,800 & 125 & \pm 75 & HEC-RAS \\
\hline 07105940 & $\begin{array}{l}\text { Little Fountain Creek near Fountain, } \\
\text { Colo. }\end{array}$ & 2014 & $\begin{array}{l}\text { Non-exceedance } \\
\text { bound }\end{array}$ & 18,000 & 7,140 & 41,600 & 150 & \pm 50 & SAC \\
\hline \multirow[t]{2}{*}{07107500} & St. Charles River at Burnt Mill, Colo. & 2014 & $\begin{array}{l}\text { Non-exceedance } \\
\text { bound }\end{array}$ & 21,800 & 21,800 & 21,800 & 91 & 0 & Gage record \\
\hline & Do. & 2014 & $\begin{array}{l}\text { Non-exceedance } \\
\text { bound }\end{array}$ & 24,500 & 21,800 & 45,400 & 150 & \pm 50 & HEC-RAS \\
\hline
\end{tabular}


Table 2-1. Summary of the paleoflood data collected and applied at 44 streamgages used in the regression analysis of natural streams in eastern Colorado, 2015. - Continued

[USGS, U.S. Geological Survey; $Q$, discharge; $Q_{\text {low }}$ low uncertainty discharge; $Q_{\text {high }}$, high uncertainty discharge; $\mathrm{ft}^{3} / \mathrm{s}$, cubic foot per second; HEC-RAS, Hydrologic Engineering Center River Analysis System; SAC, Slope-Area Computation, Nebr., Nebraska; Colo., Colorado; Wyo., Wyoming; Kans., Kansas; Do., repeat from row above; Okla., Oklahoma; NA, not applicable; N. Mex., New Mexico]

\begin{tabular}{|c|c|c|c|c|c|c|c|c|c|}
\hline \multirow{2}{*}{$\begin{array}{l}\text { USGS } \\
\text { stream- } \\
\text { gage } \\
\text { number }\end{array}$} & \multirow[b]{2}{*}{ Streamgage name } & \multirow[b]{2}{*}{$\begin{array}{l}\text { Date } \\
\text { collected }\end{array}$} & \multirow[b]{2}{*}{$\begin{array}{l}\text { Data } \\
\text { type }\end{array}$} & \multicolumn{3}{|c|}{$\begin{array}{c}\text { Annual exceedance- } \\
\text { probability discharge } \\
\text { (percent) }\end{array}$} & \multirow{2}{*}{$\begin{array}{c}\text { Age, } \\
\text { in years } \\
\text { from date } \\
\text { collected }\end{array}$} & \multirow{2}{*}{$\begin{array}{c}\text { Age } \\
\text { uncer- } \\
\text { tainty, } \\
\text { in years }\end{array}$} & \multirow[b]{2}{*}{ Method } \\
\hline & & & & $\begin{array}{c}0 \\
\text { (in cubic } \\
\text { feet per } \\
\text { second) }\end{array}$ & $\begin{array}{c}\boldsymbol{O}_{\text {low }} \\
\text { (in cubic } \\
\text { feet per } \\
\text { second) }\end{array}$ & $\begin{array}{c}\boldsymbol{Q}_{\text {high }} \\
\text { (in cubic } \\
\text { feet per } \\
\text { second) }\end{array}$ & & & \\
\hline 07120620 & Big Arroyo near Thatcher, Colo. & 2014 & $\begin{array}{l}\text { Non-exceedance } \\
\text { bound }\end{array}$ & 5,120 & 4,260 & 15,600 & 750 & \pm 250 & HEC-RAS \\
\hline 07125100 & Frijole Creek near Alfalfa, Colo. & 2014 & $\begin{array}{l}\text { Non-exceedance } \\
\text { bound }\end{array}$ & 28,400 & 28,400 & 62,000 & 200 & \pm 100 & HEC-RAS \\
\hline \multirow{3}{*}{07125500} & Do. & 2014 & Paleoflood & 6,540 & 5,420 & 7,660 & 32 & 0 & HEC-RAS \\
\hline & San Francisco Creek near Alfalfa, Colo. & 2014 & $\begin{array}{l}\text { Non-exceedance } \\
\text { bound }\end{array}$ & 26,300 & 26,300 & 26,300 & 60 & 0 & Gage record \\
\hline & Do. & 2014 & $\begin{array}{l}\text { Non-exceedance } \\
\text { bound }\end{array}$ & 26,300 & 26,300 & 55,500 & 2,000 & $\pm 1,000$ & HEC-RAS \\
\hline 07126390 & $\begin{array}{l}\text { Lockwood Canyon Creek near } \\
\text { Thatcher, Colo. }\end{array}$ & 2014 & $\begin{array}{l}\text { Non-exceedance } \\
\text { bound }\end{array}$ & 1,110 & 1,110 & 1,110 & 75 & \pm 25 & Gage record \\
\hline 07126415 & $\begin{array}{l}\text { Red Rock Canyon Creek at Mouth near } \\
\text { Thatcher, Colo. }\end{array}$ & 2014 & $\begin{array}{l}\text { Non-exceedance } \\
\text { bound }\end{array}$ & 21,200 & 15,700 & 45,300 & 150 & \pm 50 & HEC-RAS \\
\hline 07126480 & $\begin{array}{l}\text { Bent Canyon Creek at Mouth near } \\
\text { Timpas, Colo. }\end{array}$ & 2014 & $\begin{array}{l}\text { Non-exceedance } \\
\text { bound }\end{array}$ & 7,530 & 5,470 & 25,000 & 150 & \pm 50 & SAC \\
\hline 07134100 & Big Sandy Creek near Lamar, Colo. & 2014 & $\begin{array}{l}\text { Non-exceedance } \\
\text { bound }\end{array}$ & 3,600 & 3,600 & 3,600 & 80 & \pm 10 & Gage record \\
\hline 07135000 & Two Butte Creek near Holly, Colo. & 2014 & $\begin{array}{l}\text { Non-exceedance } \\
\text { bound }\end{array}$ & 2,820 & 2,090 & 10,100 & 70 & \pm 10 & $\mathrm{SAC}$ \\
\hline 07138600 & $\begin{array}{l}\text { White Woman Creek Tributary near } \\
\text { Selkirk, Kans. }\end{array}$ & 2014 & $\begin{array}{l}\text { Non-exceedance } \\
\text { bound }\end{array}$ & 1,870 & 1,440 & 4,790 & 500 & \pm 100 & SAC \\
\hline \multirow[t]{2}{*}{07156600} & $\begin{array}{l}\text { Cimarron River Tributary near } \\
\text { Moscow, Kans. }\end{array}$ & 2014 & $\begin{array}{l}\text { Non-exceedance } \\
\text { bound }\end{array}$ & 3,900 & 3,900 & 3,900 & 24 & 0 & Gage record \\
\hline & Do. & 2014 & $\begin{array}{l}\text { Non-exceedance } \\
\text { bound }\end{array}$ & 9,620 & 7,100 & NA & 2,000 & $\pm 1,000$ & $\mathrm{SAC}$ \\
\hline 07201200 & $\begin{array}{l}\text { Chicorica Creek Tributary near Raton, } \\
\text { N. Mex. }\end{array}$ & 2014 & $\begin{array}{l}\text { Non-exceedance } \\
\text { bound }\end{array}$ & 3,500 & 2,580 & 9,800 & 350 & \pm 150 & $\mathrm{SAC}$ \\
\hline 07201450 & $\begin{array}{l}\text { Green Mountain Arroyo near Raton, N. } \\
\text { Mex. }\end{array}$ & 2014 & Paleoflood & 15,000 & 10,200 & 28,100 & 100 & \pm 40 & $\mathrm{SAC}$ \\
\hline \multirow[t]{2}{*}{07203600} & $\begin{array}{l}\text { Rio Del Plano Tributary near Taylor } \\
\text { Springs, N. Mex. }\end{array}$ & 2014 & $\begin{array}{l}\text { Non-exceedance } \\
\text { bound }\end{array}$ & 2,820 & 1,820 & 5,600 & 500 & \pm 100 & $\mathrm{r}$ \\
\hline & Do. & 2014 & $\begin{array}{c}\text { Indirect- } \\
\text { discharge } \\
\text { measurement }\end{array}$ & 938 & 938 & 938 & 0 & 0 & $\mathrm{SAC}$ \\
\hline 07227300 & Sand Draw near Clayton, N. Mex. & 2014 & $\begin{array}{l}\text { Non-exceedance } \\
\text { bound }\end{array}$ & 12,200 & 8,730 & 42,100 & 500 & \pm 100 & $\mathrm{SAC}$ \\
\hline \multirow[t]{3}{*}{07232650} & Aqua Frio Creek near Felt, Okla. & 2014 & $\begin{array}{l}\text { Non-exceedance } \\
\text { bound }\end{array}$ & 1,900 & 1,900 & 1,900 & 38 & 0 & Gage record \\
\hline & Do. & 2014 & $\begin{array}{l}\text { Non-exceedance } \\
\text { bound }\end{array}$ & 6,540 & 5,600 & 11,200 & 2,000 & \pm 500 & $\begin{array}{l}\text { Step- } \\
\text { rackwater }\end{array}$ \\
\hline & & & & & & & Mean & 630 years & \\
\hline
\end{tabular}




\section{Appendix 3. Annual Exceedance-Probability Discharges}

Table 3-1. Summary of the annual exceedance-probability discharges at the 188 streamgages used in the regression analysis of natural streams in eastern Colorado, 2015.

[USGS, U.S. Geological Survey; $Q_{0.5}, Q_{0.2}, Q_{0.1}, Q_{0.04}, Q_{0.02}, Q_{0.01}, Q_{0.005}, Q_{0.002}$, discharge with an annual exceedance-probability of $0.5,0.2,0.1,0.04,0.02,0.01,0.005$, 0.002, respectively; Wyo., Wyoming; Nebr., Nebraska; Colo., Colorado; Kans., Kansas; Okla., Oklahoma; N. Mex., New Mexico; Tex., Texas; NA, not applicable]

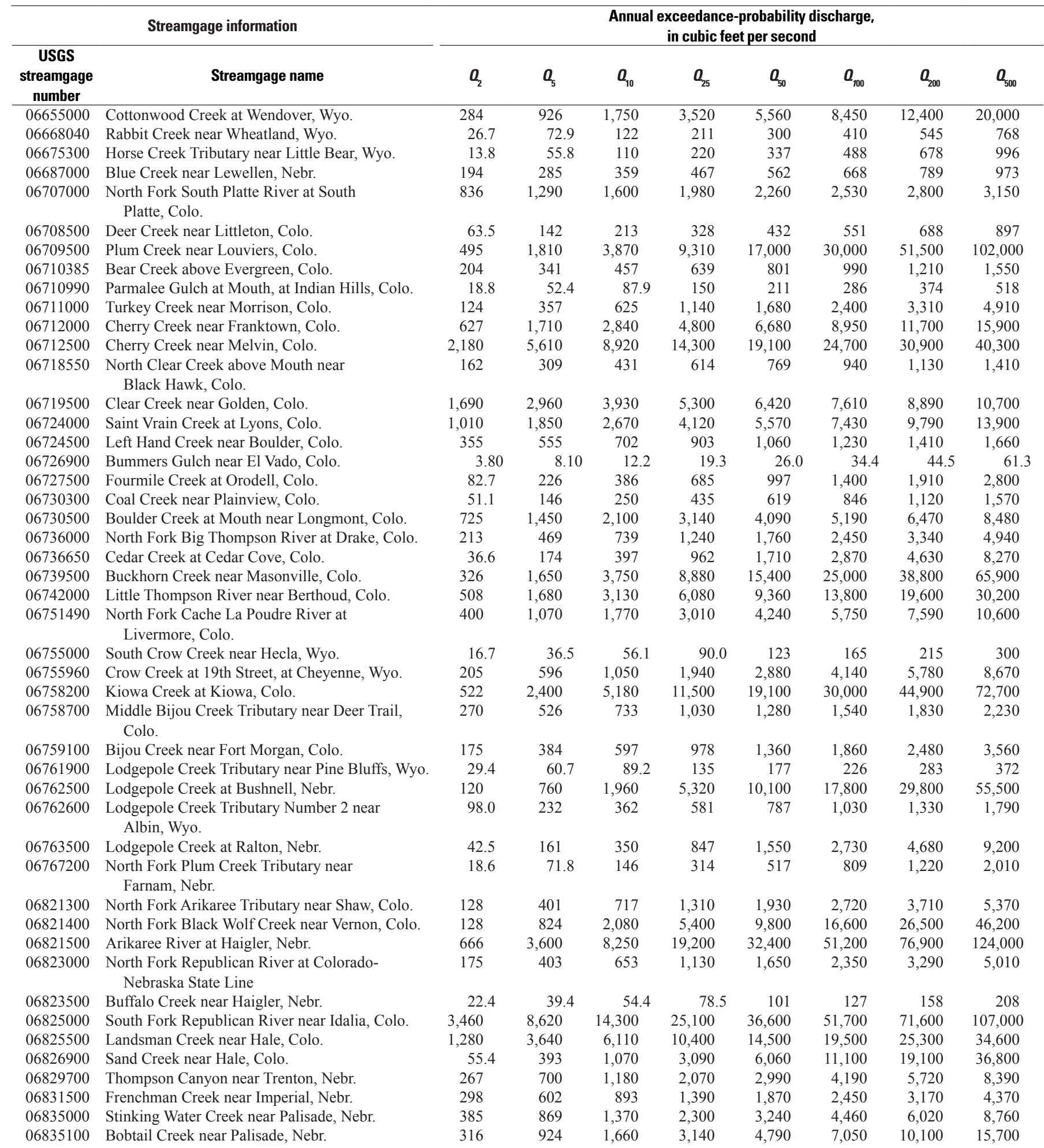


Table 3-1. Summary of the annual exceedance-probability discharges at the 188 streamgages used in the regression analysis of natural streams in eastern Colorado, 2015.-Continued

[USGS, U.S. Geological Survey; $Q_{0.5}, Q_{0.2}, Q_{0.1}, Q_{0.04}, Q_{0.02}, Q_{0.01}, Q_{0.005}, Q_{0.002}$, discharge with an annual exceedance-probability of 0.5, 0.2, 0.1, 0.04, 0.02, 0.01, 0.005, 0.002, respectively; Wyo., Wyoming; Nebr., Nebraska; Colo., Colorado; Kans., Kansas; Okla., Oklahoma; N. Mex., New Mexico; Tex., Texas; NA, not applicable]

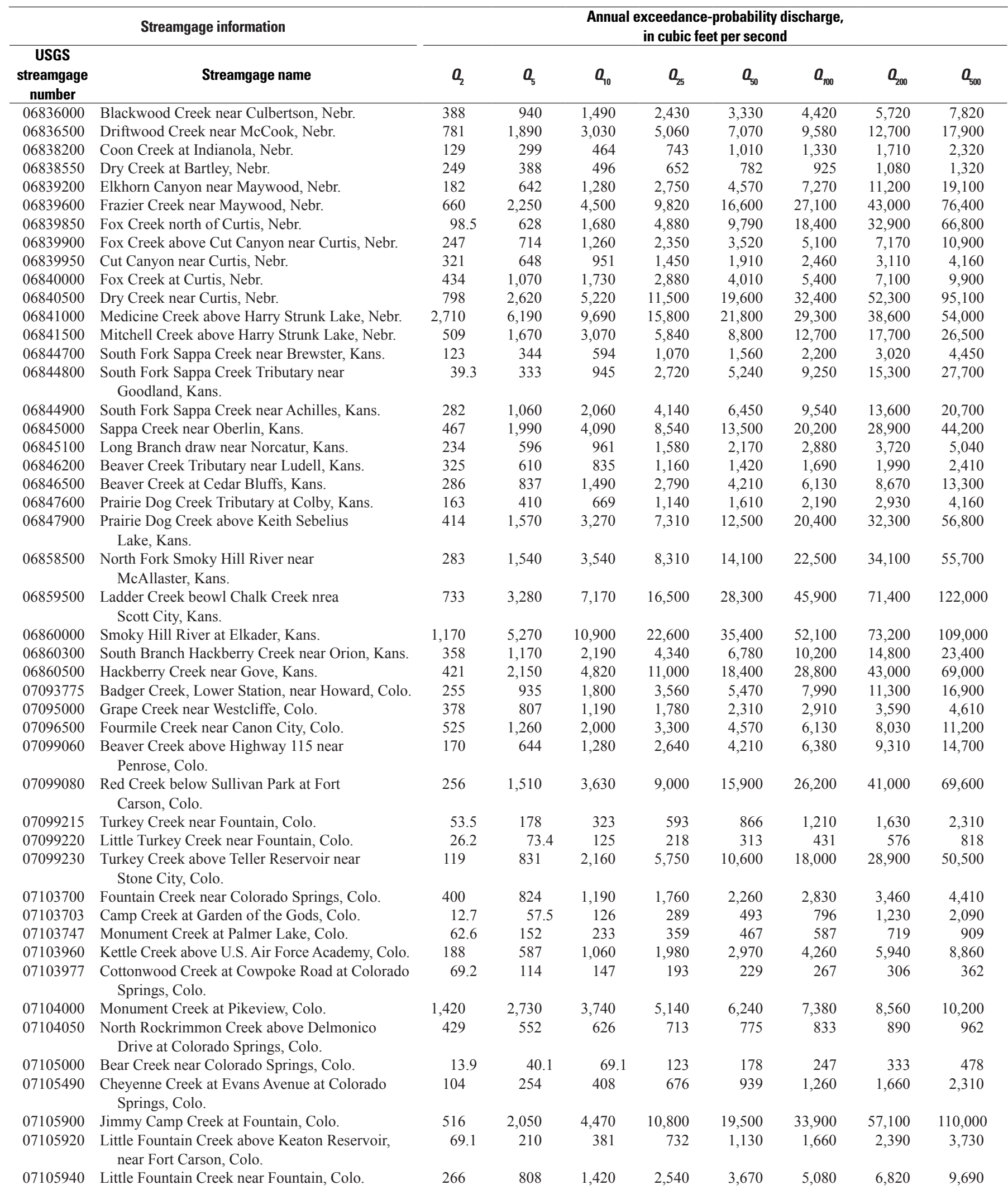


Table 3-1. Summary of the annual exceedance-probability discharges at the 188 streamgages used in the regression analysis of natural streams in eastern Colorado, 2015.-Continued

[USGS, U.S. Geological Survey; $Q_{0.5}, Q_{0.2}, Q_{0.1}, Q_{0.04}, Q_{0.02}, Q_{0.01}, Q_{0.005}, Q_{0.002}$, discharge with an annual exceedance-probability of 0.5, 0.2, 0.1, 0.04, 0.02, 0.01, 0.005, 0.002, respectively; Wyo., Wyoming; Nebr., Nebraska; Colo., Colorado; Kans., Kansas; Okla., Oklahoma; N. Mex., New Mexico; Tex., Texas; NA, not applicable]

\begin{tabular}{|c|c|c|c|c|c|c|c|c|c|}
\hline \multicolumn{2}{|r|}{ Streamgage information } & \multicolumn{8}{|c|}{$\begin{array}{l}\text { Annual exceedance-probability discharge, } \\
\text { in cubic feet per second }\end{array}$} \\
\hline 07106500 & Fountain Creek at Pueblo, Colo. & 4,630 & 10,000 & 15,200 & 23,900 & 32,200 & 42,300 & 54,400 & 73,900 \\
\hline 07107500 & St. Charles River at Burnt Mill, Colo. & 921 & 2,890 & 5,210 & 9,700 & 14,400 & 20,600 & 28,500 & 42,000 \\
\hline 07108900 & St. Charles River at Vineland, Colo. & 2,370 & 5,460 & 8,600 & 14,200 & 19,700 & 26,600 & 35,100 & 49,600 \\
\hline 07112500 & Huerfano River at Badito, Colo. & 963 & 2,180 & 3,350 & 5,300 & 7,130 & 9,300 & 11,900 & 16,000 \\
\hline 07114000 & $\begin{array}{l}\text { Cucharas River at Boyd Ranch near La Veta, } \\
\text { Colo. }\end{array}$ & 111 & 220 & 308 & 438 & 546 & 663 & 789 & 970 \\
\hline 07116000 & $\begin{array}{l}\text { Huerfano River below Huerfano Valley Dam near } \\
\text { Undercliffe, Colo. }\end{array}$ & 3,670 & 7,320 & 10,400 & 14,800 & 18,600 & 22,700 & 27,200 & 33,700 \\
\hline 07118000 & Apishapa River near Aguilar, Colo. & 2,430 & 3,750 & 4,670 & 5,840 & 6,730 & 7,620 & 8,520 & 9,730 \\
\hline 07124300 & Long Canyon Creek near Madrid, Colo. & 1,190 & 2,240 & 3,100 & 4,330 & 5,350 & 6,450 & 7,640 & 9,350 \\
\hline 07124500 & Purgatoire River at Trinidad, Colo. & 5,790 & 12,100 & 17,400 & 25,400 & 32,100 & 39,500 & 47,600 & 59,200 \\
\hline 07125100 & Frijole Creek near Alfalfa, Colo. & 1,640 & 3,770 & 5,840 & 9,300 & 12,600 & 16,500 & 21,100 & 28,400 \\
\hline 07125500 & San Francisco Creek near Alfalfa, Colo. & 2,530 & 5,010 & 7,090 & 10,200 & 12,800 & 15,700 & 18,900 & 23,500 \\
\hline 07126100 & Luning Arroyo near Model, Colo. & 580 & 1,730 & 3,140 & 6,030 & 9,290 & 13,800 & 19,900 & 31,400 \\
\hline 07126140 & Van Bremer Arroyo near Tyrone, Colo. & 43.3 & 186 & 379 & 783 & 1,230 & 1,820 & 2,570 & 3,880 \\
\hline 07126200 & Van Bremer Arroyo near Model, Colo. & 476 & 1,790 & 3,440 & 6,680 & 10,100 & 14,500 & 19,900 & 29,100 \\
\hline 07126325 & $\begin{array}{l}\text { Taylor Arroyo below Rock Crossing, near } \\
\text { Thatcher, Colo. }\end{array}$ & 188 & 705 & 1,360 & 2,690 & 4,110 & 5,980 & 8,360 & 12,400 \\
\hline 07126390 & Lockwood Canyon Creek near Thatcher, Colo. & 108 & 333 & 580 & 1,020 & 1,460 & 1,990 & 2,620 & 3,620 \\
\hline 07126415 & $\begin{array}{l}\text { Red Rock Canyon Creek at Mouth near } \\
\text { Thatcher, Colo. }\end{array}$ & 407 & 1,010 & 1,620 & 2,660 & 3,660 & 4,870 & 6,330 & 8,670 \\
\hline 07126470 & Chacuaco Creek at Mouth near Timpas, Colo. & 1,790 & 4,650 & 7,650 & 13,000 & 18,300 & 24,900 & 32,900 & 46,300 \\
\hline 07133200 & Clay Creek Tributary near Deora, Colo. & 33.5 & 711 & 3,170 & 14,400 & 36,900 & 83,600 & 173,000 & 405,000 \\
\hline 07134100 & Big Sandy Creek near Lamar, Colo. & 235 & 623 & 1,030 & 1,770 & 2,500 & 3,410 & 4,530 & 6,380 \\
\hline 07134990 & Wild Horse Creek above Holly, Colo. & 193 & 446 & 697 & 1,130 & 1,550 & 2,070 & 2,700 & 3,740 \\
\hline 07135000 & Two Butte Creek near Holly, Colo. & 101 & 464 & 982 & 2,100 & 3,380 & 5,100 & 7,350 & 11,300 \\
\hline 07138600 & $\begin{array}{l}\text { White Woman Creek Tributary near Selkirk, } \\
\text { Kans. }\end{array}$ & 62.3 & 159 & 257 & 427 & 590 & 788 & 1,030 & 1,410 \\
\hline 07138650 & White Woman Creek near Leoti, Kans. & 203 & 744 & 1,420 & 2,760 & 4,180 & 6,030 & 8,360 & 12,300 \\
\hline 07138800 & Lion Creek Tributary near Modoc, Kans. & 134 & 168 & 189 & 213 & 229 & 245 & 260 & 279 \\
\hline 07140600 & Pawnee River Tributary near Kalvesta, Kans. & 234 & 710 & 1,280 & 2,440 & 3,710 & 5,440 & 7,740 & 11,900 \\
\hline 07141400 & $\begin{array}{l}\text { South Fork Walnut Creek Tributary near } \\
\text { Dighton, Kans. }\end{array}$ & 66.6 & 99.4 & 123 & 154 & 179 & 204 & 231 & 268 \\
\hline 07153500 & Dry Cimarron River near Guy, N. Mex. & 2,800 & 6,680 & 10,800 & 18,200 & 25,900 & 35,700 & 48,200 & 69,900 \\
\hline 07154400 & Carrizozo Creek near Kenton, Okla. & 1,590 & 4,090 & 6,570 & 10,700 & 14,600 & 19,100 & 24,400 & 32,700 \\
\hline 07154500 & Cimarron River near Kenton, Okla. & 3,650 & 10,000 & 16,600 & 28,200 & 39,300 & 52,700 & 68,700 & 94,200 \\
\hline 07154650 & Tesesquite Creek near Kenton, Okla. & 1,220 & 3,920 & 6,950 & 12,400 & 17,800 & 24,400 & 32,200 & 44,700 \\
\hline 07155100 & Cold Springs Creek near Wheeless, Okla. & 55.2 & 282 & 632 & 1,450 & 2,440 & 3,840 & 5,760 & 9,320 \\
\hline 07155590 & Cimarron River near Elkhart, Kans. & 131 & 2,140 & 8,120 & 30,600 & 68,700 & 138,000 & 253,000 & 511,000 \\
\hline 07155900 & $\begin{array}{l}\text { North Fork Cimarron River Tributary near } \\
\text { Elkhart, Kans. }\end{array}$ & 34.5 & 736 & 3,200 & 14,000 & 34,600 & 75,400 & 150,000 & 333,000 \\
\hline 07156000 & $\begin{array}{l}\text { North Fork Cimarron River Tributary near } \\
\text { Richfield, Kans. }\end{array}$ & 709 & 2,460 & 4,570 & 8,640 & 12,900 & 18,300 & 25,100 & 36,400 \\
\hline 07156010 & North Fork Cimarron River at Richfield, Kans. & 763 & 3,530 & 8,030 & 19,500 & 35,000 & 59,500 & 97,000 & 177,000 \\
\hline 07156100 & Sand Arroyo Creek near Johnson, Kans. & 70.8 & 247 & 458 & 864 & 1,290 & 1,820 & 2,480 & 3,580 \\
\hline 07156220 & Bear Creek near Johnson, Kans. & 927 & 2,580 & 4,350 & 7,470 & 10,500 & 14,300 & 18,800 & 26,200 \\
\hline 07156600 & Cimarron River Tributary near Moscow, Kans. & 636 & 1,270 & 1,790 & 2,560 & 3,210 & 3,910 & 4,670 & 5,780 \\
\hline
\end{tabular}


Table 3-1. Summary of the annual exceedance-probability discharges at the 188 streamgages used in the regression analysis of natural streams in eastern Colorado, 2015.-Continued

[USGS, U.S. Geological Survey; $Q_{0.5}, Q_{0.2}, Q_{0.1}, Q_{0.04}, Q_{0.02}, Q_{0.01}, Q_{0.005}, Q_{0.002}$, discharge with an annual exceedance-probability of 0.5, 0.2, 0.1, 0.04, 0.02, 0.01, 0.005, 0.002, respectively; Wyo., Wyoming; Nebr., Nebraska; Colo., Colorado; Kans., Kansas; Okla., Oklahoma; N. Mex., New Mexico; Tex., Texas; NA, not applicable]

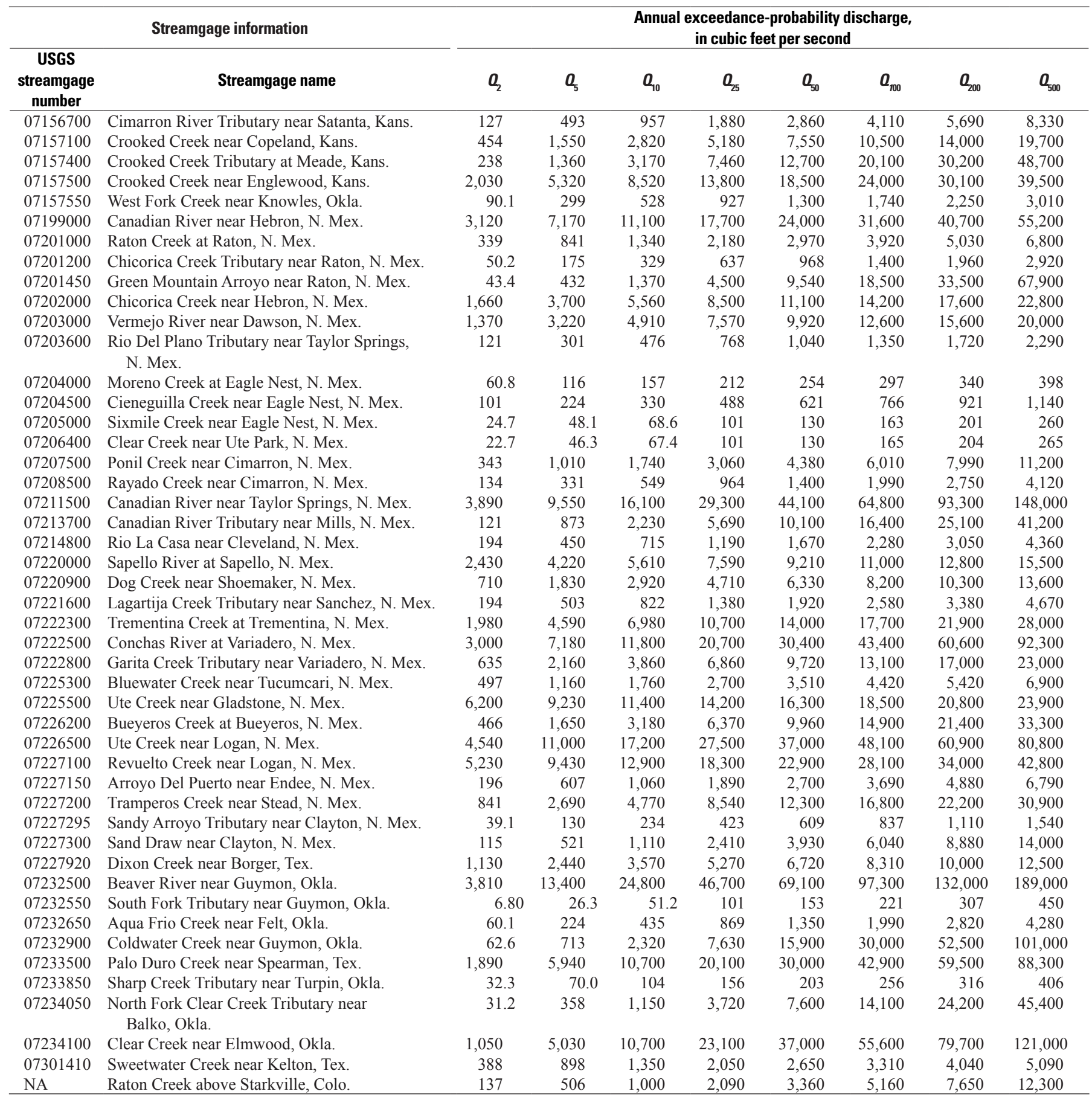




\section{Appendixes 4-6}

[available at http://dx.doi.org/10.3133/sir20165099]

Appendix 4. Flood-frequency analysis at the 188 streamgages included in the report

Appendix 5. Variance of prediction values and annual exceedance-probability discharges for streamgages in the foothills and plains hydrologic regions of Colorado and selected streamgages in adjacent states

Appendix 6. Final peak-streamflow reqional-regression equation analyses in the foothills and plains hydrologic regions of Colorado 
Publishing support provided by the Denver Publishing Service Center

For more information concerning this publication, contact: Director, USGS Colorado Water Science Center

Box 25046, Mail Stop 415

Denver, CO 80225

(303) 236-4882

Or visit the Colorado Water Science Center Web site at http://co.water.usgs.gov/

This publication is available online at http://dx.doi.org/10.3133/sir20165099 

